\title{
AN ANALYSIS OF ELIMINATING ELECTROOSMOTIC FLOW IN A MICROFLUIDIC PDMS CHIP
}

\author{
A Thesis \\ presented to \\ the Faculty of California Polytechnic State University, \\ San Luis Obispo
}

\author{
In Partial Fulfillment \\ of the Requirements for the Degree \\ Master of Science in Biomedical Engineering
}

by

Cécile Deirdre Redington

August 2013 
(C) 2013

Cécile Deirdre Redington

ALL RIGHTS RESERVED

Page ii 


\section{COMMITTEE MEMBERSHIP}

TITLE: An Analysis Of Eliminating Electroosmotic Flow In $\mathrm{A}$ Microfluidic PDMS Chip

AUTHOR: $\quad$ Cécile Deirdre Redington

DATE SUBMITTED: August 2013

COMMITTEE CHAIR: David Clague, PhD

Associate Professor of Biomedical and General Engineering

COMMITTEE MEMBER: Robert Crockett, PhD

Director of General Engineering

COMMITTEE MEMBER: Richard Savage, PhD

Associate Professor of Biomedical and General

Engineering 


\section{ABSTRACT \\ An Analysis Of Eliminating Electroosmotic Flow In A Microfluidic PDMS Chip \\ Cécile Deirdre Redington}

The goal of this project is to eliminate electroosmotic flow (EOF) in a microfluidic chip. EOF is a naturally occurring phenomenon at the fluid-surface interface in microfluidic chips when an electric field is applied across the fluid. When isoelectric focusing (IEF) is carried out to separate proteins based on their surface charge, the analytes must remain in the separation chamber, and not migrate to adjacent features in the microfluidic chip, which happens with EOF.

For this project, a microfluidic chip was designed and commissioned to be photolithographically transferred onto a Si wafer. A PDMS component was then casted on the Si wafer and plasma bonded to a glass substrate. This chip was initially designed to carry out continuous IEF, and the focus of the project was shifted to the analysis of eliminating EOF in a microfluidic chamber.

Per previous research test methods, methylcellulose will be used to analyze the

phenomenon of electroosmotic flow in the chamber. A COMSOL model is used a theoretical basis of comparison when analyzing the flow velocities of the treated versus untreated microfluidic chips.

The purpose of this project is to use the research performed in on this chip as a precursor to future analyses of continuous IEF on microfluidic chips in the Cal Poly Microfluidics group.

Page iv 


\section{ACKNOWLEDGMENTS}

I am deeply grateful of the support and guidance Dr. David Clague provided throughout the project. His significant role in Biomedical Engineering Department has opened up new opportunities for microfluidic investigations, and this project would not have been possible without him.

I would also like to thank Dr. Crockett and Dr. Savage for their assistance on the defense committee, and for being available during the entire defense process. I greatly appreciate the guidance Dr. Savage offered during the microfluidic chip fabrication, and for giving the Cal Poly Microfluidics group access to the Materials Engineering clean room and instruction on the use of its equipment.

I would also like to thank Josh Fadriquela for his assistance during the initial phases of the project, and the Microfluidics group for their insight and collaboration throughout project. I would also like to acknowledge the Stanford Microfluidics Foundry for aiding in multilayer PDMS chip design, and California Fine Wire for donating samples to the group.

I would like to thank my parental units for their unconditional love and guidance; my favorite sister for being the strongest and most loving person. Thanks to my grandparents for raising me, and teaching me the love of learning. I would like to also thank my best friend, Max, for the adventures, unrelenting support, and for bringing the sun every day. 


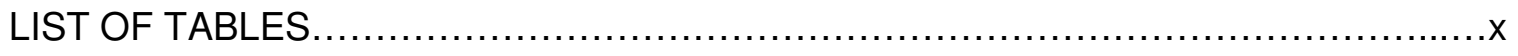

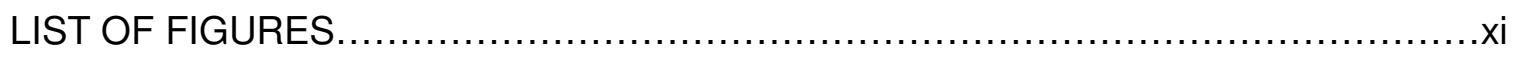

CHAPTER

$1 \quad$ INTRODUCTION

1.1 The Importance of Microscale Total Analysis Systems ..................................

1.1.1 Application: Bacterial Detection and Global Health ............................

1.1.2 Application: Military Applications .....................................................

1.1.3 Application: Cancer Detection ....................................................

1.1.4 Application: Proteome Analysis....................................................

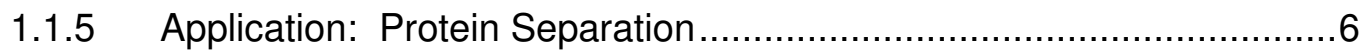

1.2 Chip considerations based on prior research projects...................................

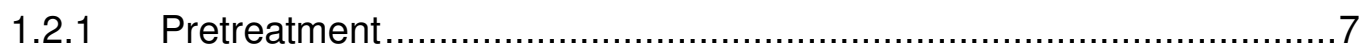

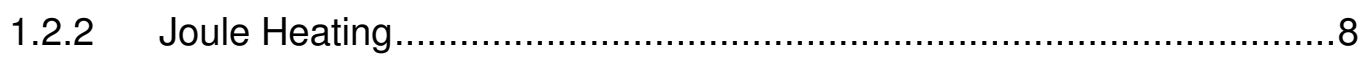

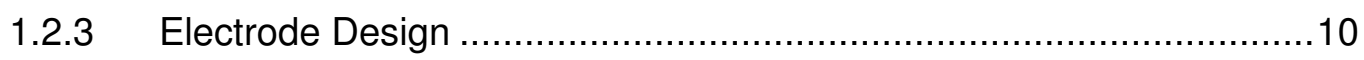

1.2.4 Hydrophilicity and Hydrophobicity of PDMS ……...........................10

1.2.5 Restricting flow between Chambers...............................................13

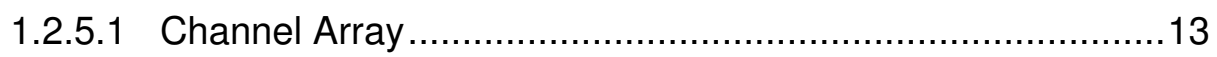

1.2.5.2 Gel Barrier ............................................................... 14

1.2.5.3 Salt Bridge Barrier.......................................................... 15

1.2.5.4 Using Channel Depth ......................................................15

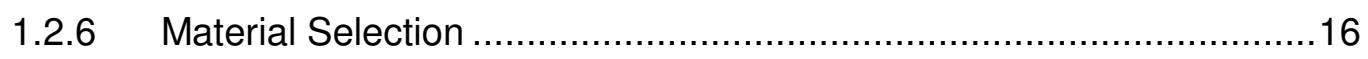

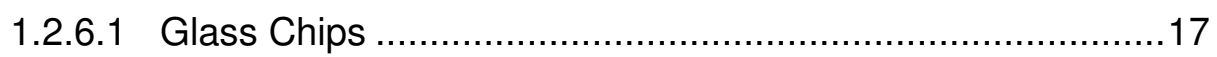

1.2.6.2 Polydimethylsiloxane Chips .............................................17

1.2.7 The pH Gradient ................................................................. 18

1.2.7.1 pH Gradient through Electrolysis Products..........................18

1.2.7.2 pH Gradient through Ampholytes ......................................19

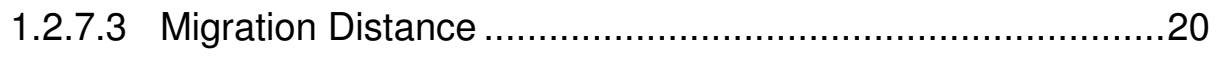

1.2.7.4 The Importance of Eliminating Electroosmosis in $\mu$ TAS .......21

1.2.8 Traditional 2-Dimensional Electrophoresis .......................................21 


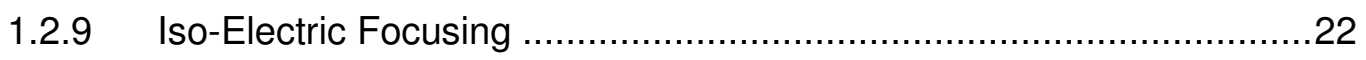

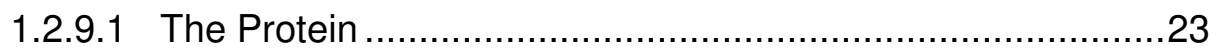

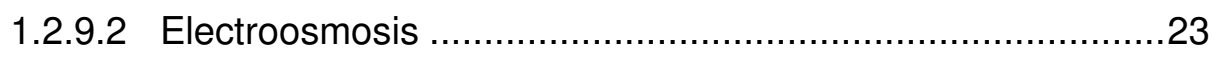

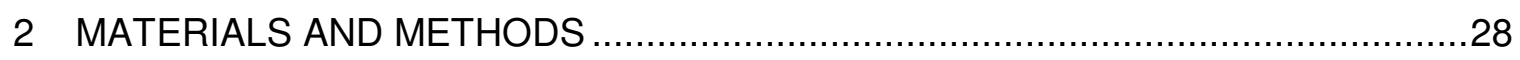

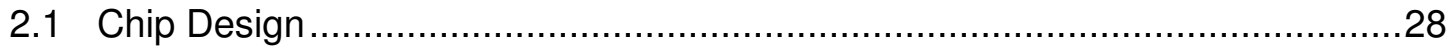

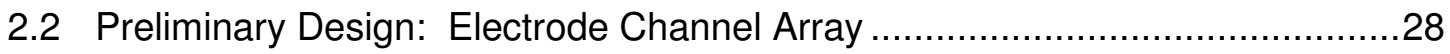

2.3 Preliminary Design: Separate Buffer Inlets ................................................31

2.4 Preliminary Design: Channel Array Design .....................................................35

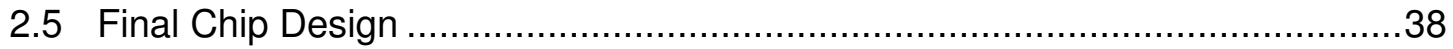

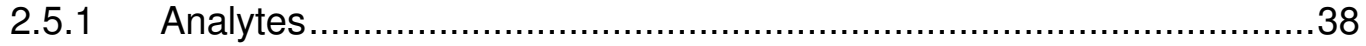

2.5.2 Inlet and Outlet Placement................................................................39

2.5.3 Electrode Chamber Design ...................................................... 40

2.5.4 Membrane Channel Design ......................................................... 41

2.5.5 Separation Chamber Design ...................................................... 44

2.5.6 PDMS Master Mold............................................................ 44

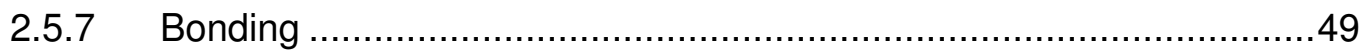

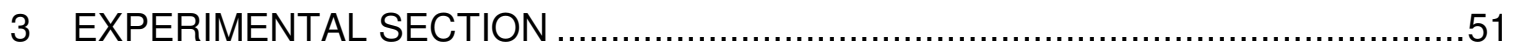

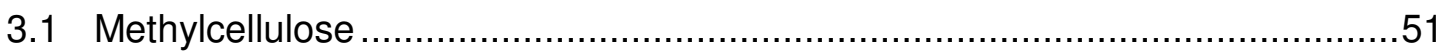

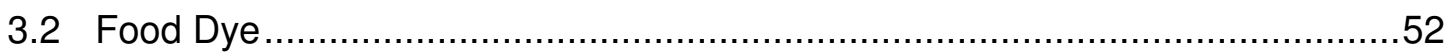

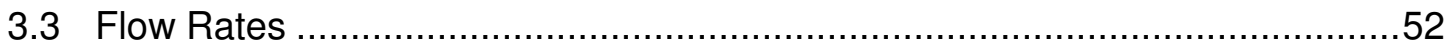

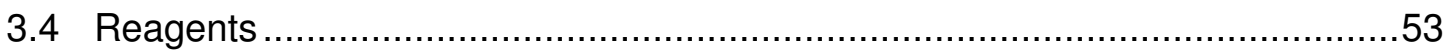

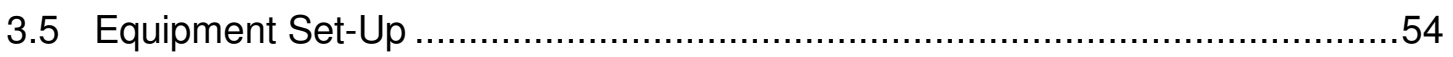

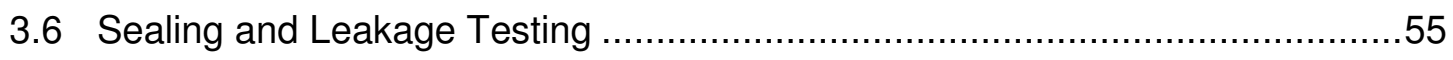

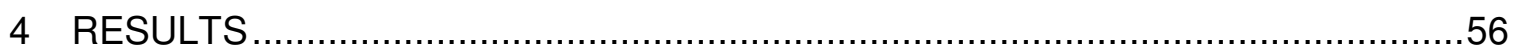

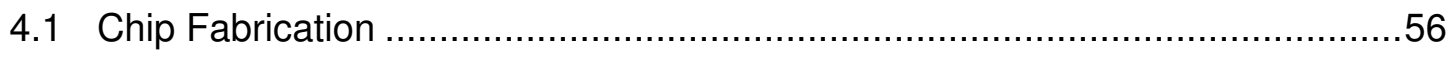

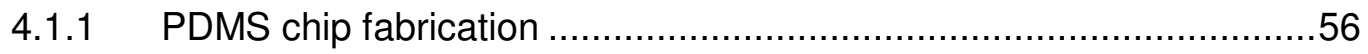

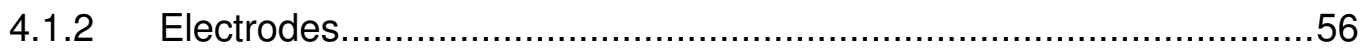

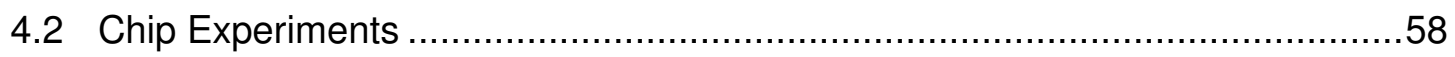

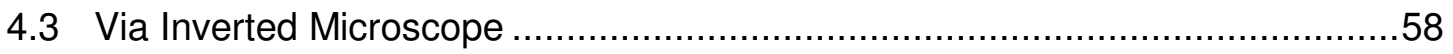

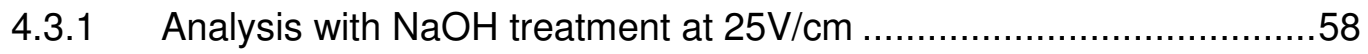

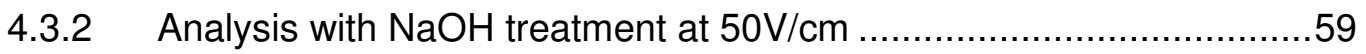

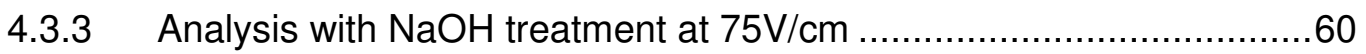

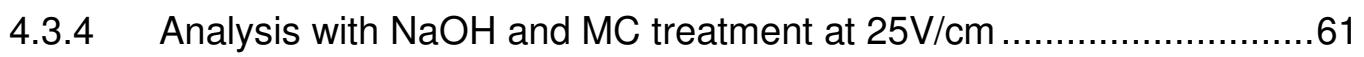

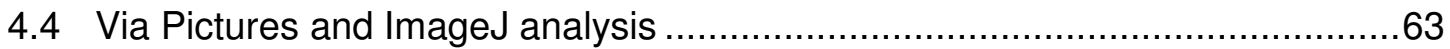

Page vii 


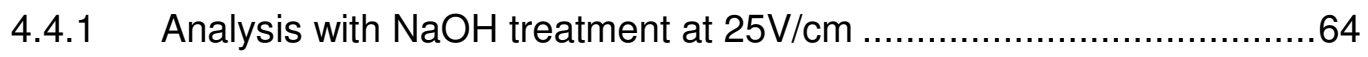

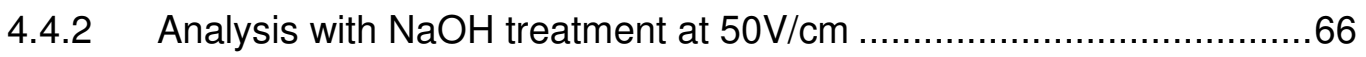

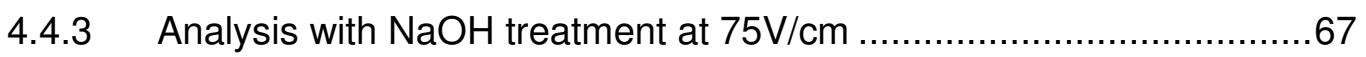

4.4.4 Analysis with $\mathrm{NaOH}$ and $\mathrm{MC}$ treatment at $25 \mathrm{~V} / \mathrm{cm} \ldots \ldots \ldots \ldots \ldots \ldots \ldots . . . \ldots . \ldots . \ldots \ldots$

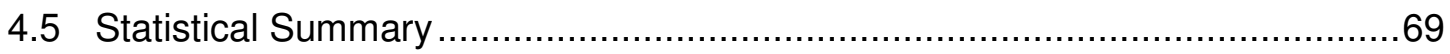

4.5.1 Statistical Analysis of Inverted Microscope results ...........................69

4.5.2 Statistical Analysis of Pictures and ImageJ results..........................71

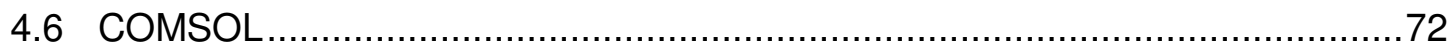

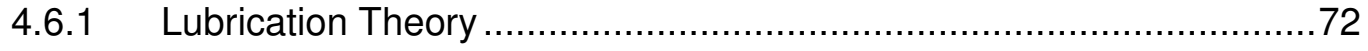

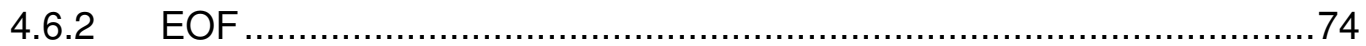

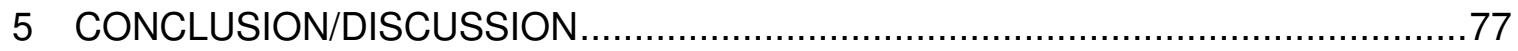

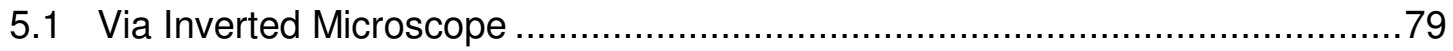

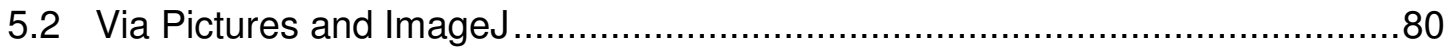

5.3 Flow Velocity comparison with COMSOL results ...................................... 80

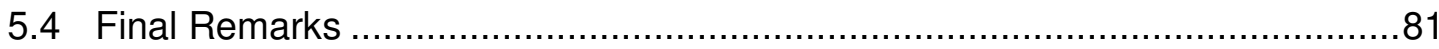

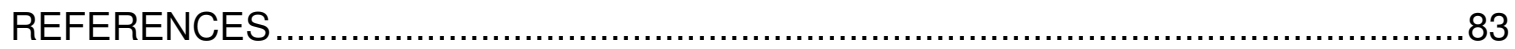

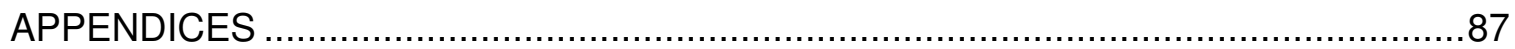

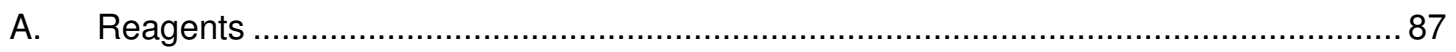

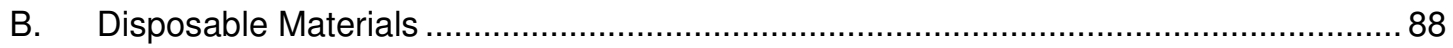

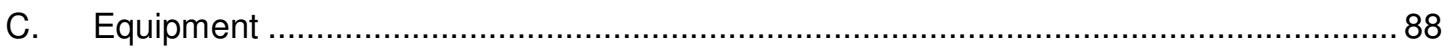

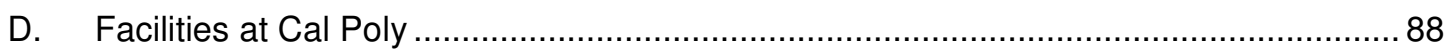

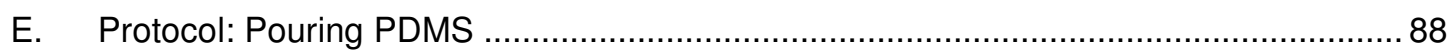

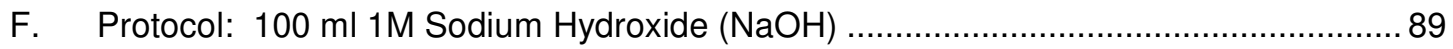

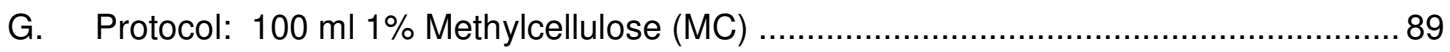

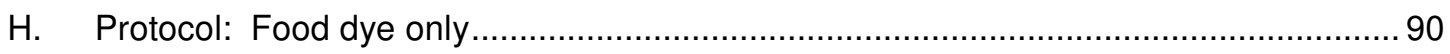

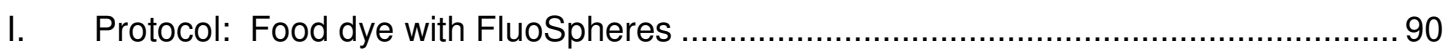

J. Protocol for Test 1: Preliminary Testing to analyze EOF without treatment at

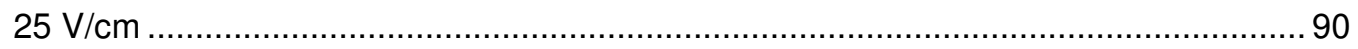

K. Protocol for Test 2: Analysis with $\mathrm{NaOH}$ treatment at $25 \mathrm{~V} / \mathrm{cm}, 50 \mathrm{~V} / \mathrm{cm}$, and $75 \mathrm{~V} / \mathrm{cm}$

L. Protocol for Test 3: Analysis with $\mathrm{NaOH}$ and $\mathrm{MC}$ surface treatments at 25

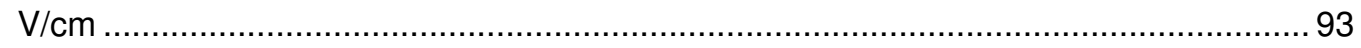

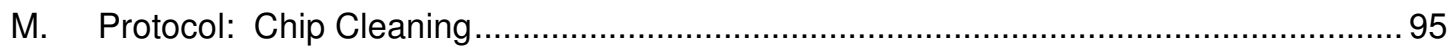

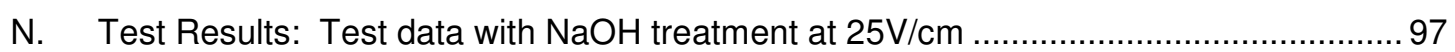

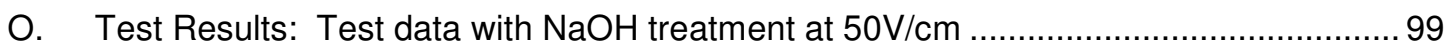

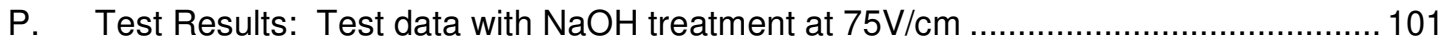


Q. Test Results: Test data with $\mathrm{NaOH}$ and $\mathrm{MC}$ treatment at $25 \mathrm{~V} / \mathrm{cm} \ldots \ldots \ldots \ldots \ldots \ldots \ldots \ldots . . . . . . . . . . . . .103$

\section{Page ix}




\section{LIST OF TABLES}

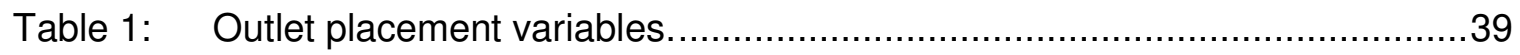

Table 2: Post geometry specifications........................................................... 41

Table 3: $\quad$ Summary of test results per the Inverted Microscope .................................69

Table 4: $\quad$ Summary of test results per Pictures and Image

Table 5: $\quad$ COMSOL Parameters to analyze EOF...................................................74 


\section{LIST OF FIGURES}

Figure 1: Ion constitution at fluid/folid interface (Breuer 2005) ................................24

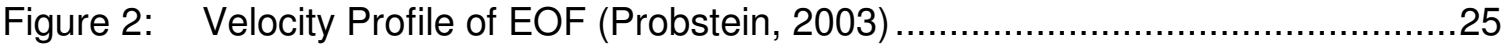

Figure 3: Channel Array Design- Schematic .......................................................29

Figure 4: Channel Array Design- COMSOL modeling of overall view (above) and detailed view (below) of the electric potential across the chip......................30

Figure 5: Channel Array Design- Voltage drop ..........................................................31

Figure 6: Design 2- Track etched membrane, separate $\mathrm{pH}$ buffer inlets, and multiple sample collection weirs.

Figure 7: Separate Buffer Inlet Design- COMSOL modeling of $\mathrm{pH}$ level

Figure 8: Separate Buffer Inlet Design- $\mathrm{H}^{+}$Concentration along the length of the chip

Figure 9: Separate Buffer Inlet Design- $\mathrm{H}^{+}$Concentration across the chip at $0.0005 \mathrm{~m}$ from inlet

Figure 10: Separate Buffer Inlet Design- $\mathrm{H}^{+}$Concentration gradient across the width of the channel at $0.01 \mathrm{~cm}$ after the inlets.

Figure 11: Channel Array Design- Schematic ...........................................................

Figure 12: Channel Array Design- COMSOL modeling of $\mathrm{pH}$ concentrations.....

Figure 13: Channel Array Design- $\mathrm{H}^{+}$Concentration immediately after the staggered blocks

Figure 14: Microfluidic chamber post designs

Figure 15: COMSOL modeling of velocity profile of electrode and separation chamber in overall view (above) and detailed view (below).

Figure 16: Velocity profile through height of the electrode chamber

Figure 17: Velocity profile through height of the membrane chamber..

Figure 18: Placement of chip designs on wafer..................................................46

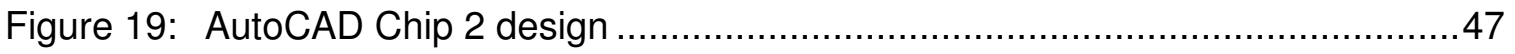

Figure 20: AutoCAD Chip 1 design ...................................................................

Figure 21: Cross-sectional view of Layer organization in AutoCAD ............................49

Figure 22: Oxygen plasma treatment of PDMS (Bodas and Khan Malek, 2006) ..........50

Figure 23: PDMS with copper electrodes bonded to glass slide .................................50

Figure 24: Overall Experimental Set-up. ……………...........................................54 


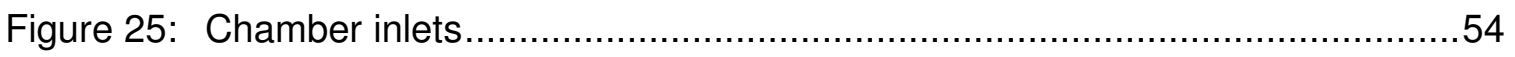

Figure 26: Electrode "sewing" with 40 gauge wire................................................57

Figure 27: StatGraphics results of testing with $\mathrm{NaOH}$ treatment at $25 \mathrm{~V} / \mathrm{cm} \ldots \ldots \ldots \ldots \ldots . . . . . . .59$

Figure 28: StatGraphics results of testing with $\mathrm{NaOH}$ treatment at $50 \mathrm{~V} / \mathrm{cm} \ldots \ldots \ldots \ldots \ldots . . . . . . .60$

Figure 29: StatGraphics results of testing with $\mathrm{NaOH}$ treatment at $75 \mathrm{~V} / \mathrm{cm} \ldots \ldots \ldots \ldots \ldots . \ldots 1$

Figure 30: StatGraphics results of testing with $\mathrm{MC}$ treatment at $25 \mathrm{~V} / \mathrm{cm} \ldots \ldots \ldots \ldots \ldots \ldots . \ldots \ldots$

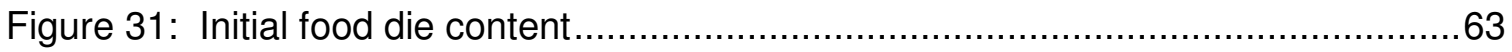

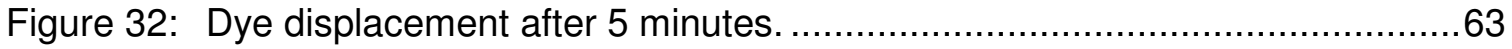

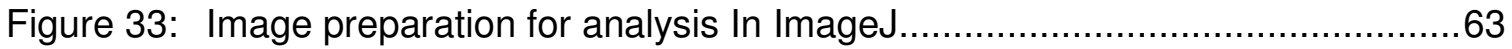

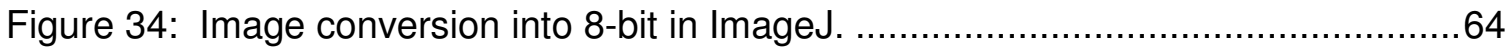

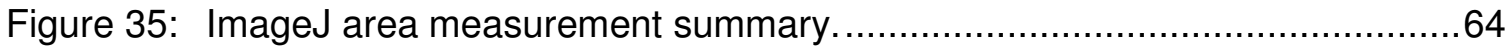

Figure 36: StatGraphics results of testing with $\mathrm{NaOH}$ treatment at $25 \mathrm{~V} / \mathrm{cm} \ldots \ldots \ldots \ldots \ldots . \ldots 5$

Figure 37: StatGraphics results of testing with $\mathrm{NaOH}$ treatment at $50 \mathrm{~V} / \mathrm{cm} \ldots \ldots \ldots \ldots \ldots . \ldots 6$

Figure 38: StatGraphics results of testing with $\mathrm{NaOH}$ treatment at $75 \mathrm{~V} / \mathrm{cm} \ldots \ldots \ldots \ldots \ldots . \ldots 7$

Figure 39: StatGraphics results of testing with $\mathrm{NaOH}$ and $\mathrm{MC}$ treatment at $25 \mathrm{~V} / \mathrm{cm} \ldots . .68$

Figure 40: ANOVA Inverted Microscope results of $\mathrm{NaOH}$ (at 25, 50, and 75V/cm) and $\mathrm{NaOH}+\mathrm{MC}($ at $25 \mathrm{~V} / \mathrm{cm}$ ) treatments. ........................................ 70

Figure 41: Statgraphics analysis per Inverted Microscope- Results of $\mathrm{NaOH}$ only

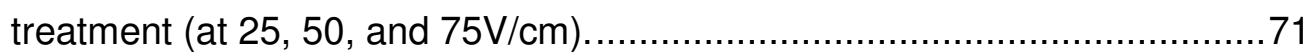

Figure 42: ANOVA ImageJ results of $\mathrm{NaOH}$ (at 25,50 , and $75 \mathrm{~V} / \mathrm{cm}$ ) and $\mathrm{NaOH}+\mathrm{MC}$ (at $25 \mathrm{~V} / \mathrm{cm}$ ) treatments.............................................................. 72

Figure 43: COMSOL 3D model for lubrication theory analysis. ..............................72

Figure 44: Cross-sectional slice of flow velocity ................................................ 73

Figure 45: Flow velocity in the electrode (left) and membrane (right) chambers..........73

Figure 46: Cross-section of the electrode chambers and the separation chamber .......75

Figure 47: Magnified cross-section of the left electrode chamber and separation chamber showing the velocity profile.................................................75

Figure 48: Vertical velocity profile of the separation chamber at $1.4 \times 10^{-4} \mathrm{~m} \mathrm{\ldots ..........76}$

Figure 49: Chip without any treatment shortly after application of $25 \mathrm{~V} / \mathrm{cm}$. ................77

Figure 50: Formation of bubbles at the Cathode(left) and particles at the Anode

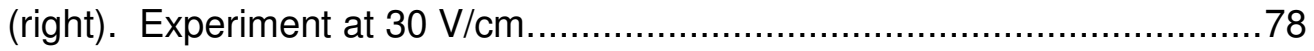

Figure 51: Lack of bubbles and particles at the electrodes (Cathode (left) and Anode (right)) when experiment is carried out at $25 \mathrm{~V} / \mathrm{cm}$. 
Figure 52: Box-and-Whisker plot of Inverted Microscope trials of $\mathrm{NaOH}$ treatment at $25 \mathrm{~V} / \mathrm{cm}$

Figure 53: Data summary of all test runs with $\mathrm{NaOH}$ treatment at $25 \mathrm{~V} / \mathrm{cm}$

Figure 54: Box-and-Whisker plot of Inverted Microscope trials of $\mathrm{NaOH}$ treatment at $50 \mathrm{~V} / \mathrm{cm}$

Figure 55: Data summary of all test runs with $\mathrm{NaOH}$ treatment at $50 \mathrm{~V} / \mathrm{cm}$ 100

Figure 56: Box-and-Whisker plot of Inverted Microscope trials of $\mathrm{NaOH}$ treatment at $75 \mathrm{~V} / \mathrm{cm}$ 101

Figure 57: Data summary of all test runs with $\mathrm{NaOH}$ treatment at $75 \mathrm{~V} / \mathrm{cm}$ 102

Figure 58: Box-and-Whisker plot of Inverted Microscope trials of $\mathrm{NaOH}$ and $\mathrm{MC}$ treatment at $25 \mathrm{~V} / \mathrm{cm}$. 103

Figure 59: Data summary of all test runs with $\mathrm{NaOH}$ and $\mathrm{MC}$ treatment at $25 \mathrm{~V} / \mathrm{cm} \ldots .103$ 


\section{INTRODUCTION}

\subsection{The Importance of Microscale Total Analysis Systems}

Microscale Total Analysis Systems ( $\mu$ TAS) are devices designed to accomplish tasks that conventionally involve much larger apparatuses and complex procedures performed in laboratories. These devices can be used for multiple applications, and mainly for disease detection or the classification of proteins and other biologics or chemicals in the medical field. Several applications of the $\mu$ TAS systems are in protein separation, biological and chemical weapon warfare, bacterial detection and global health, and research in the human genome.

\subsubsection{Application: Bacterial Detection and Global Health}

$\mu$ TAS, also known as Lab-on-a-chip (LOC) technologies have become attractive to physicians treating patients in developing countries, oftentimes located in remote locations (Chin, Linder et al. 2007). When working in geographically difficult to reach areas, physicians are concerned with the ease of transport of medical equipment. Physicians are mainly interested in this technology because of the small sample size and rapid analysis. Since only small samples and devices are used in LOCs, this method of detection is relatively inexpensive and efficient. This technology can aid in targeting global health issues, which also is closely linked to the macroeconomic health of a nation. Especially in third world countries, it is necessary to develop point-of-care (POC) systems that are inexpensive and can work without having a basic laboratory equipped with power and running water. Since developing countries are among the poorest of the world, they often depend on aide from western countries, and rely strongly on minimizing costs to maximize their resources. In these situations, it is necessary to develop technologies that are cost-effective. Physicians working in these conditions 
often do not have access to facilities to clean their equipment, and are in need of technology that is disposable.

Disease detection lends its importance to the treatment of conditions or at worst to a palliative treatment of patients not having access to full laboratories (Chin, Linder et al. 2007). Tropical illnesses such as Chagas disease, lymphatic filiarisis, dengue, etc., cause 500,000 deaths per year (Chin, Linder et al. 2007), of which $90 \%$ are concentrated in the sub-Saharan areas of Africa, which are amongst the most povertystricken countries of the world, and lacking the necessary facilities for health care. Many philanthropic foundations, such as the Doris Duke, Soros, and Gates, are funding research in this field because it shifts the need of performing procedures that are usually completed in a centralized lab to performing these procedures on a hand-held microfluidic chip. These point-of-care devices can help prevent the rapid spreading of epidemiological surveillance of diseases (Chin, Linder et al. 2007).

Conventional Polymerase Chain Reaction (PCR) devices for the amplification of nucleic acid molecules are based on accurate temperature control and enzyme reaction (Shen, Chen et al. 2005). This technology is used to generate several orders of magnitude of a portion of DNA by only using a few copies of the particular DNA sequence. The three main steps are the denaturing process that is carried out at 92-96 $\stackrel{\circ}{ } \mathrm{C}$, the annealing step at $37-65^{\circ} \mathrm{C}$, and finally the extension step at approximately $72^{\circ} \mathrm{C}$. During the denaturing process, the high temperature causes the sample doublestranded DNA to separate the strands from each other, forming single-stranded samples. The samples are then annealed, during which the primer, containing the targeted sequence binds to the complimentary sequence on the single-stranded DNA chain. During the extension phase, Taq polymerase synthesizes two new strands based on the template of the primer. This process of denaturing and synthesizing new DNA 
strands can then be repeated multiple times to create numerous copies of the original DNA segment. This technology is used to detect bacteria or viruses (e.g. AIDS) and to diagnose genetic disorders.

To circumvent the need for the complex thermal cycling, recent research has taken advantage of enzymes to perform the strand separation, known as Isothermal PCR. More specifically, Loop Mediated Isothermal Amplification (LAMP) uses Bst polymerase to separate the non-template from the template DNA strand without the need for the initial heating step (Chang, Chen et al. 2012). Also, the amount of Magnesium pyrophosphate increases as the product is amplified, and the color change can be easily seen with the addition of a SYBR green dye. Since this isothermal process carried out at $60-65^{\circ} \mathrm{C}$ and only requires a single temperature to carry out the amplification of a DNA sequence, it would eliminate the need for thermocycling devices, and thus make it a more practical option for diagnostic procedures in remote locations.

\subsubsection{Application: Military Applications}

With the evolution of biological warfare, the government has increasingly become interested in the detection of chemical and biological weapons. It is important to rapidly isolate and identify bacteria in medical diagnosis and biological warfare agent detection (Cabrera and Yager 2001). Research in this area is still in its basic developing phase, and is being continuously improved for rapid identification of toxins in biological warfare with the goal to create a system that can facilitate and decrease the time of detection.

In the following study, two biological toxins are identified using a combination of electrophoresis and immunoassay techniques. Researchers have used a microchip assay to identify staphylococcal enterotoxin B and fluorescein labeled cholera toxin B (Ewalt, Haigis et al. 2001). The design of such a chip was composed of a circular ring of point cathodes was laid out around a $5 \times 5$ micro assay, representing the anodes. 
Antibodies were immobilized at specific assay sites and the analyte -composed of a combination of the two toxins- was then placed on this chip. By applying electrophoresis (see Section 1.2.8) the proteins were driven towards the center of the circular ring of cathodes until it the protein reached its respective antibody assay site. The proteins were then detected using fluorescence from the top surface of the chip (Ewalt, Haigis et al. 2001). This chip demonstrated that it was possible to detect biological toxins within 6 minutes, and by only using a $10 \mu$ sample.

By further developing this technology, it can be placed in the field, and easily and effectively detect biological toxins in warfare.

\subsubsection{Application: Cancer Detection}

Since the 1960s, Free Flow Electrophoresis (FFE) has been used to separate different biological compounds for detection and collection (Frost and Bowser 2010). With the aid of FFE, scientists and researchers hope to detect biomarkers indicating diseases such as cancer or an individual's exposure to certain chemicals, as used in warfare. This technology could be implemented in the early detection of melanosome biogenesis after harvesting a patient's sample (Kushimoto, Basrur et al. 2001). To isolate the early stages of melanosome biogenesis, Kushimoto et al. used free-flow electrophoresis (FFE). The study successfully isolated lysosomes, endosomes, and other membrane-bound vesicles. This allowed for the group to study enzyme activity, morphology, cytochemistry, and many more aspects of the biogenesis (Kushimoto, Basrur et al. 2001).

Researchers have developed a novel device that has successfully detected lung cancer cells (Maheswaran, Sequist et al. 2008). Tumor cells in the blood of patients with lung cancer were captured, and run through a microfluidic device. The device consisted of 78,000 microposts that were coated with antibodies of the cancer cells. The cancer 
cells then adhered to the microposts during the capturing phase of the experiment. These microposts successfully isolated, quantified, and analyzed the captured tumor cells while the blood circulated the device.

\subsubsection{Application: Proteome Analysis}

Another important application of LOC is continuous isoelectric focusing (cIEF), and has recently become more prevalent in decoding of the human genome. Scientists are investigating the proteome, which is the complete qualitative and quantitative information about all proteins in a cell. Unlike the genome, the proteome is constantly changing, and scientists would ideally like to rapidly obtain information about its proteins quickly in order to analyze the changes it undergoes (Xu, Zhang et al. 2003). The genome focuses on the genes found in an organism, whereas the proteome studies the proteins in a specific cell. For the analysis, it is necessary to prepare, separate, identify and quantify thousands of protein samples. Currently existing methods of protein analysis include gel electrophoresis or spectrometry, which are slow and expensive, and do not yield the rapid results scientist would like to obtain. The current available method for a high-throughput analysis is performed using microwell plate-based assays and robotics (Xu, Zhang et al. 2003). A microwell plate is a rectangular plate made up of a matrix of wells that are used as miniature test tubes. Each well can hold from several nano liters to several milliliters of analytes. To integrate molecular analysis, sample preparation, reactions, separation and detection into a single microwell, plate, it would be necessary to incorporate hundreds of parallel channels in such a plate (Xu, Zhang et al. 2003).

The miniaturization of such a system could aid in eliminating the many processes needed for rapid protein separation using only a small sample, and to easily analyze the change the proteins in the proteome undergo. The 10-fold miniaturization of an electrophoretic experiment maintains the separation quality and same voltage applied in 
a conventional experiment, but results in a 100 time increase in timely efficiency, 10 times less reagents, and 1000 times smaller sample volumes (Liu, Xu et al. 2008).

\subsubsection{Application: Protein Separation}

Researchers are continuously making advances towards creating improved systems for cancer detection through identification and separation of biomarkers. The conventional approach for this separation involves running a complex sample through a two-dimensional gel electrophoresis (2-DE), and subsequently analyzing it using mass spectrometry (Hoffmann, Ji et al. 2001). The 2-DE technology is limited by the amount of protein that can be processed and the insolubility of certain classes of proteins, e.g. hydrophobic membrane proteins. Other shortcomings of the 2-DE is a combination of poor solubility in the current 2-DE buffers, the poor transfer efficiency from the gel-based IEF to the SDS-PAGE layer. During the process much of the sample can be lost and decreases the transcription of results (Hoffmann, Ji et al. 2001).

The similar technology of FFE (Free Flow Electrophoresis) on the other hand, has been proven to be successful in separating different analytes, including cells, cellular components, and proteins (Fonslow, Barocas et al. 2006). FFE uses the same principles as 2-DE, but in a more dynamic approach. FFE takes advantage of the particles' charge. A stream of analytes is injected in to a continuous stream of buffer between two electrodes. Once a potential is applied perpendicularly across the flow, the charged particles are deflected based on their mobility or IEF point. Since each analyte has different mobilities and IEF points, the initial single stream of analyte mixture is divided into individual streams composed of a pure analyte.

One advantage of FFE includes the collection of a large pure sample, since FFE is performed continuously. Another advantage of this separation method is that the 
separation is relatively gentile, and preserves enzymatic activity of the separands (Krivankova and Bocek 1998).

\subsection{Chip considerations based on prior research projects}

When designing a microfluidic chip, it is important to consider the effects of the surface properties of the chip materials, the electrodes and decreasing the effects of Joule Heating at the electrodes. All of these factors may compromise the experiment carried being carried out within the chamber. These may be addressed by varying the chamber heights by implementing the lubrication theory, and addressing the individual flow rates in the electrode and separation chambers.

\subsubsection{Pretreatment}

Methods of pretreatment include filtration, derivatization, and extraction (Liu, Garcia et al. 2003). More importantly, the three most common concentration methods are fieldamplified sample stacking, isotachophoresis, and solid-phase extraction (Walker and Beebe 2002).

Field-amplified sample stacking is accomplished by electrokinetically driving the sample from a region with a low buffer concentration into a region with a high buffer concentration. The difference in concentration causes the sample's velocity to decrease and for the sample to accumulate at the interface of the low and high buffer concentrations. Isotachophoresis is performed by having a sample positioned between a leading and trailing electrolyte. The leading electrolyte has a high electric mobility, and the trailing electrolyte has a mobility that is slower than that of the sample and the leading electrolyte. An electric current is then applied across the leading, sample, and trailing electrolyte, causing the sample to migrate depending on its electric mobility. Since each analyte in the sample has a different mobility, each analyte will be separated 
into individual bands between the electrolytes. In solid-phase extraction, the surface is treated with solids that attract the desired molecule in the sample solution. The sample solution is then allowed to flow across the treated surface of the device, and the targeted molecules are trapped on the surface, and can then be collected and analyzed (Walker and Beebe 2002).

\subsubsection{Joule Heating}

Original FFE devices had large separation chambers ( 25ml), but the low surface-tovolume ratio prevented the heat generated by the electrodes to dissipate efficiently. Due to the high temperature during the experiment, the maximum potential to the device was limited, which also decreased the efficiency of the experiment and the separation of the analytes (Fonslow and Bowser 2005). By miniaturizing the device, the surface-tovolume ratio decreased, and a higher high potential can be applied to the electrodes.

Joule heating occurs when an electric field is applied across a conductive medium, and the electrons interact with the surrounding ions. The charged particles accelerate in the medium and collide with ions, during which their kinetic or vibrational energy is transformed into heat energy. Therefore, at a higher solution concentration, a higher amount of Joule heating will take place. This can potentially cause reduction in separation efficiency, reduce the amount of analytes in the separation chamber, and

cause thermally sensitive analytes to decompose (Tang, Yan et al. 2006). In microfluidics, Joule heating leads to gas bubble formation at the electrodes. The gas bubbles are mainly hydrogen bubbles (Hong, Park et al. 2010) formed by electrolysis of water $\left(\mathrm{H}_{2} \mathrm{O}\right)$ at the cathode represented by the following chemical reaction: 


$$
\begin{array}{lrl}
1 H^{+}+2 e & \rightarrow H_{2} & \text { Equation 1 } \\
2 H^{+}+2 e \rightarrow H_{2} & \text { Equation 2 }
\end{array}
$$

The remaining negatively charged hydroxyl groups migrate to the anode to form oxygen:

$$
2 \mathrm{OH}^{-} \rightarrow \frac{1}{2} \mathrm{O}_{2}+\mathrm{H}_{2} \mathrm{O}+2 e
$$

The heating is represented by the power density, $Q$ in Volt/meter ${ }^{3}$ :

$$
Q=\frac{E \cdot I}{\pi \cdot r^{2}}
$$

$E$ represents the applied electric field, I represents the current, and $\mathrm{r}$ represents the radius of the capillary (Liu, Sosic et al. 1996). When applying this equation to a rectangular chip as later seen in this project (Section 2.5), the equation would be transformed to:

$$
Q=\frac{E \cdot I}{h \cdot b}
$$

$h$ and $b$ represent the height and length respectively of the separation chamber. To analyze the heat generated in a capillary, the following equation is applied:

$$
D T_{c}=\frac{Q \cdot r^{2}}{4 \cdot k_{b}}
$$

$D T_{c}$ represents the temperature difference between the capillary wall and the center of the capillary and $\mathrm{k}_{\mathrm{b}}$ is the conductivity of the buffer medium in W/cm K (Liu, Sosic et al. 1996). This leads to the conclusion that the larger the diameter of the capillary, the greater of the temperature difference between the capillary wall and the center of the capillary. Therefore, it is recommendable to decrease the cross-sectional area to decrease the amount of joule heating that can take place within the separation chamber. 


\subsubsection{Electrode Design}

A gold-sputtered electrode design was used successfully by previous a mylar IEF chip design (Macounova, Cabrera et al. 2000). A micromachined lift-off mask was fabricated to be placed on the mylar during the sputtering to create a design of the individual electrodes. The mylar was then treated with $\mathrm{O}_{2}$ plasma, and the $99.9 \%$ pure Gold was deposited on the material. The plasma treatment produced a strong bond between the gold and the mylar. Gold electrodes only allow for a voltage of $2.5 \mathrm{~V}$ or less to be applied to avoid the formation of bubbles. Using platinum or palladium electrodes could enable the application of a higher voltage (Macounova, Cabrera et al. 2000). Since this process is relatively expensive, this electrode design was not considered for this project.

Macounova, Cabrera et al. created a chip in which the electrodes were placed paralelly directly in the separation chamber (see Section 1.2.7.1) (Macounova, Cabrera et al. 2000). Despite the low potential that was possible to be used, the analytes adhered directly to the electrodes. Therefore, it is recommendable to implement a separate compartment for the electrodes to flush electrolysis products away, and to prevent analytes from contacting the electrodes.

\subsubsection{Hydrophilicity and Hydrophobicity of PDMS}

The main concern with the PDMS in microfluidic devices is that the material is hydrophobic, preventing effective fluid flow, and often trapping air in the chamber. Creating a hydrophilic surface is important, since it increases cell adhesion to the PDMS surfaces, and decreases bubble trapping. Some of the possibilities in gaining a hydrophilic surface include oxygen plasma treatment, the combination of oxygen plasma treatment and the constant contact with water, or coating the walls with polymers. 
Directly after the oxygen plasma treatment, i.e. after bonding the PDMS to glass, the surface is hydrophilic, but regains its hydrophobicity within hours (Eddington 2005). This hydrophobic recovery is due to the surface being thermodynamically unstable. The low molecular weight (LMW) non-crosslinked oligomers of the freshly cured PDMS diffuse to its surface, and render the surface hydrophobic (Vickers, Caulum et al. 2006). A different theory proposes that the hydroxyl groups located at the surface reorient themselves into the bulk by rotation about the sigma bonds. A portion of the surface charge is suspected to either due to stable $\mathrm{Si}(\mathrm{OH})$ or other moieties, since air-treated PDMS had twice the electrophoretic mobility of native PDMS (Ren, Bachman et al. 2001).

To analyze the changing surface properties of PDMS, it is possible to perform a Sessile drop test. During this test, a drop of water is placed on the surface of the material, and the contact angle between the curvature of the water drop and the surface is measured. This test was performed over several days on pristine PDMS and oxygen plasma treated PDMS. The contact angle of pristine PDMS was at $120^{\circ}$, while the newly treated material had a $0^{\circ}$ contact angle, that gradually increased over the period of 2 weeks to an angle of $105^{\circ}$ (Bodas and Khan Malek 2006). This research demonstrated that the hydrophobicity of PDMS increases over time after the initial casting.

In a different research project, EOF was analyzed by casting a PDMS device and immediately measuring the EOF, then casting a device, and measuring the EOF only several days later (Ren, Bachman et al. 2001). The relative standard deviation of the EOF velocities was $<10 \%$ when casted on the same day, whereas it was as high as $33 \%$ when testing the flow several days after casting. Since the repeated units of $\mathrm{OSi}\left(\mathrm{CH}_{3}\right)_{2} \mathrm{O}-$ are not expected to have a net surface charge, PDMS should not generate EOF. The origin of the surface charge on the material is relatively unclear, and 
researchers speculate that it stems from impurities in the material (cross-linking agents or silica fillers). Alternatively, it may also be explained that the surface properties of PDMS are different than the bulk, and that molecules located at the surface absorb surrounding molecules or undergo oxidative reactions (Momen and Farzaneh 2010). On non-treated PDMS, $-\mathrm{OH}$ groups were not detected on the surface, whereas PDMS undergoing oxygen plasma treatment showed that the $-\mathrm{Si}-\mathrm{CH}_{3}$ were transformed to $\mathrm{Si}^{-} \mathrm{O}^{-}$groups, rendering the PDMS surface hydrophilic. To preserve the hydrophilicity of the PDMS after the air plasma treatment, it is possible to maintain this surface property indefinitely with the constant contact with water or organic solvents (McDonald and Whitesides 2002).

Another method of maintaining a hydrophobic surface was explored by Eddington, et al., by applying thermal aging to the PDMS (Eddington 2005). It was hypothesized that low molecular weight (LMW) chains that migrate in PDMS to cover up its thermodynamically hydrophilic surface after the oxygen plasma treatment. Since the LMCs aid in creating a hydrophobic surface, it would be advisable to decrease the presence of these chains in the material. To decrease of the amount of LMW chains, the polymer is aged, since this causes the LMW chains to be volatized and thus removed from the bulk. In this study, Eddington et al. thermally aged samples PDMS at different lengths of time ranging from 0 to 14 days, and tested the hydrophobicity using the previously explained Sessile drop method. This study showed that that the longer the material was thermally aged, the longer the hydrophilicity of the PDMS was preserved.

The most common method of creating a hydrophilic surface on PDMS is by coating the walls with neutral and hydrophilic polymers. Researchers oftentimes use linear polyacrylamide for this process, but this substance does not withstand in alkaline 
environments. Additionally, this process has been proven to be time-consuming and highly labor intensive (Cui, Horiuchi et al. 2005).

\subsubsection{Restricting flow between Chambers}

In conventional free-flow electrophoretic devices, the electrode chamber is separated from the separation chamber using ion exchange, nylon, or cellulose membranes (Fonslow, Barocas et al. 2006). These barriers are permeable to ions, but the electrolysis products are flushed away by the electrode buffer. Since this is not possible to easily separate the separation chamber from the electrode chamber using these methods in microfluidic devices, other barriers such as salt bridges and varying chamber heights have been investigated.

\subsubsection{Channel Array}

A $4.07 \mathrm{~mm}$ wide, $12.22 \mathrm{~mm}$ long, and $10 \mu \mathrm{m}$ high separation chamber chip was investigated, which was supported by $30 \mu \mathrm{m} \times 30 \mu \mathrm{m}$ diamond shaped posts, spaced at a center-to-center distance of $40 \mu \mathrm{m}$ (Xu, Zhang et al. 2003). The electrode chamber was separated from the separation channel by $5 \mathrm{~mm}$ long and $4 \mu \mathrm{m}$ connection channels that were oriented transversely to the analyte flow direction. The numerous posts in the separation channel permit a small sample size, a quick analyte separation during the IEF experiment, and increased the surface-to-volume ratio; these are factors that aide in decreasing the effects of Joule heating. During the experiment, a successful separation of Fluorescein (2mM in $30 \% \mathrm{v} / \mathrm{v}$ ethanol) and Rhodamine-110 (1mM in $30 \% \mathrm{v} / \mathrm{v}$ ethanol) was carried out at $1750 \mathrm{~V}$ at $6 \mu \mathrm{L} / \mathrm{min}$. The separation took place within $75 \mathrm{~ms}$, at a very high resolution. When the voltage was increased to $4000 \mathrm{~V}$, electrolysis products began to form in the electrode chambers. 
Despite the quick separation time that was achieved with this chip, $90 \%$ of the voltage was lost due to the design of the connection channels (Xu, Zhang et al. 2003). The lack of a high voltage in the separation chamber would prevent a varied $\mathrm{pH}$ gradient from forming, and therefore prevent proteins with extreme IEF points to be used in an experiment (Raymond, Manz et al. 1996).

\subsubsection{Gel Barrier}

A different method of isolating the electrode chamber from the separation chamber is by incorporating a gel barrier within the chip. A PDMS chip was designed and fabricated using softlithography, and the $40 \times 40 \mu \mathrm{m}$ square posts spaced closely together separated the $20 \mathrm{~mm}$ long, $1 \mathrm{~mm}$ wide, and $50 \mu \mathrm{m}$ high separation channel from a layer of agar (Albrecht and Jensen 2006). Researchers used agar as a medium to separate the separation chamber from the electrode chamber, since agar is porous at a nano scale. The porous composition of the material would be permeable to ions, simultaneously act as a barrier to prevent fluid convection. The electrodes were embedded in the agar, thus allowing high voltages to be applied to the chip.

An experiment carried out with $200 \mathrm{~V}$ to focus IEF markers (at $\mathrm{pH} 3.5,5.1,7.2$, and 7.6). Joule heating caused the temperature inside the chip to rise to $42^{\circ} \mathrm{C}$ and was proven to be disruptive to focusing by causing Joule heating and creating electroosmotic flow (EOF) (Albrecht and Jensen 2006). Researchers applied a thermoelectric cooler to the top of the chip to decrease the temperature in the chip. If the temperature is too low within the sample chamber, condensation formed at the top of the glass cover slip, and

decreases the resolution of the fluorescence in the sample. Without the cooling mechanism, the voltage within the chamber was measured to be $520 \mathrm{~V} / \mathrm{cm}$, whereas the cooling lowered the potential to $140 \mathrm{~V} / \mathrm{cm}$. 
This design was not pursued, since it necessitated the use of a cooling element, which incorporated an additional variable in the experiment. Also, the main purpose of this project was to eliminate EOF, rather than performing IEF.

\subsubsection{Salt Bridge Barrier}

To isolate the electrode from the separation chamber, salt bridges were integrated in a microfluidic chip design (Kohlheyer, Besselink et al. 2006). A glass-glass microfluidic chip was designed (see Section 1.2.6.1), and injected with a solution consisting of a monomer (18\% acrylamide solution), crosslinker (N,N-methyulenebisacrylamide) and photoinitiator (2,2 dimethoxy-2-phylacetophanenone). A mask was placed over the chip to only expose the locations of the salt bridges to a mercury burner. The remaining solution was then flushed out, and the salt bridges were formed this way. These salt bridges formed a barrier for the pressure-driven flow, but still for ions to permeate the membrane, and thus form an electrical connection between the electrode and separation chamber. When testing this chip design for IEF, the salt bridges were functional for several hours, but soon broke apart. The failure was mainly due to the salinization bond of the salt bridges to the glass plate not being stable at extreme $\mathrm{pH}$ values. Although implementing salt bridges in the chip is a laborious process, the bubbles formed by electrolysis were contained in the electrode chamber.

\subsubsection{Using Channel Depth}

During cIEF, a constant stream of buffers in the electrode chamber and analytes in the separation chamber are applied. An innovative chip design used the channel depth to separate and control fluid flow in the separation and electrode channels (Fonslow, Barocas et al. 2006). Electrode channels being relatively much deeper than the 
separation channels aids in preventing electrolysis products from entering the separation channel.

The lubrication theory describes the flow rate, $q$, in a planar microfluidic channel,

$$
q=\frac{\Delta P \cdot H^{3} \cdot w}{12 n \cdot L}
$$

where $\Delta \mathrm{P}$ is the pressure difference, $\mathrm{w}$ is the channel width, $\mathrm{n}$ is the viscosity of the fluid, and $L$ is the channel length. Solving for the velocity, $v$, and modifying the lubrication equation, with $q=A \cdot v$ and $A=H \cdot w$, the linear velocity in the channel increases a 16-fold for a 4-fold increase in channel depth (Fonslow, Barocas et al. 2006):

$$
\begin{gathered}
v=\frac{\Delta P \cdot H^{2}}{12 n \cdot L} \\
16 v=\frac{\Delta P \cdot(4 H)^{2}}{12 n \cdot L}
\end{gathered}
$$

A deeper electrode chamber enables the electrolysis products to be flushed out rapidly while the fluid flow in the separation chamber is slower. The slower pace in the separation chamber allows for separations to take place without being influenced by the flow in the electrode chambers. This concept allows the design to steer away from creating a physical barrier separating the electrode and separation chamber, and thus a cheaper design.

\subsubsection{Material Selection}

The experiment carried out within the chip depends on the material of the chip itself. The main considerations of selecting the material include the visualization of the experiment through the chip, the bonding, workability of the material, endurance of the chip for repetitive testing, and the cost and availability of resources. 


\subsubsection{Glass Chips}

A free-flow zone electrophoresis glass-glass microfluidic chip was fabricated using a wet etching technique (Kohlheyer, Besselink et al. 2006). The chip consisted of two thermally bonded Borofloat-glass plates, and the bottom plate was left untouched. The features forming the chambers in the top-plate were created by sputtering a $140 \mathrm{~nm}$ layer of chromium-gold ( $\mathrm{Cr}-\mathrm{Au})$, which formed the mask. The channels and $15 \mu \mathrm{m}$ tall chambers were then etched by submerging the glass plate in a hydrofluoric acid bath. The etch glass plate was then thermally bonded to the untouched glass plate to form the microfluidic chip.

Since this process involves a complex etching technique, and requires a $\mathrm{Cr}-\mathrm{Au}$ mask, it is relatively expensive. This technology cannot be reused multiple times for testing and was deemed unsuitable the needs in this project.

\subsubsection{Polydimethylsiloxane Chips}

The first microfluidic devices used materials in which researchers were well-versed in, such as silicon and glass. These materials however are not efficient, when devices are needed for a rapid evaluation of prototypes, and are time-consuming and relatively expensive. To fabricate a microfluidic device, the main properties that are desired include ease of use, chemical stability, visibility, high resolution, and a low-cost material (McDonald and Whitesides 2002). Polydimethylsiloxane (PDMS) is transparent from 230-700 nm wavelength within the range of $190-700 \mathrm{~nm}$, which is excellent for optical monitoring and visible during lamination. The material polymerizes at a low temperature, seals readily with glass, PDMS, and other polymers. Since PDMS is also non-toxic and biologically inert, it does not damage living cells (Ren, Bachman et al. 2001). Additionally, it is easy to integrate mixers and switches into the PDMS chip. 
Due to the ease-of-use of PMDS, fabrication in this medium is preferred for prototypes. The soft-lithographic approach using a master, onto which PDMS is poured. The PDMS releases easily from the master, since the PDMS is relatively elastic, and has a low surface energy. The PDMS mixture typically has a 10:1 ratio of a base to curing agent. The curing agent contains silicon hydride groups, and combined with a base of vinyl groups. After curing, these two components form a clear cross-linked elastomeric solid. The 10nm resolution of PDMS allows for small features to be easily designed and fabricated (McDonald and Whitesides 2002).

\subsubsection{The $\mathrm{pH}$ Gradient}

Eliminating EOF is the first step to producing a microfluidic chip to IEF. The purpose of IEF is to focus proteins based on their surface charge, and the following methods to create a $\mathrm{pH}$ gradient have been investigated to create a basis for focusing of proteins. By using the same chip for the analysis of EOF and for future IEF testing, no new Si wafers will need to be commissioned for PDMS chip builds. This also facilitates the analysis of the test results, since the EOF testing will be performed on the same scale and chip design as any potential future testing.

It is possible to take advantage of the electrolysis products to create a pH gradient, whereas another method of creating a $\mathrm{pH}$ gradient can be performed by following traditional IEF closely by using ampholytes.

\subsubsection{1 pH Gradient through Electrolysis Products}

To create a natural $\mathrm{pH}$ gradient, a group used the electrolysis products generated by the anode and cathode. Relying on the $\mathrm{H}^{+}$generated at the anode, and $\mathrm{OH}^{-}$generated at the cathode, these molecules diffuse to the center of the channel (Cabrera, Finlayson et al. 2001). The requirements for these devices are a small distance between 
electrodes, diffusion and electrophoresis must be sufficiently quick to form a pH gradient. The device was made up $40 \mathrm{~mm}$ long gold electrodes that were placed $2.54 \mathrm{~mm}$ apart, with a $0.2 \mathrm{~mm}$ height forming the separation chamber. Since there was no physical barrier between the electrode and separation chamber, only $2.5 \mathrm{~V}$ needed to be applied to the device without losing any potential across a separation barrier. Due to the low potential, no bubbles due to Joule heating were formed in the separation chamber (Macounova, Cabrera et al. 2000).

This chamber design demonstrated a decreased convective flow. The benefits of a long and slim chamber design were that the small distance between the electrodes would reduce the amount of electrolysis products. Also, the surface-to-volume ratio would allow for heat generated by the electrodes to dissipate quickly and not inhibit the experiment in the separation chamber (Fonslow, Barocas et al. 2006). The lack of a high amount of heat generated by the electrodes, and the thin geometry of the chamber

decreases turbulent flow, and would improve the resolution of the experiment (Macounova, Cabrera et al. 2000).

Both Hemoglobin $(\mathrm{Hb})$ and Bovine Serum Albumin (BSA) conjugates were focused in the device, but during the experiment the amount of protein decreased, and a large portion adsorbed at the cathode. Due to the difficulty of analyzing the separation of the analytes because of the thin design of the chamber, and because the analytes were likely to adsorb to the electrodes, this design was not chosen.

\subsubsection{2 pH Gradient through Ampholytes}

The $\mathrm{pH}$ gradient is generated by carrier ampholytes, which are zwitterionic chemicals. Zwitterionic chemicals have the capacity to react either as an acid or a base. These ampholytes have a high buffering capacity near their pls, and are mixtures of hundreds of individual polymeric species with pls spanning a specific $\mathrm{pH}$ range. For 
example, water can be regarded as a zwitterionic chemical, and act as an acid or a base respectively:

$$
\begin{array}{ll}
\text { Acid: } \mathrm{NH}_{3(g)}+\mathrm{H}_{2} \mathrm{O}_{(l)} \leftrightarrow \mathrm{NH}_{4(a q)}^{+}+\mathrm{OH}_{(a q)}^{-} & \text {Equation } 10 \\
\text { Base: } \mathrm{HCl}_{3(g)}+\mathrm{H}_{2} \mathrm{O}_{(l)} \rightarrow \mathrm{H}_{2} \mathrm{O}_{(a q)}^{+}+\mathrm{Cl}_{(a q)}^{-} & \text {Equation } 11
\end{array}
$$

At the electrodes, the following reactions would take place at the anode (+) and cathode (-) respectively, where the anode would generate electrons, and the cathode would take in an electrode (Macounova, Cabrera et al. 2000):

$$
\begin{array}{cr}
\text { Anode: } \mathrm{H}_{2} \mathrm{O} \leftrightarrow 2 \mathrm{H}^{+}+\frac{1}{2} \mathrm{O}_{2}+2 e^{-} & \text {Equation } 12 \\
\text { Cathode: } 2 \mathrm{H}_{2} \mathrm{O}+2 e^{-} \leftrightarrow 2 \mathrm{OH}^{-}+\mathrm{H}_{2} & \text { Equation } 13
\end{array}
$$

When an electric field is applied, ampholytes that are the most positively charged (with the highest $\mathrm{pl}$ ) will migrate towards the cathode, while the most negatively charged ampholytes (with the lowest pl) will migrate towards the anode.

The remaining carrier ampholytes will align themselves between the extremes according to their pls, and buffer the environment to the corresponding $\mathrm{pH}$, forming a continuous $\mathrm{pH}$ gradient. The ampholyte migration will discontinue once they have reached their isoelectric point, and are no longer charged. The $\mathrm{pH}$ will therefore decrease towards the anodic section and increase towards the cathodic section.

The general composition of the ampholyte is:

$$
\mathrm{CH}_{2}=\mathrm{CH}-\mathrm{CO}-\mathrm{NH}-\mathrm{R}
$$

The R-group is usually composed of a carboxyl group $(-\mathrm{COOH})$ or a tertiary amine group (e.g. $-\mathrm{N}\left(\mathrm{CH}_{3}\right)_{2}$ ) (Davey and Lord 2003).

\subsubsection{Migration Distance}

In the most simple clEF chip designs, two different analytes are mixed, and then injected into the microfluidic chip. The stream of analytes is then isoelectrically focused 
in the separation chamber, and then collected. To facilitate the collection of analytes, the outlet placement of the separation chamber must be calculated. The migration distance, $D$, of the species can be determined with the following equation (Raymond, Manz et al. 1996):

$$
D=\frac{\mu \cdot I \cdot t}{A_{K}}=\left(\mu_{e p}+\mu_{e o}\right) E \cdot t
$$

The cross-sectional area of the across the separation bed, $A\left(\mathrm{~cm}^{2}\right)$, the carrier buffer conductivity the electroosmotic mobility of the analyte, $\mu_{e o}\left(\mathrm{~cm}^{2} / \mathrm{V} \cdot \mathrm{s}\right)$, the current, $I(A \mathrm{mp})$, the apparent mobility, $\mu\left(\mathrm{cm}^{2} / \mathrm{V} \cdot \mathrm{s}\right)$, the electrophoretic mobility, $\mu_{e p}\left(\mathrm{~cm}^{2} / \mathrm{V} \cdot \mathrm{s}\right)$, and the residence time, $t$ (sec).

\subsubsection{The Importance of Eliminating Electroosmosis in $\mu$ TAS}

By understanding $\mu$ TAS, and IEF, it becomes apparent that a stable environment must be maintained in the microfluidic chip. Due to the ion content in the analytes and buffers, the phenomenon known as EOF inevitably takes place at a microfluidic scale.

\subsubsection{Traditional 2-Dimensional Electrophoresis}

The principle of two-dimensional electrophoresis (2DE) has been widely employed in proteomics. The initial step of $2 \mathrm{DE}$ is to denature the proteins and to separate them based on their size. They can then be subsequently separated by their surface charge using IEF.

An electrophoresis apparatus works by setting up an agar or polyacrylamide gel, and applying an electric field across it. Since the proteins in the analyte have a surface charge, they will be repelled or attracted by the anode or cathode. The gel contains wells into which the analyte is dispensed, and a potential is applied across this gel. The larger molecules will move more slowly through the gel, whereas the smaller molecules 
will move faster. When the potential is then stopped, the molecules will stop migrating, and can then be collected.

The gel medium in which the electrophoresis is conducted can be placed in a tube, which is beneficial for one dimensional separations, or layered in flat sheets for experiments requiring two dimensional separation. The neutral, hydrophilic gels are formed by combining acrylamide and N,N-methylene0bis-acrylamide, and the polymerization is initiated by adding ammonium persulfate, which determines the pore size of the gel (Weber and Osborn 1969). The pore size of the gel is tailored to the protein size to be analyzed during the procedure. The analytes are combined with sodium dodecyl sulfate (SDS), which renders the proteins with a negative charge. The gel is poured into a mold that has wells positioned on one end, designated for the analytes. The electrodes are then positioned at opposite ends of the gel.

This technique is the initial step of separating the analyte mainly based on the molecular size. From this point, the proteins can be collected and used for further analysis, e.g. in IEF.

\subsubsection{Iso-Electric Focusing}

IEF is a type of electrophoresis and allows for the separation and concentration of compounds, in particular proteins, according to their intrinsic surface charge and molecular weight.

The initial step is to create a $\mathrm{pH}$ gradient across a medium by using carrier ampholytes (Probstein 2003). The ampholyte composed of a mixture of zwitterionic molecules; the negatively charged molecules will migrate towards the anode, while the positively charged molecules will migrate towards the cathode. This creates a $\mathrm{pH}$ gradient, since the cathode will have a larger quantity of positively charged ions, the $\mathrm{pH}$ level will be higher, and a lower $\mathrm{pH}$ will form at the anode. When the voltage is applied, 
the ampholyte will migrate until it reaches a point between the electrodes, at which it becomes neutral, which is known as its IEF point, pl. Therefore, the higher the number of ampholytes in a solution, the smoother the $\mathrm{pH}$ gradient becomes.

Microfluidic chips often use the IEF basics and apply these to a continuous flow, or semi-continuous flow through the microfluidic chamber. A semi-continuous flow was originally planned for this project and involved inserting all analytes, ampholyte and buffers in the chamber, stopping the flow, then subsequently running the IEF experiment. In continuous IEF (clEF), the focusing of analytes is carried out by supplying a steady stream of buffers, analytes and ampholytes in the chamber.

\subsubsection{The Protein}

Each protein is a charged molecule, composed of negative, positive, and neutral side chains, similarly to the ampholyte. The amino acids are held together by strong covalent bonds along the backbone, and weaker non-covalent bonds along its cross connections (Laberge 1998). The pls of proteins mainly lie between $\mathrm{pH} 3$ and $\mathrm{pH} 12$, with the majority lying between $\mathrm{pH} 4$ and pH 7 (Davey and Lord 2003). At the protein's pl, the molecule's net charge is zero, at which it will no longer migrate. Its pl is dependent on the net sum of the charges of the protein's side chains. The protein is positively charged below its pl, and negatively charged above its pl. Therefore, during electrophoresis, the protein will move towards the cathode when it is in a solution below its pl, or migrate to the anode when placed in a $\mathrm{pH}$ level above its $\mathrm{pl}$, until reaching its $\mathrm{pl}$, where it is rendered immobile.

\subsubsection{Electroosmosis}

The main concern with a semi-continuous IEF experiment is stopping all flow in the chamber to allow the proteins to focus based solely on their surface charge. When 
conducting experiments in microfluidic chips, especially in PDMS chips, the surface layer at the fluid/chip interface will cause a phenomenon known as electroosmosis. This leads to the bulk movement of the fluid contained in the chamber, which influences the migration of the analytes during the IEF.

Since dielectric materials such as PDMS and glass have an inherent electric charge at their interface when in contact with an aqueous medium (Probstein 2003), an electric double layer (EDL) form at the surface (Baldessari and Santiago 2006). The EDL is formed by the ions on the surface of the material attracting its counterions (ions of opposite charge of the material) from the aqueous solution, bringing a higher concentration of these counterions to the material-solution interface, while the solution's coions (ions of the same charge as the material) are repelled (Probstein 2003). The thin layer of counterions immediately next to the charged solid surface is called the compact layer (Sze, Erickson et al. 2003), and the potential at the EDL is known as the zeta potential. The thickness of the EDL depends on the ion concentration in the electrolytes. Therefore, the surface of the PDMS is shielded by a layer of counterions from the solution. A portion of the counterions will condense on the surface of the material, neutralizing the surface charge, while the remaining portion will remain soluble and diffuse in the solution (Baldessari and Santiago 2006).

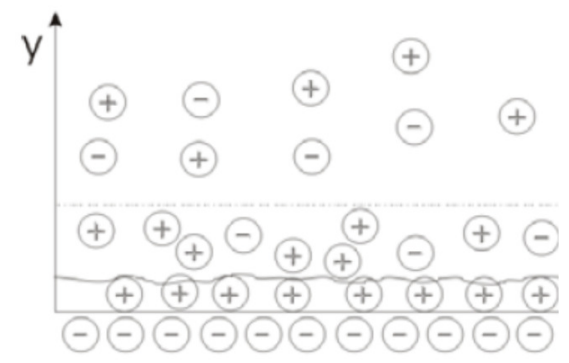

Figure 1: Ion constitution at fluid/folid interface (Breuer 2005) 
As seen in Figure 1, the larger concentration of counterions remains near the surface of the material, while the larger concentration of coions remains towards the center of the solution. The flow of electricity is from the positive electrode to the negative electrode, thus creating an electric field oriented towards the cathode. Positively charged ions move faster than negatively charged ions, resulting in a net flow to move towards the cathode.

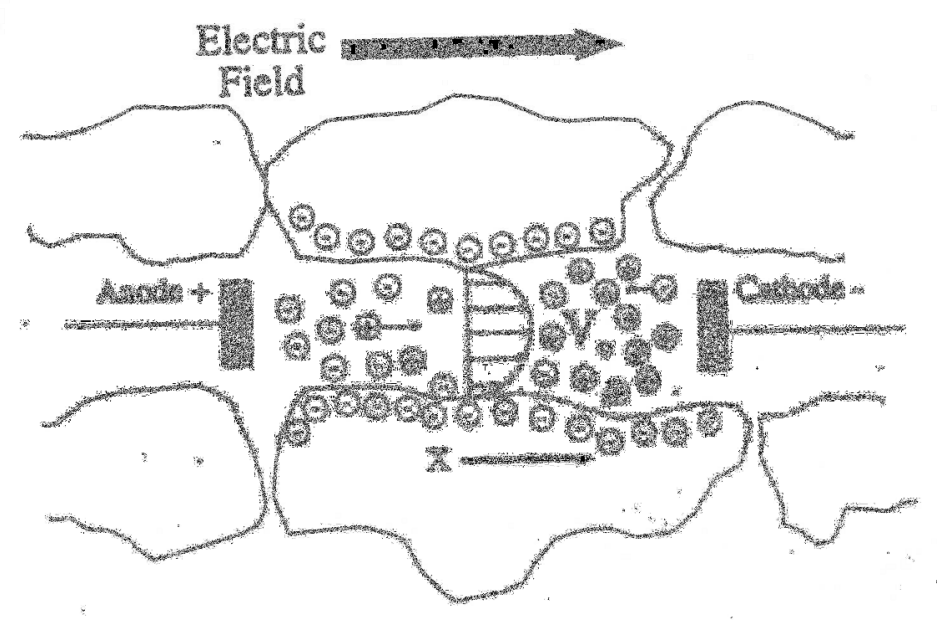

Figure 2: Velocity Profile of EOF (Probstein, 2003)

A main problem with microfluidics in PDMS chips is the electroosmotic flow (EOF) because of the material's hydrophobicity. During electroosmosis, the aqueous solution moves towards cathode (Probstein 2003), which is problematic, since this may also cause the analytes to migrate towards the cathode.

The zeta potentials of PDMS and glass were measured to lie in the range of -110 to $68 \mathrm{mV}$ and -88 to $-66 \mathrm{mV}$ respectively (Sze, Erickson et al. 2003) depending on the electrolyte solution. It was also concluded in the same study that zeta potential of glass and PDMS are independent of the chamber height and magnitude of the electric field.

In an ideal case, the surface charge of the material would be eliminated and set to zero, which may be approximated by plasma-treating the surface. But PDMS oxidates, and hydrophilic surface cannot be maintained when exposed to air (Ren, Bachman et al. 
2001). The following equation describing electroosmosis developed by Hjerten (Cui, Horiuchi et al. 2005) for capillary electrophoresis can also be applied to rectangular channels:

$$
\mu_{e o}=\frac{\epsilon}{4 \pi} \int_{0}^{\xi} \frac{1}{\eta} d \psi
$$

Where $\epsilon$ is the dielectric constant, $\xi$ is the zeta potential, $\psi$ denominates the electric potential at a distance $\mathrm{x}$ from the wall, and $\eta$ is the viscosity in the electrical double layer. By analyzing this equation, it would be possible to reduce the electroosmotic flow by increasing the viscosity of the fluid, since

$$
\lim _{\eta \rightarrow \infty} \mu_{e o}=0
$$

Equation 17

The velocity of EOF can also be approximated by the following equation:

$$
v_{E O F}=\frac{\varepsilon \zeta}{\mu} \cdot E
$$

$\varepsilon$ is the dielectric constant of the fluid, $\zeta$ is the zeta potential, and $\mu$ is the viscosity (Fu, Lin et al. 2003), E is the applied electric field intensity.

When bonding PDMS to the glass substrate, oxygen plasma is used on both surfaces as later discussed in Section 2.5.7. However, research reveals that when applying this surface treatment to the surfaces, the chip is easier to fill with aqueous solutions than when the surfaces are left untreated (Ren, Bachman et al. 2001).

The common method of eliminating the protein adsorption to the PDMS, is by treating the chip with methylcellulose. Per Equation 18, it would be possible to decrease EOF by using an analyte with a high viscosity. A possibility of increasing the viscosity of the material's surface would be to coat the surface with methylcellulose, as previously done by (Cui, Horiuchi et al. 2005). This coating procedure was used during a dynamic coating in a clEF separation 
In this project, the surfaces of the PDMS chip will be coated with Methylcellulose to attempt to decrease EOF. 


\section{MATERIALS AND METHODS}

\subsection{Chip Design}

Since this chip is designed with the intent to be used on a continuous IEF process, preliminary chip designs were investigated using COMSOL to analyze the flow behavior and electric conductivity.

\subsection{Preliminary Design: Electrode Channel Array}

The primary obstacle of the microfluidic chip's preliminary design is to eliminate is the generation of electrolysis products in the electrode chambers. The electrolysis products are bubbles generated by the electrodes in the electrode chambers that decrease the potential of the electric field and the flow of the buffer in the separation chamber. This prevents the correct functioning of the chip and the separation of proteins. In conventional FFE devices, the separation chamber is physically separated from the electrode chamber by nylon mesh, ion exchange, or cellulose membranes (Fonslow, Barocas et al. 2006).

In a silicon chip design with a glass substrate, channel arrays oriented perpendicularly to the direction of flow was used to isolate the electrode chamber from the separation chamber (Raymond, Manz et al. 1996). The dense channel array created a high hydrodynamic resistance, preventing the electrolysis products from entering the separation chamber. This chip design was beneficial to secluding the electrolysis products to the electrode beds, but decreased the separation efficiency in the experimental chamber (Kohlheyer, Besselink et al. 2006). Although no EOF was observed due to the transverse channels, convection from the electrode chamber into the separation chamber decreased prevented the separation of analytes. After further 
investigation, incorporating a channel array in the chip design was too costly for the scope of this project.

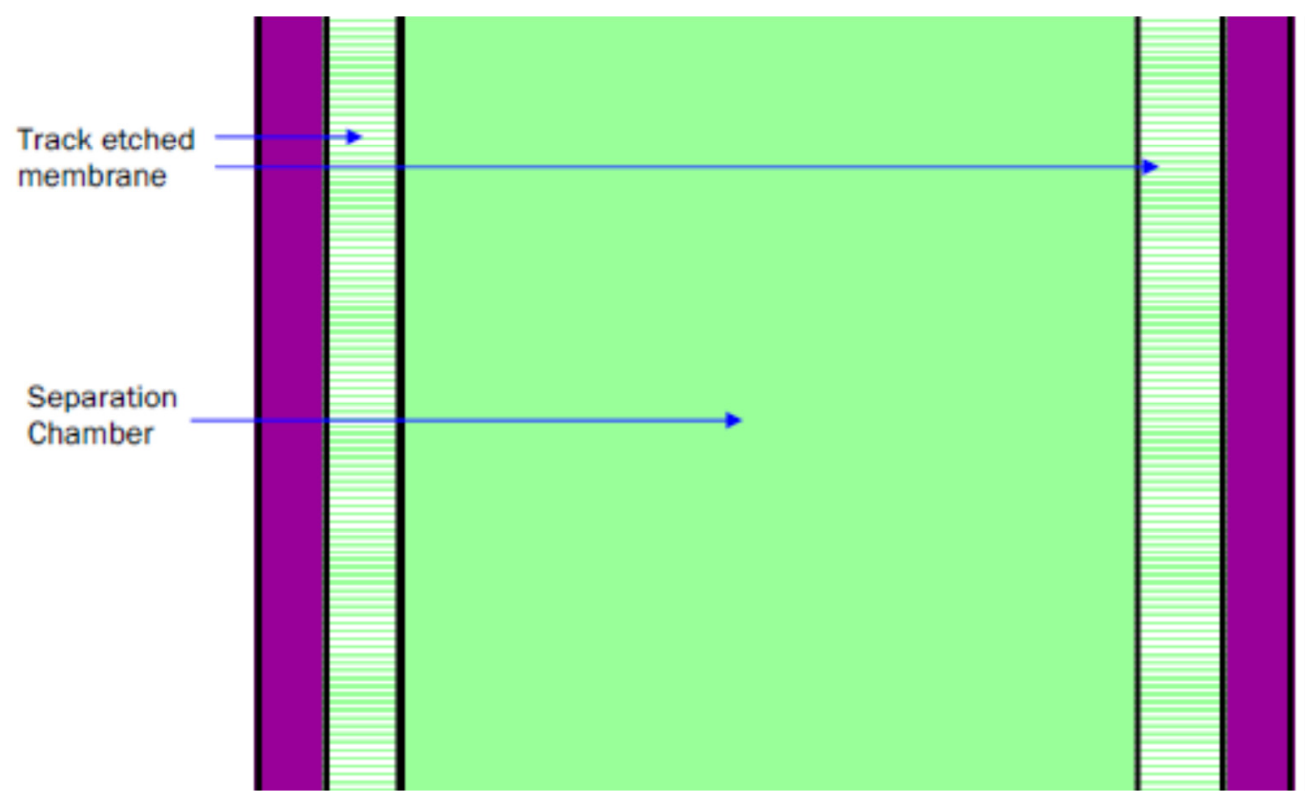

Figure 3: Channel Array Design- Schematic

In a different experiment, a similar design was used and the channel arrays were implemented by designing a PDMS chamber on a glass substrate, a higher electric field strength, flow velocities, separation efficiency and throughput (Zhang and Manz 2003). Due to the dense channel array, only $5 \%$ of the potential applied at the electrodes could be used for separating the analytes.

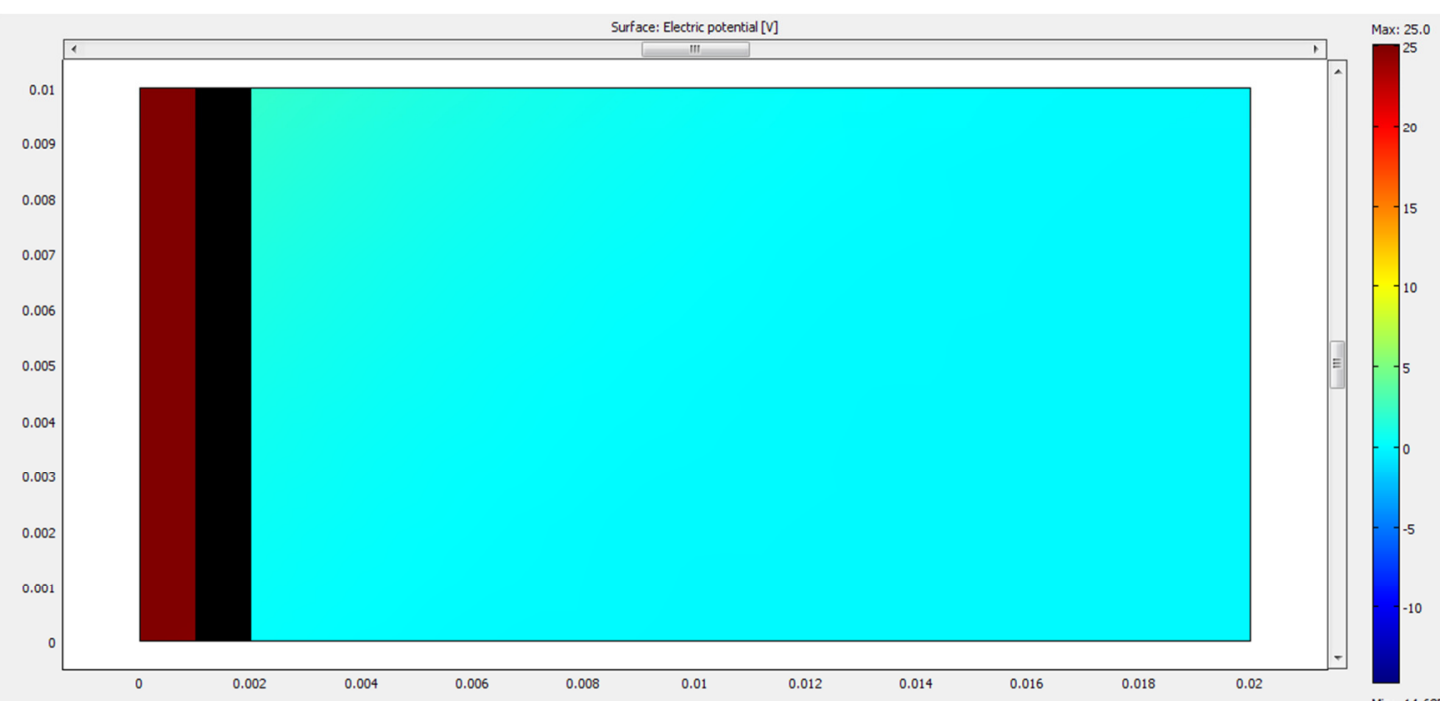




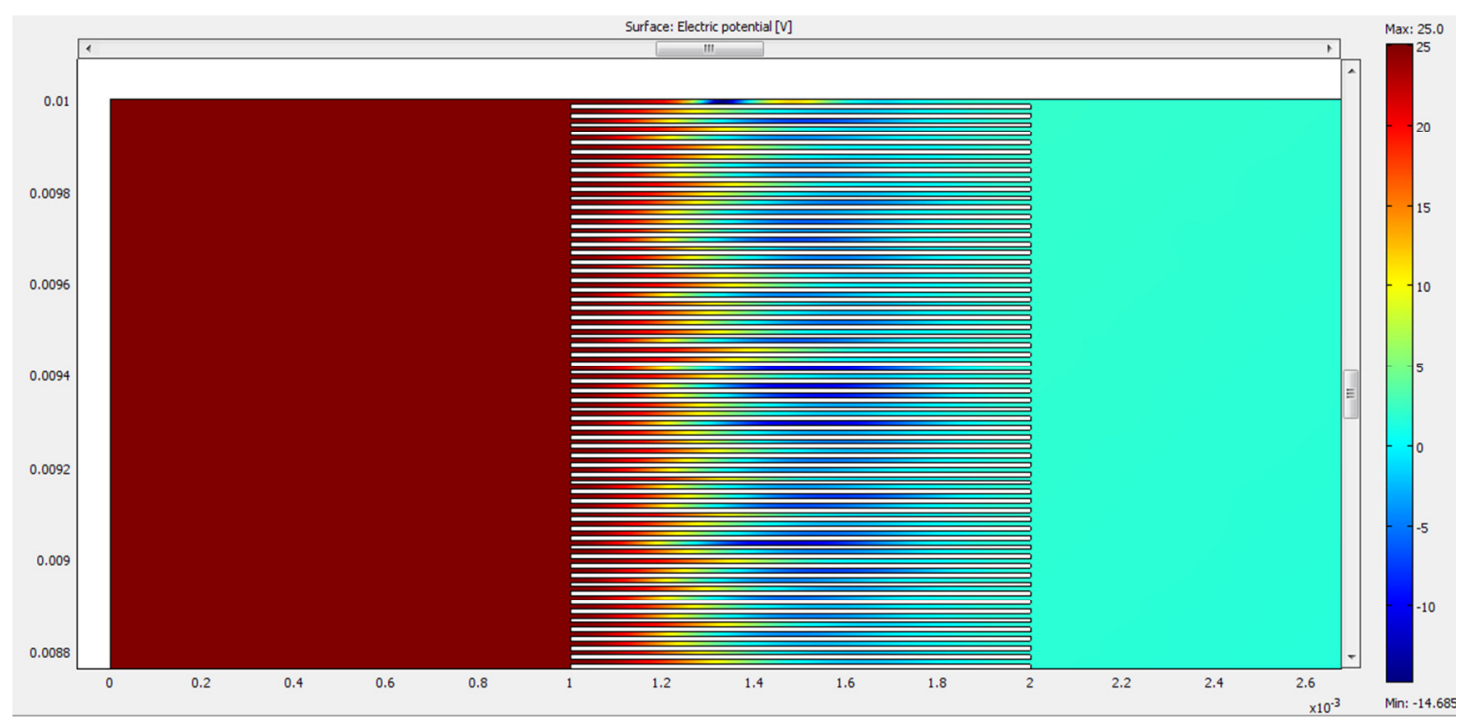

Figure 4: Channel Array Design- COMSOL modeling of overall view (above) and detailed view (below) of the electric potential across the chip

This preliminary design incorporated multiple $\mathrm{pH}$ inlets that were oriented parallel to the fluid flow. Each inlet was designed for a single $\mathrm{pH}$ buffer in order to create a $\mathrm{pH}$ gradient across the width of the chamber. Several weirs were incorporated in the design to collect the analytes after they are separated in the chamber (Raymond, Manz et al. 1996).

This design was modeled in COMSOL, as seen in Figure 4, and the drop across the channel array is as follows: 


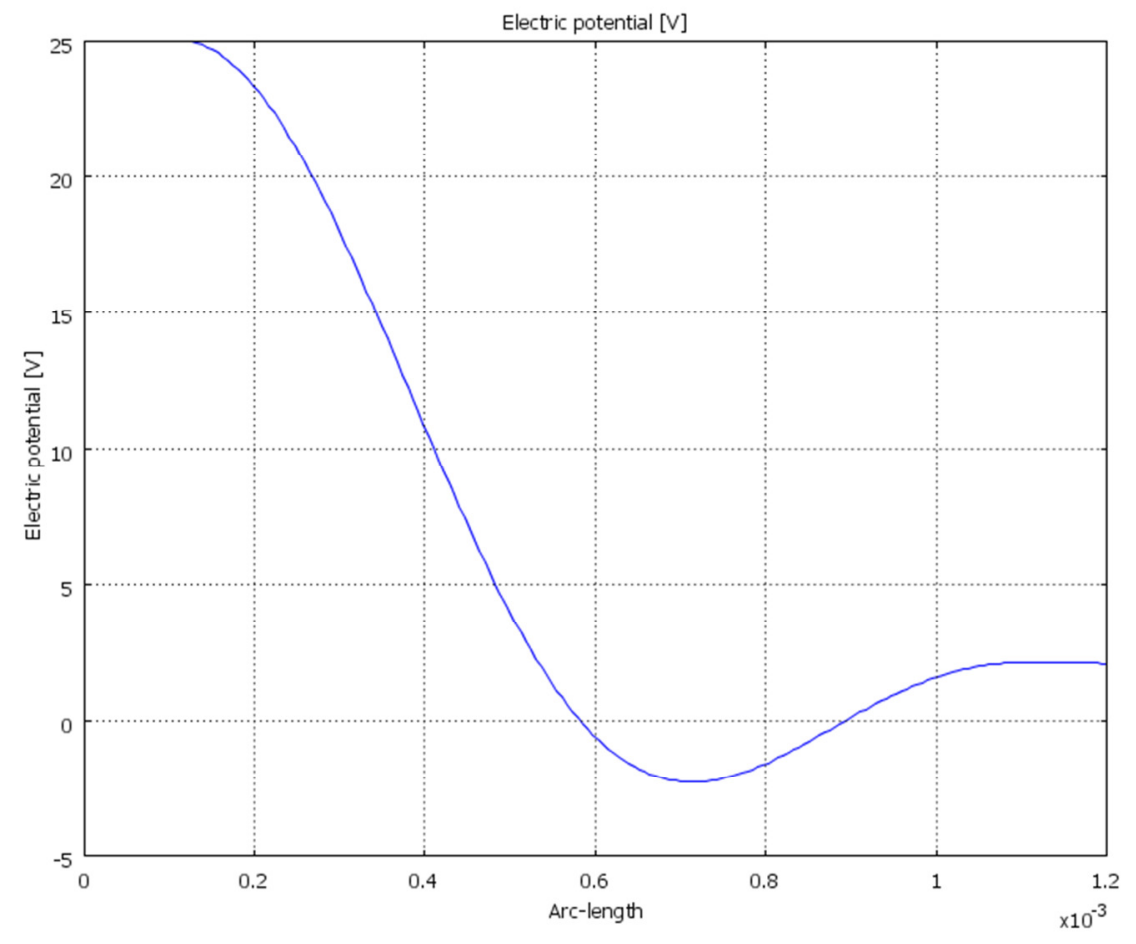

Figure 5: Channel Array Design- Voltage drop

As seen in the above Figure 5, the voltage in the COMSOL model dropped by approximately a 10-fold in this chip geometry.

\subsection{Preliminary Design: Separate Buffer Inlets}

The second preliminary design consisted of introducing ten separate entry channels in addition to the transverse channel array isolating the electrode from the separation chamber.

Each channel (Figure 6) would supply the separation chamber with a buffer at a different $\mathrm{pH}$. The goal of introducing the ten separate channels was to create a gradient that would remain constant throughout the length of the separation chamber. At the end of the separation chamber, there are different weirs designated for protein collection depending on their individual IEF points. 


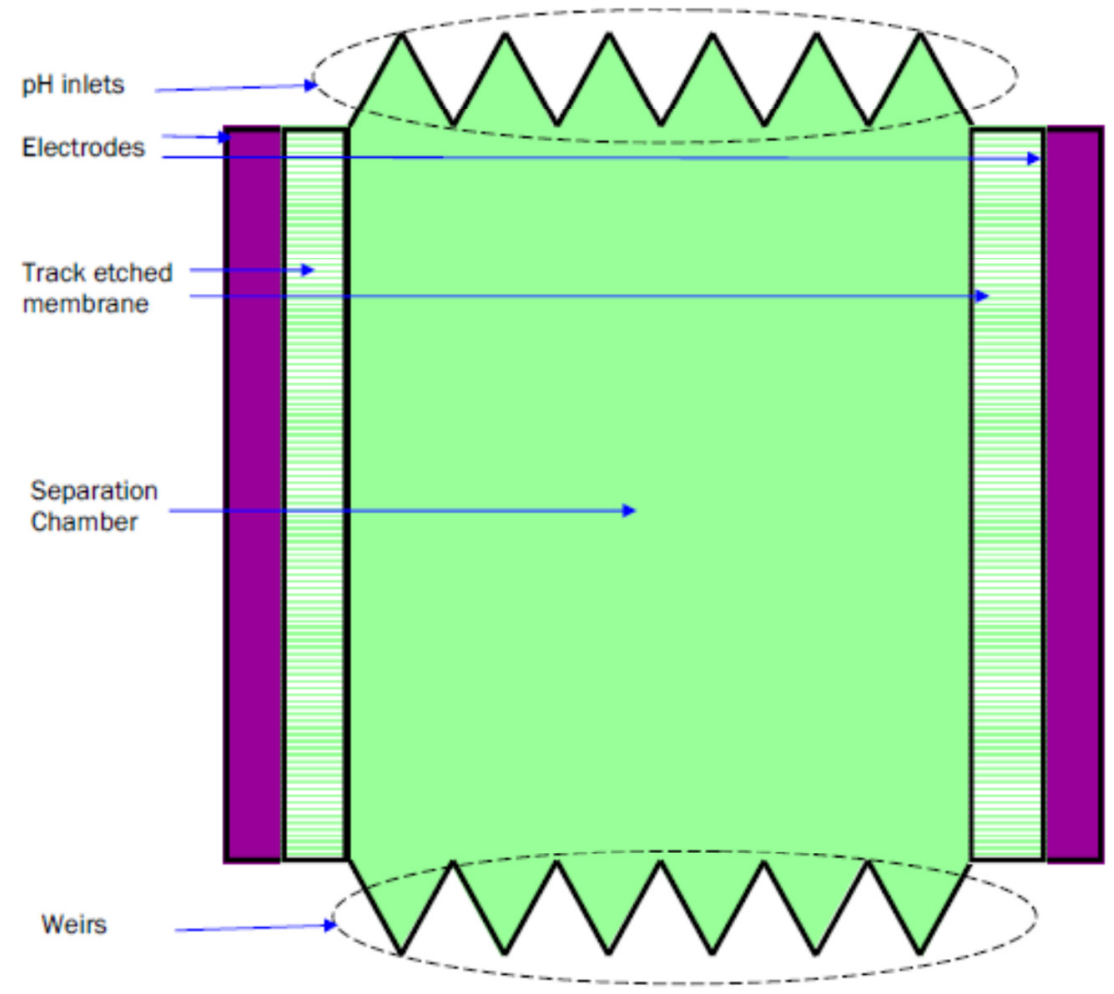

Figure 6: Design 2- Track etched membrane, separate pH buffer inlets, and multiple sample collection weirs.

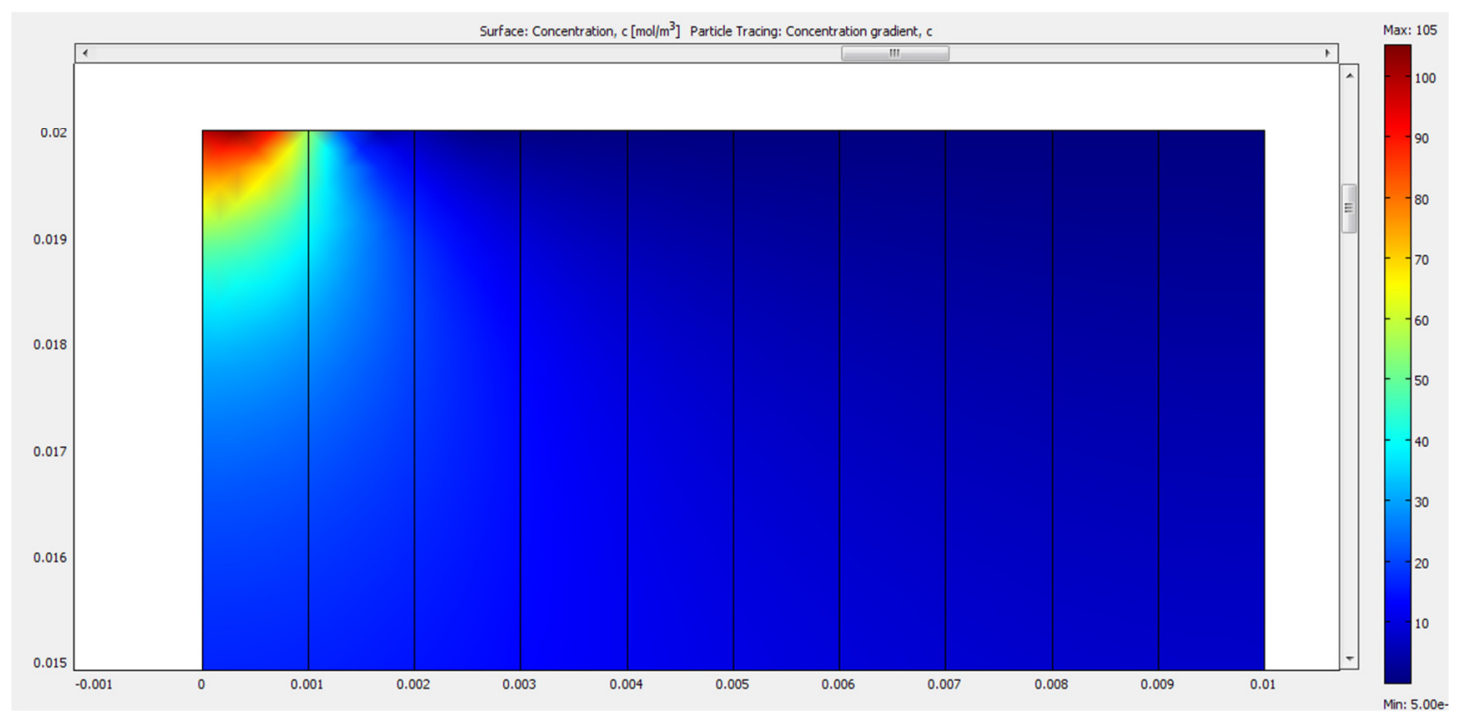

Figure 7: Separate Buffer Inlet Design- COMSOL modeling of $\mathrm{pH}$ level 
The COMSOL model shows that the concentration of $\mathrm{H}^{+}$ions representing the $\mathrm{pH}$ gradient quickly dissipated along the length of the chamber as seen in Figure 8. The concentration profile was analyzed at $0.001 \mathrm{~m}$ from the left edge of the chamber.

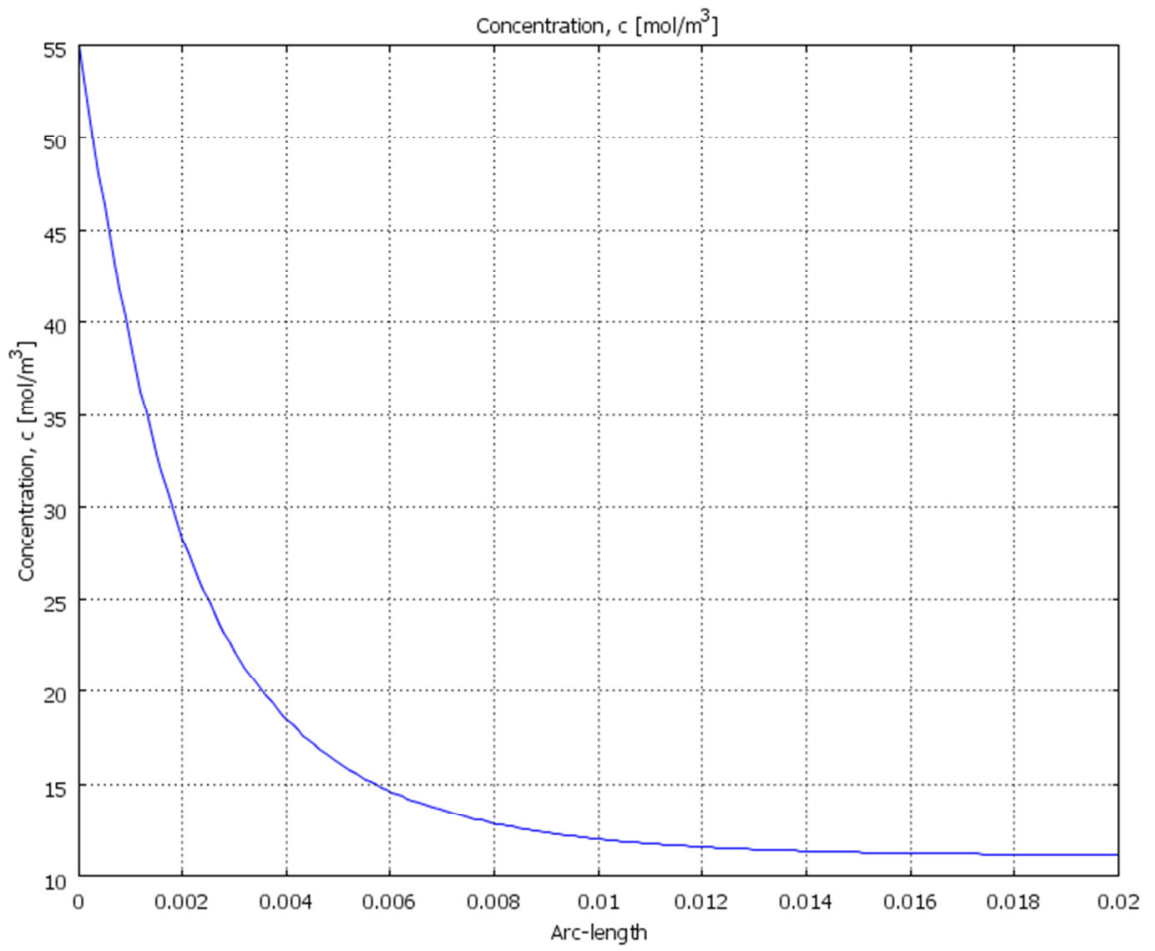

Figure 8: Separate Buffer Inlet Design- $\mathrm{H}^{+}$Concentration along the length of the chip Also, the concentration was analyzed across the width of the chamber: 


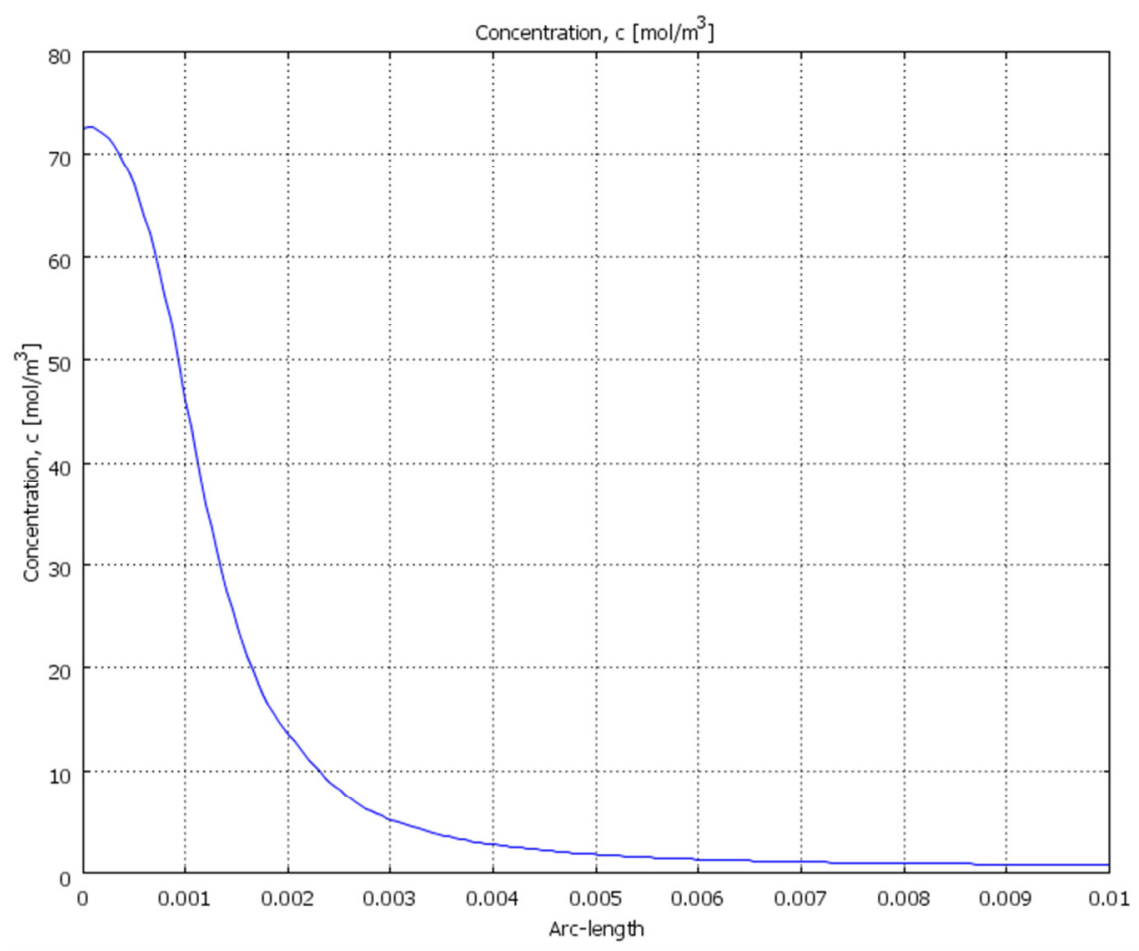

Figure 9: Separate Buffer Inlet Design- $\mathrm{H}^{+}$Concentration across the chip at $0.0005 \mathrm{~m}$ from inlet

The dissipation of the buffers led to the creation of a very narrow $\mathrm{pH}$ level in the separation chamber, as seen in Figure 10. 


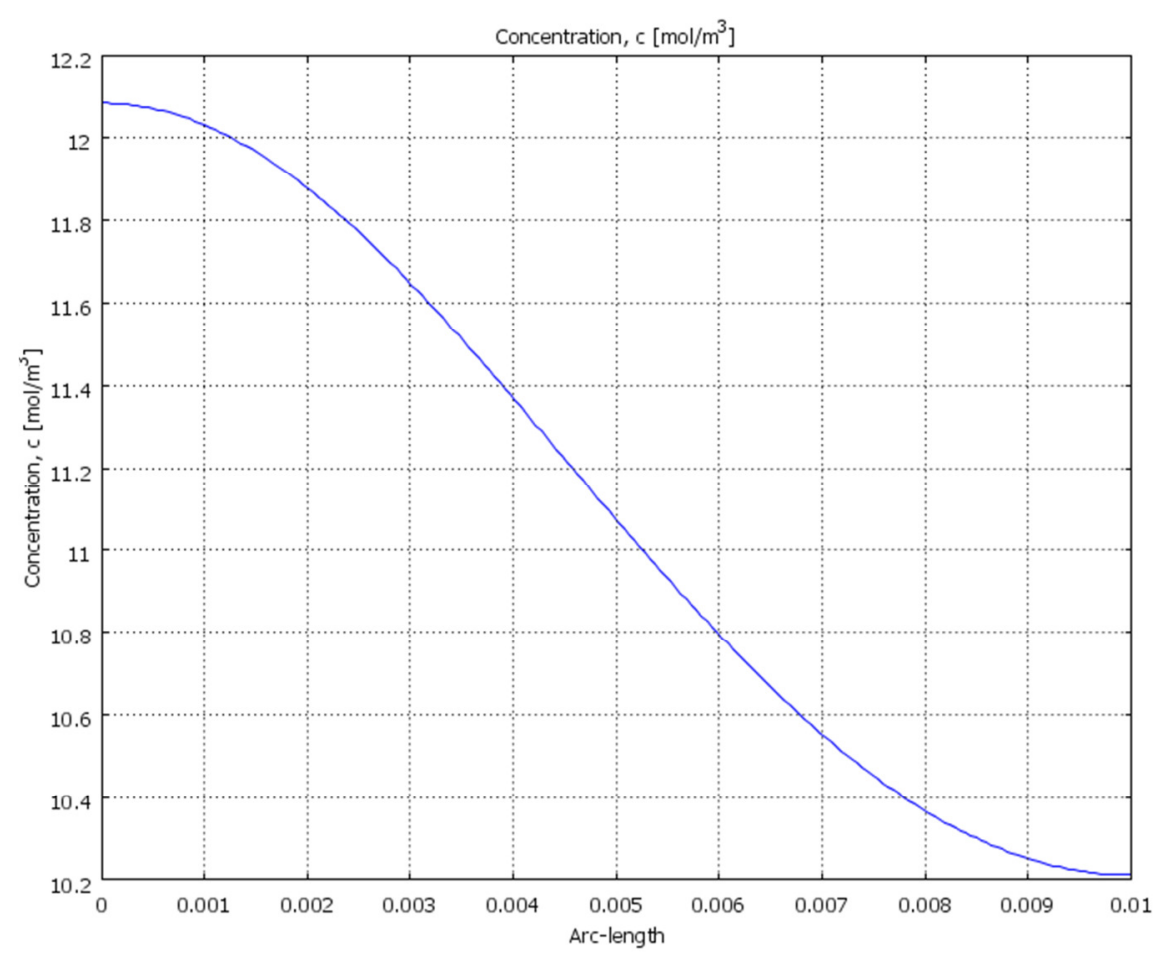

Figure 10: Separate Buffer Inlet Design- $\mathrm{H}^{+}$Concentration gradient across the width of the channel at $0.01 \mathrm{~cm}$ after the inlets

At $0.01 \mathrm{~m}$ downstream of the inlets, the concentration of $\mathrm{H}^{+}$ions varied from $12.1 \mathrm{~mol} / \mathrm{m}^{3}\left(12.1 \times 10^{-3} \mathrm{M}\right)$ to $10.2 \mathrm{~mol} / \mathrm{m}^{3}\left(10.2 \times 10^{-3} \mathrm{M}\right)$, the equivalent of a range of $\mathrm{pH}$ 2.9 to $\mathrm{pH} 3.0$ per Equation 19.

$$
p H=\log _{10}\left[H^{+}\right]
$$

Equation 19

\subsection{Preliminary Design: Channel Array Design}

The next preliminary design was modeled after a previously created design with inter-branching capillaries that enabled the control of the intensity and shape of a gradient (Dertinger, Chiu et al. 2001). The chip was composed of a pyramidal design of serpentine capillaries that met at nodes, where they were allowed to mix by diffusion with the neighboring streams. Using this design, individual buffers with specific $\mathrm{pH}$ levels could be introduced, and subsequently mixed at branching points. The location of 
the $\mathrm{pH}$ concentrations in the separation stream could be controlled by varying the chemical composition in the inlets.

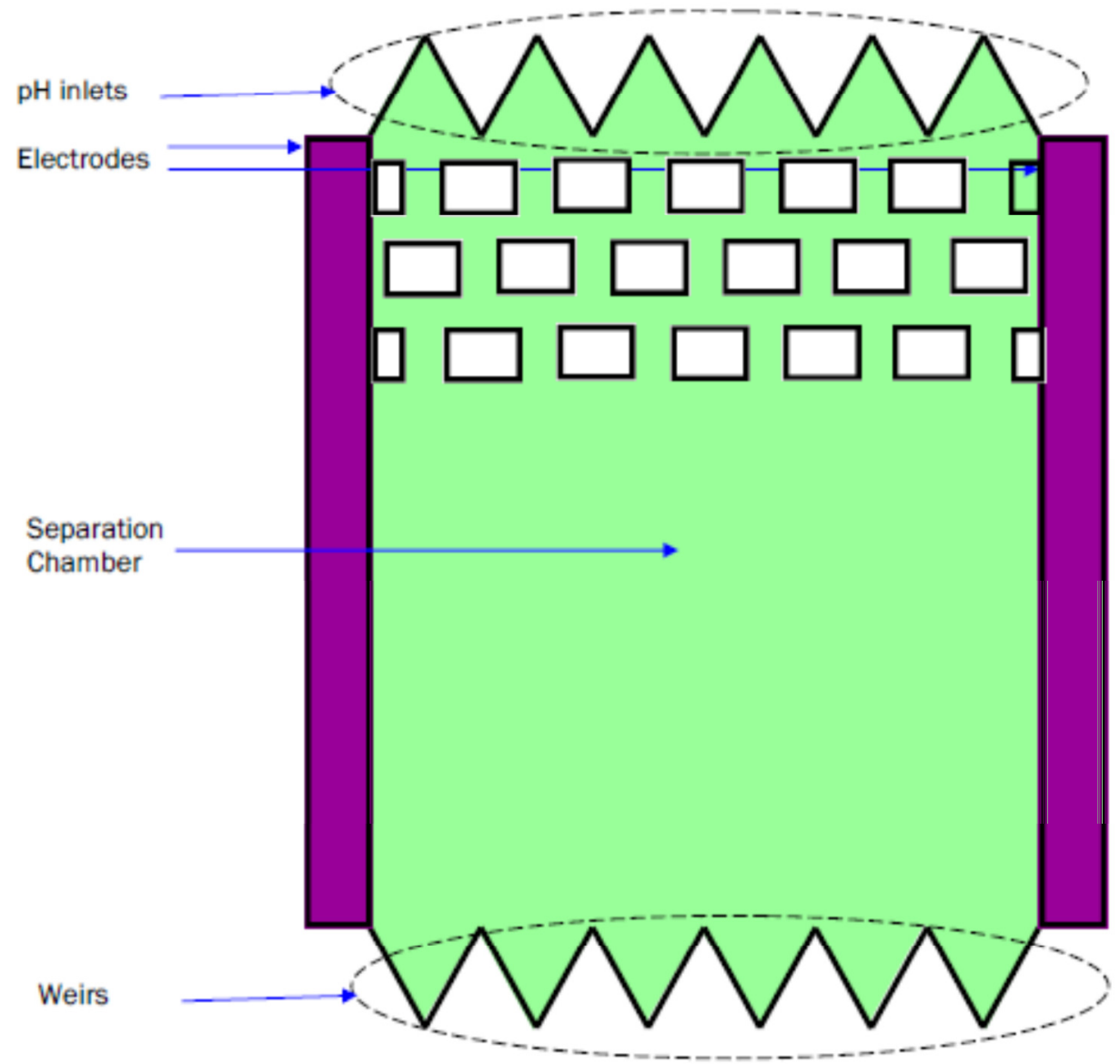

Figure 11: Channel Array Design- Schematic

This design was modified and simplified and the complex capillaries were replaced with simple channels that were separated by rectangular staggered blocks. The channels meet at the staggered nodes as seen in the drawing below. Ten different buffers with different $\mathrm{pH}$ levels are introduced separate inlets, and mixed within the staggered channels in the separation chamber. 


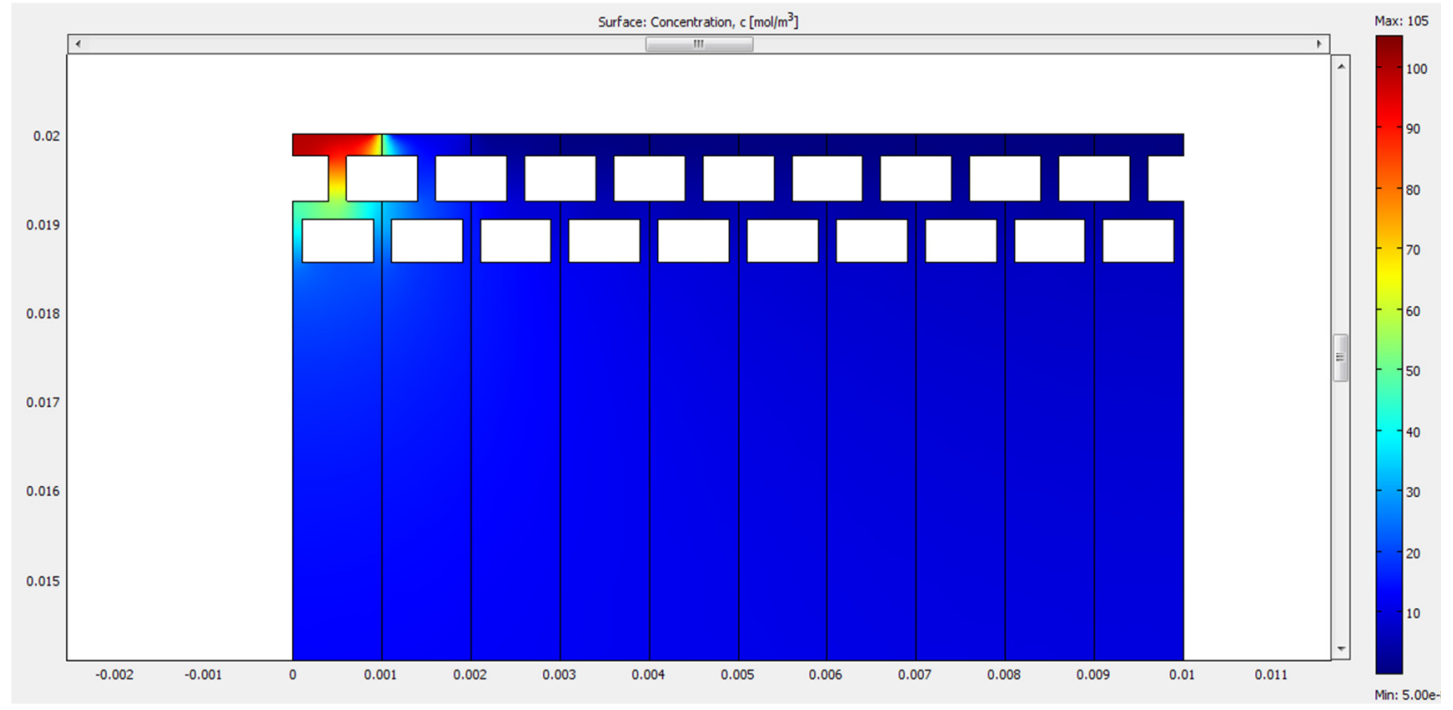

Figure 12: Channel Array Design- COMSOL modeling of pH concentrations

After modeling this design in COMSOL, this simplified version of the previously designed chip failed in creating a successful $\mathrm{pH}$ gradient. The number of $\mathrm{pH}$ buffer inlets was modified, as were the flow rates, and channel sizes.

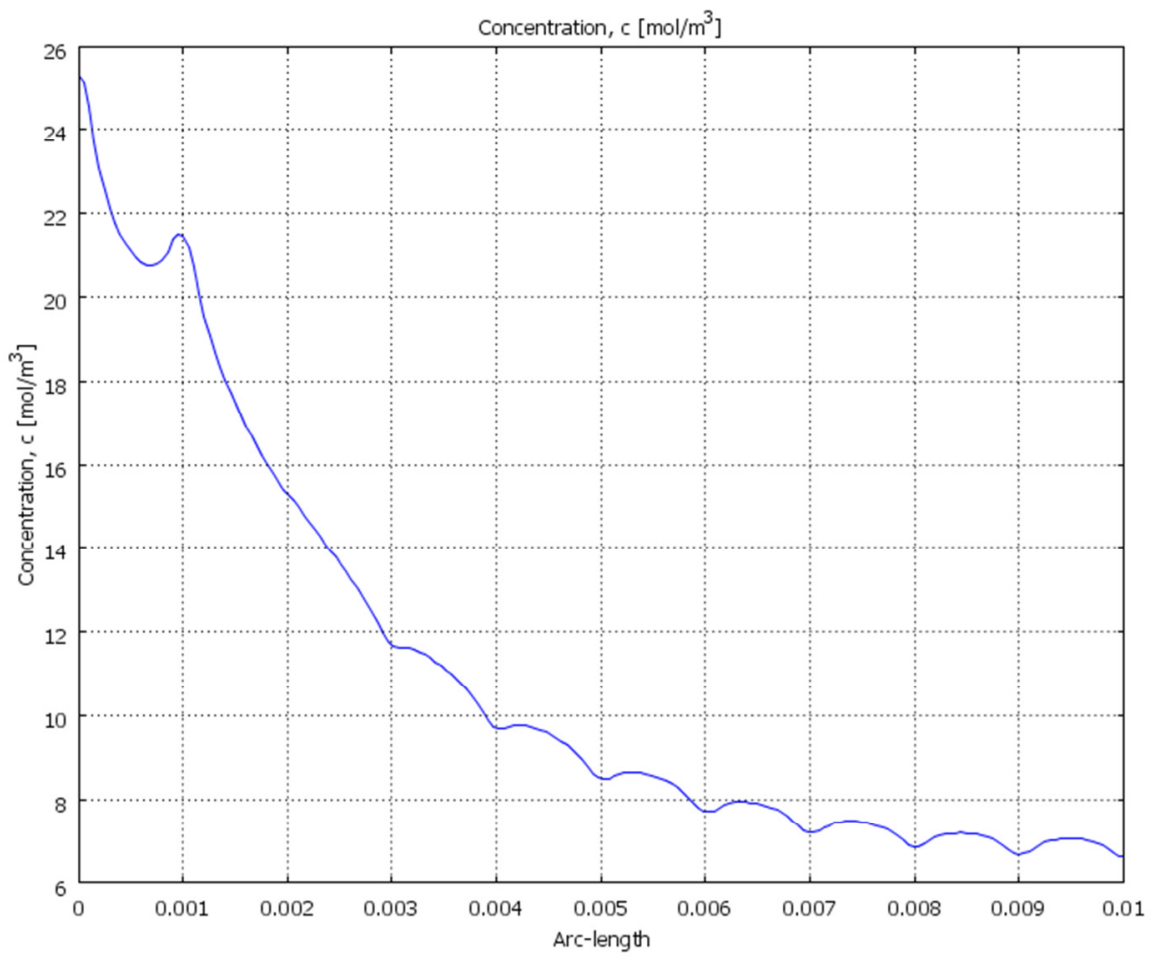

Figure 13: Channel Array Design- $\mathrm{H}^{+}$Concentration immediately after the staggered blocks 
At $0.0015 \mathrm{~m}$ downstream of the inlets, directly after the staggered blocks, the concentration of $\mathrm{H}^{+}$ions varied from $25.1 \mathrm{~mol} / \mathrm{m}^{3}\left(25.1 \times 10^{-3} \mathrm{M}\right)$ to $6.8 \mathrm{~mol} / \mathrm{m}^{3}\left(6.8 \times 10^{-3} \mathrm{M}\right)$, the equivalent of a range of $\mathrm{pH} 2.6$ to $\mathrm{pH} 3.2$ per Equation 19.

As with the preliminary Design II, the $\mathrm{pH}$ gradients formed by the different buffers quickly dissipated, creating a narrow pH gradient in the separation chamber.

\subsection{Final Chip Design}

The original chip design was geared toward creating a cIEF chip, and was made up of electrode and separation chamber inlets and outlets. The designs were specifically tailored for the separation of Bovine Serum Albumin and Bovine Hemoglobin, and using channel depth to isolate the separation chamber contents from the electrode chamber analytes.

Since the silicone wafer was large enough to incorporate two chip designs, two similar designs were construed. Originally, the chip design geared towards IEF, and included one inlet for the proteins, and a separate inlet for the ampholytes. The first design (Chip1) has a wider chamber, whereas the second design (Chip 2) has a more separation chamber. The wider design enables a wider gradient to form, which could facilitate the visualization and documentation of the separation, and potentially allow for several different proteins to be focused. The second design would allow for a simpler separation or focusing to be performed, using only one or two proteins.

\subsubsection{Analytes}

The future of research in this field lies in the separation and identification of specific proteins in a complex fluid at a microfluidic level. To demonstrate this separation via IEF in a complex fluid, a simplified version was created to test this hypothesis. The proteins Bovine Serum Albumin (BSA) and Bovine Hemoglobin $(\mathrm{BHb})$ are recommended for 
experimental purposes because of their differing IEF points $(\mathrm{pl})$. The protein selection of the current project was based on previous research conducted by Macanouva et al., with the well-known pls of the proteins noted at 4.65 for BSA, and 7.1 for BHb (Macounova, Cabrera et al. 2000). These were also recommended because they exhibit excellent characteristics to be focused into narrow streams. The chip design previously described in Section 2.5.1 is based on the pl of these two proteins.

\subsubsection{Inlet and Outlet Placement}

The inlet was placed at the top left of the chip near the cathode, since the electric field would cause the analytes to migrate towards the anode.

The placement of the outlets was based on the migration distance, if $\mathrm{Hb}$ and BSA were to be focused. To easily collect the $\mathrm{Hb}$ and BSA samples, the outlets would be placed at the location at which the proteins would reach their IEF point (pI). The ampholytes were assumed to take on an even distribution across the separation chamber, and form into ten ( $n_{\text {ampholyte }}$ ) evenly spaced bands ranging from $\mathrm{pH}$ 3-10. Based on this assumption and knowing that the pls proteins Bovine Serum Albumin (BSA) and Bovine Hemoglobin (BHb) have $\mathrm{pl}_{\mathrm{BSA}}=4.65$ and $\mathrm{pl}_{\mathrm{BHb}}=7.1 \mathrm{BHb}$ (Macounova, Cabrera et al. 2000), the outlet placement $\left(D_{\text {outlet }}\right)$ from the cathode were placed at the $\mathrm{pl}$ of the proteins per the following calculation and by using the variables listed in

Table 1:

$$
D_{\text {outlet }}=\frac{p I / n_{\text {ampholyte }}}{w}
$$

Table 1: Outlet placement variables.

\begin{tabular}{ll}
$\mathbf{p l}_{\mathrm{BSA}}$ & 4.65 \\
$\mathbf{p l}_{\mathrm{BHb}}$ & 7.1 \\
$\mathbf{n}_{\text {ampholyte }}$ & 10 \\
$\mathbf{w}_{\text {Chip2 }}$ & $1 \mathrm{~cm}$ \\
$\mathbf{w}_{\text {Chip2 }}$ & $2 \mathrm{~cm}$ \\
\hline
\end{tabular}


On Chip 1, the outlets for BSA and $\mathrm{BHb}$ were placed from the cathode chamber at a distance of approximately $0.465 \mathrm{~cm}$ and $0.710 \mathrm{~cm}$ respectively. On Chip 2 , the outlets for $\mathrm{BSA}$ and $\mathrm{BHb}$ were placed from the cathode chamber at a distance of approximately $0.232 \mathrm{~cm}$ and $0.355 \mathrm{~cm}$ respectively.

\subsubsection{Electrode Chamber Design}

This chamber design was based on the intent to design a clEF chamber. This design targeted the problem of the bubbles generated by the electrode chamber. In the conventional FFE device, the separation chamber is physically separated from the electrode chamber with a membrane, and previously described in Section 1.2.8. Since it is costly to incorporate such a physical membrane in a microfluidic device, Fonslow et al. used a membrane channel and an isolation buffer to mimic the membrane channel. The electrode chamber was designed to be the taller than the separation chamber to enable lubrication theory act (see Section 1.2.5.4). The electrode chamber was designed to be $20 \mu \mathrm{m}$ tall, and $2.1 \mathrm{~cm} \mathrm{~mm}$ wide for Chip1 and $1.4 \mathrm{~cm}$ wide for Chip 2. Posts were positioned throughout the electrode chamber to prevent it from collapsing.

The post geometry in the chambers were designed to be the following diameters and center-to-center distances: 

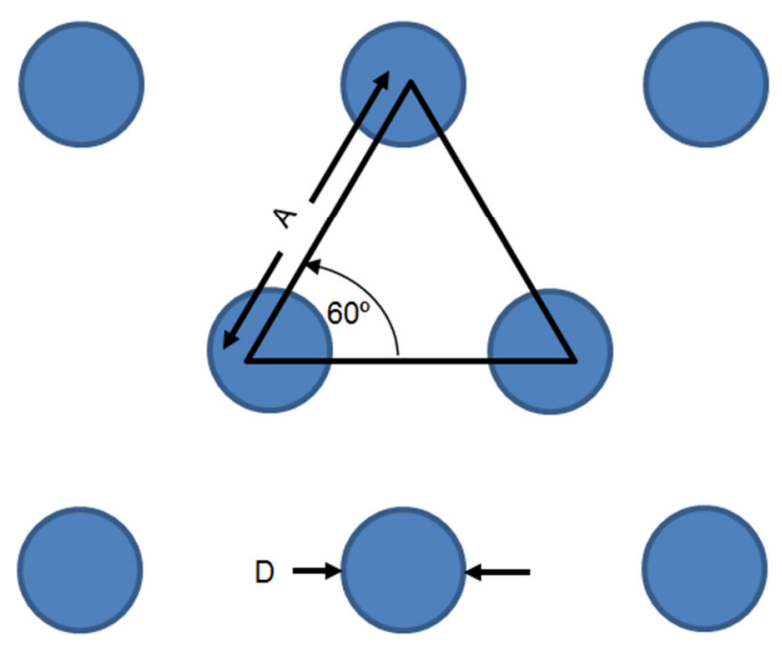

Figure 14: Microfluidic chamber post designs

Table 2: $\quad$ Post geometry specifications

\begin{tabular}{ccc}
\hline Chamber & $\mathbf{A}(\boldsymbol{\mu m})$ & Diameter $(\mu \mathrm{m})$ \\
\hline Electrode & 450 & 200 \\
Membrane & 40 & 20 \\
Separation & 135 & 50 \\
\hline
\end{tabular}

Since the electrode chamber height is much greater than its neighboring membrane channel height the higher flow rate enables the electrolysis products to be efficiently flushed out.

\subsubsection{Membrane Channel Design}

As described in Section 1.2.5.4, lubrication theory states that the difference in chamber height enables electrolysis products to be efficiently flushed out of the electrode chamber. To implement the lubrication theory, the membrane channel was designed with significantly smaller height than its neighboring channels to decrease the 
likelihood of analytes from migrating from one chamber to another. The membrane channel was designed to be the smallest in height with dimensions $1 \mathrm{~cm} \times 1 \mathrm{~mm} \times 10 \mu \mathrm{m}$ for both Chip1 and Chip2.

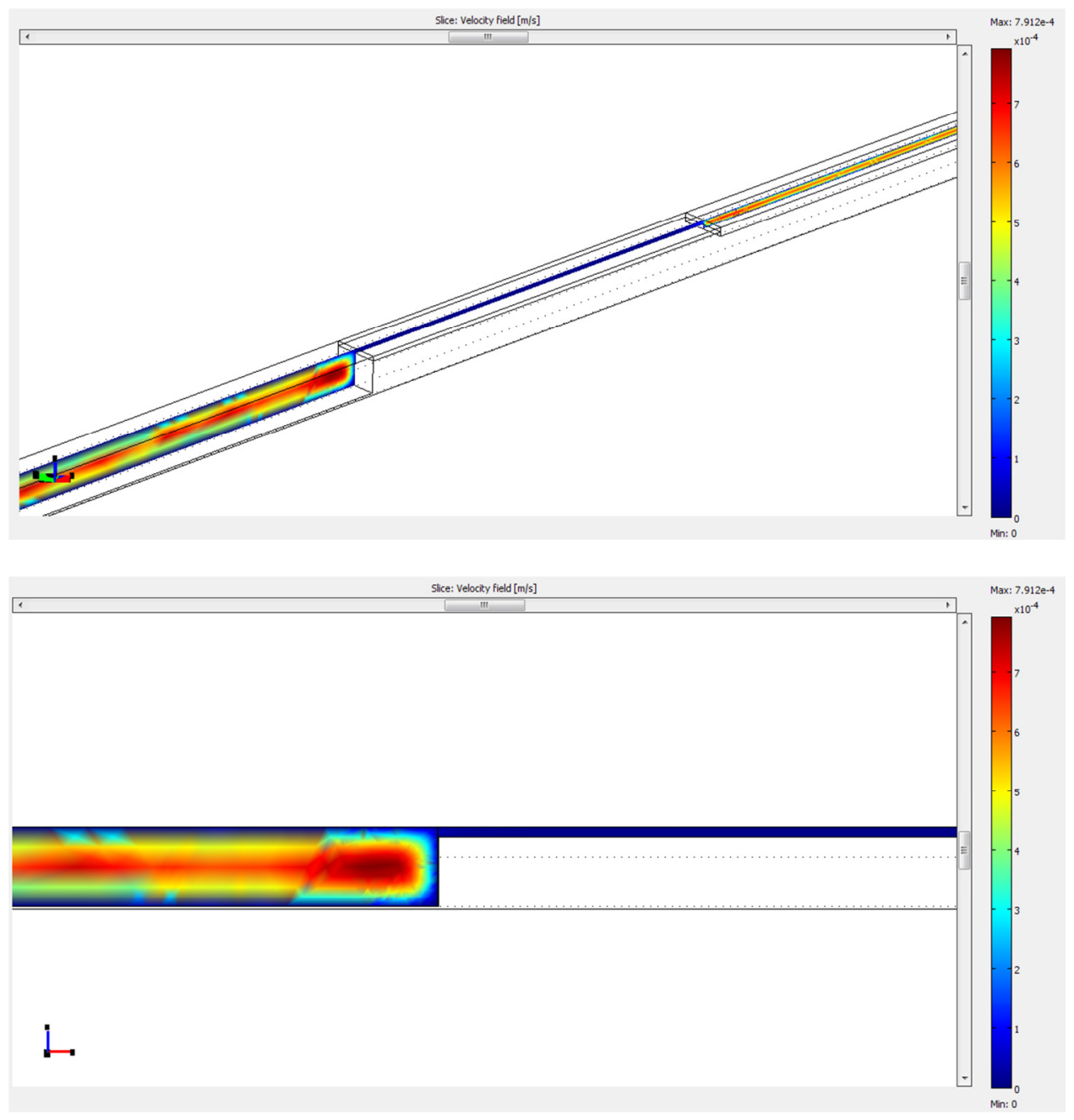

Figure 15: COMSOL modeling of velocity profile of electrode and separation chamber in overall view (above) and detailed view (below).

As seen in the above velocity gradient in the COMSOL image (Figure 15), the velocity in the electrode chamber is significantly higher than in the separation chamber.

The maximum velocities obtained from the electrode (Figure 16) and membrane chamber (Figure 17) heights are $7.3 \times 10^{-6} \mathrm{~m} / \mathrm{s}$ and $1.2 \times 10^{-6} \mathrm{~m} / \mathrm{s}$ respectively. The COMSOL results clearly delineate a decrease in velocities due to chamber heights. 


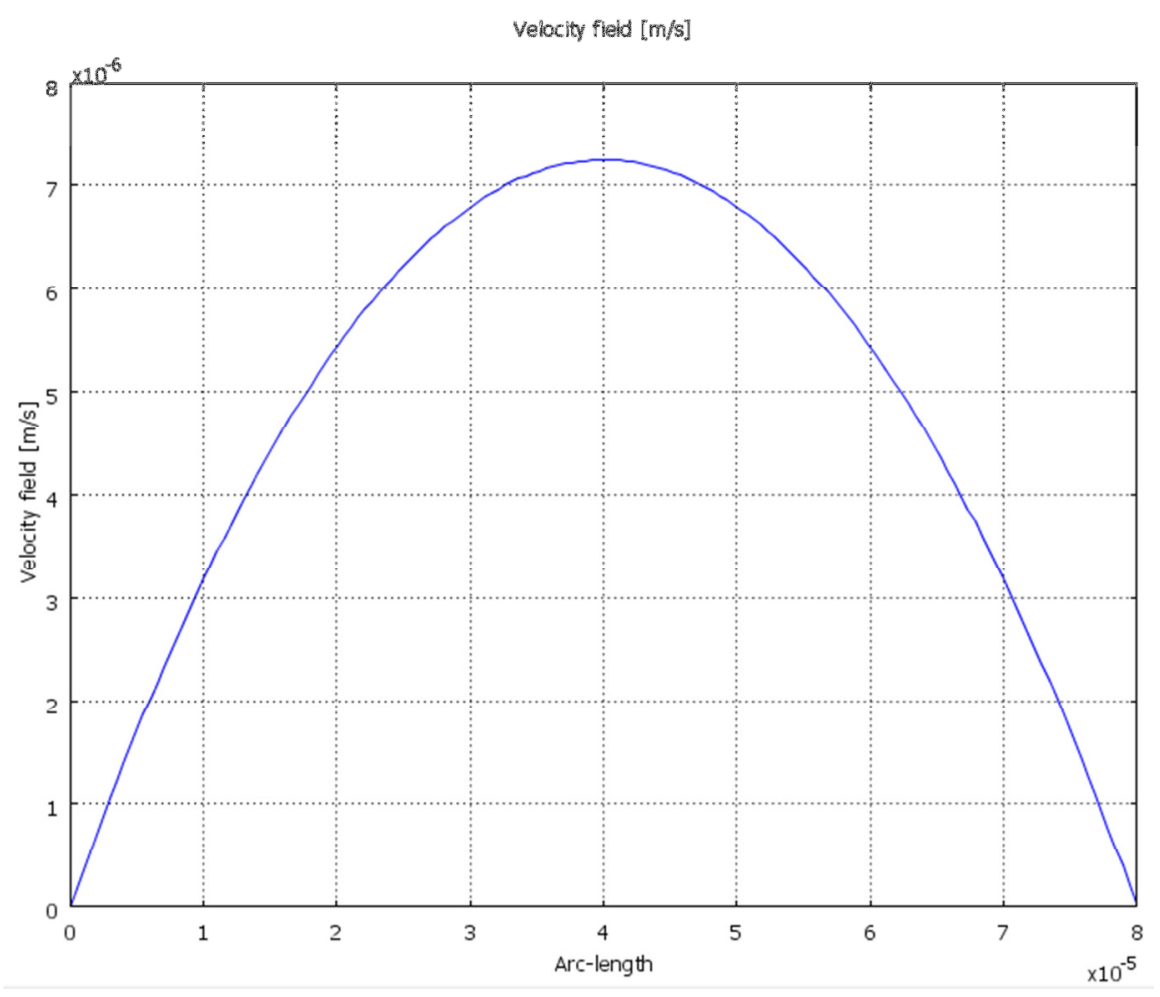

Figure 16: Velocity profile through height of the electrode chamber

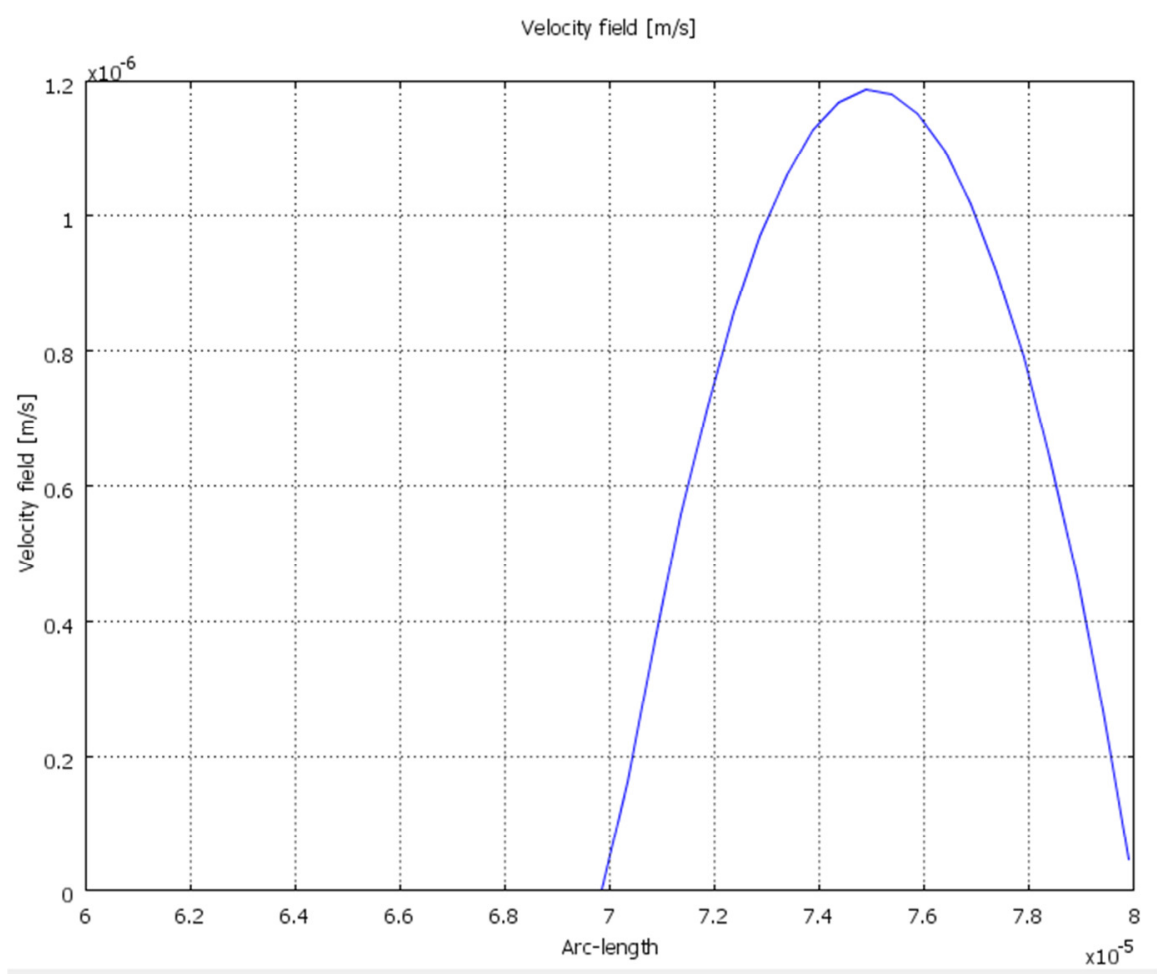

Figure 17: Velocity profile through height of the membrane chamber 
The posts were designed with the dimensions previously described in Figure 14 and Table 2.

\subsubsection{Separation Chamber Design}

The separation chamber of the chip was designed to be $1 \mathrm{~cm} \times 2.1 \mathrm{~cm} \times 20 \mu \mathrm{m}$ for Chip1 and $1 \mathrm{~cm} \times 1.4 \mathrm{~cm} \times 20 \mu \mathrm{m}$ for Chip2. The inlets to the chamber included an analyte inlet and a separate ampholyte inlet. The ampholyte inlet was designed to have a wide fan-like entrance to the separation chamber to facility dissipation in the entire chamber to form the separation medium. The analyte inlet was designed with a smaller fan-like entrance, since only a narrow stream of analytes would be flowing into the chamber. The chambers of both chips had a relatively wide area, since this would enable the ampholytes to generate a broad $\mathrm{pH}$ gradient, and possibly facilitate visualizing the separation of the analytes. Due to this wide area, posts needed to be incorporated in the design to prevent the chamber from collapsing. The posts were placed at a center-to-center distance of $60 \mu \mathrm{m}$ apart, while the post diameters were

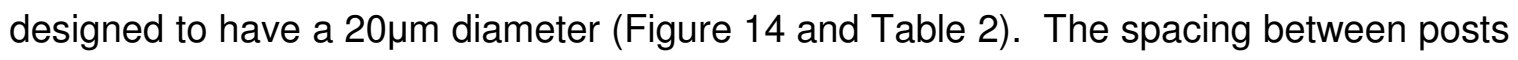
and the diameter of the posts were determined per previous chip successes and failures within the Microfluidics group.

\subsubsection{PDMS Master Mold}

An additional benefit of PDMS is that the master mold may be reused multiple times to pour a chip (Folch, Jo et al. 2000), and thus reduces costs of having to create a new master every time a chip needs to be poured. The first step of creating a master mold is by designing a template in AutoCAD, and checking the line connections using LinkCAD. This design is then used to create a high-resolution transparency that allows for UV light to pass through the specific areas as designated by the CAD file (McDonald and 
Whitesides 2002). The general fabrication method of a master mold is performed by photolithography, using a silicon wafer coated with $\mathrm{Si}_{3} \mathrm{~N}_{4}$, onto which $\mathrm{SU}$-8 photoresist is spun. The photoresist is spun onto the wafer at thicknesses corresponding to the different chamber heights of the chip, beginning with the smallest height. After each chamber height thickness of SU-8 is spun onto the chip, a high-resolution transparency mask with the chip design is placed between a UV light and the wafer. The areas exposed to the UV light are hardened and create the positive on the wafer. This is then repeated with the remaining chip chamber heights.

The Silicone wafer was large enough to fit two chip designs. To test different designs and dimensions, the following two designs were placed on the Wafer as follows: 


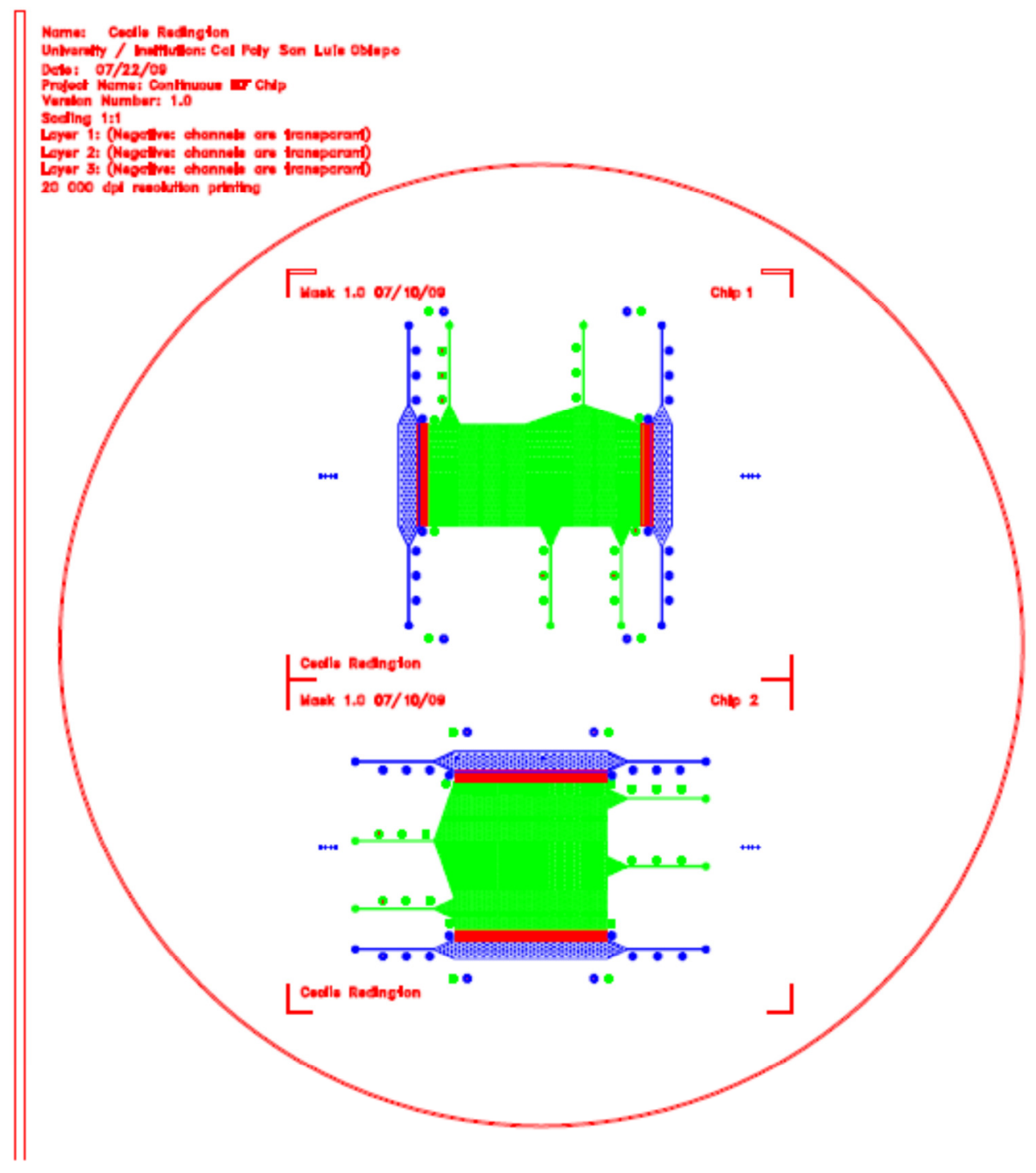

Figure 18: Placement of chip designs on wafer. 


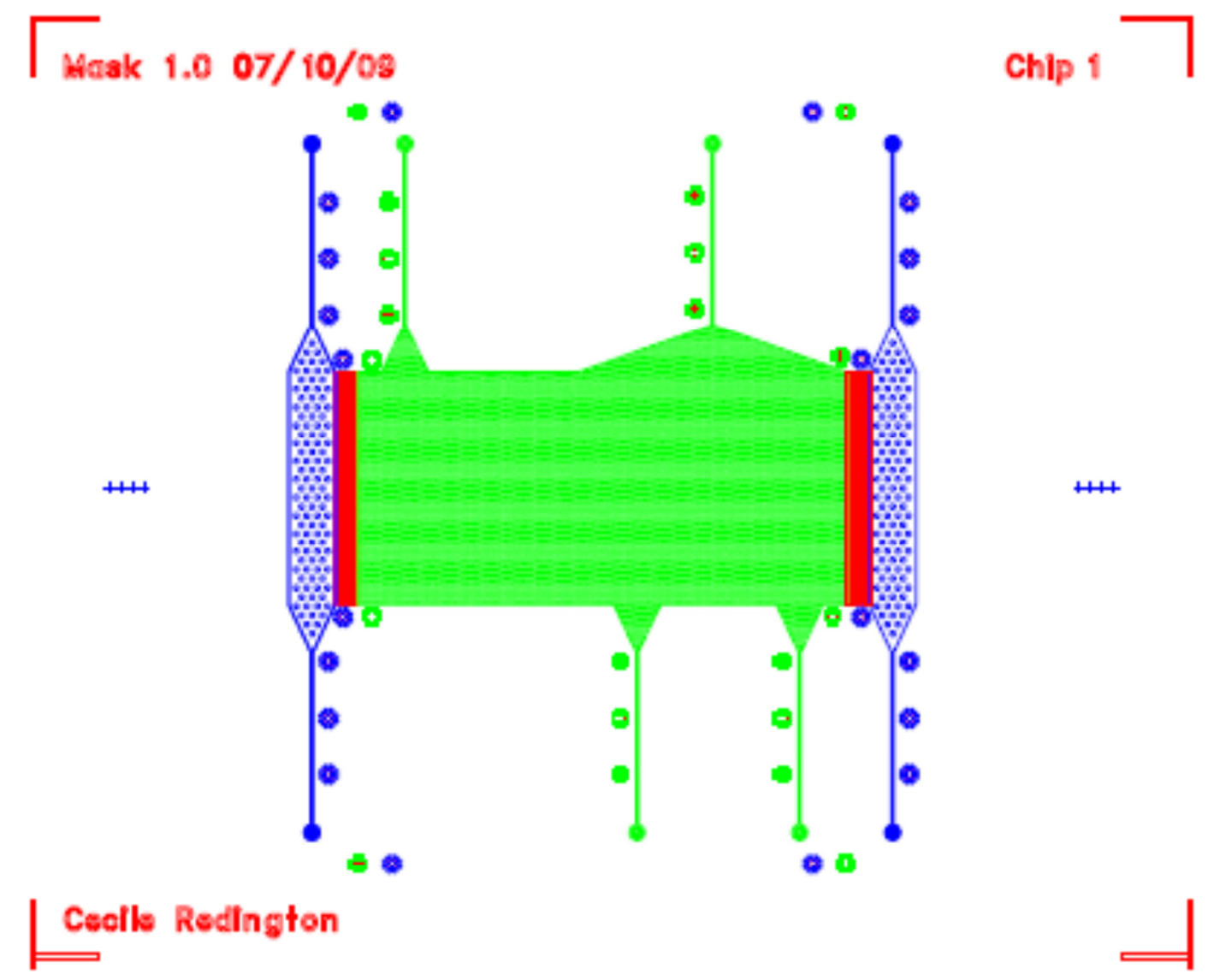

Figure 19: AutoCAD Chip 2 design 


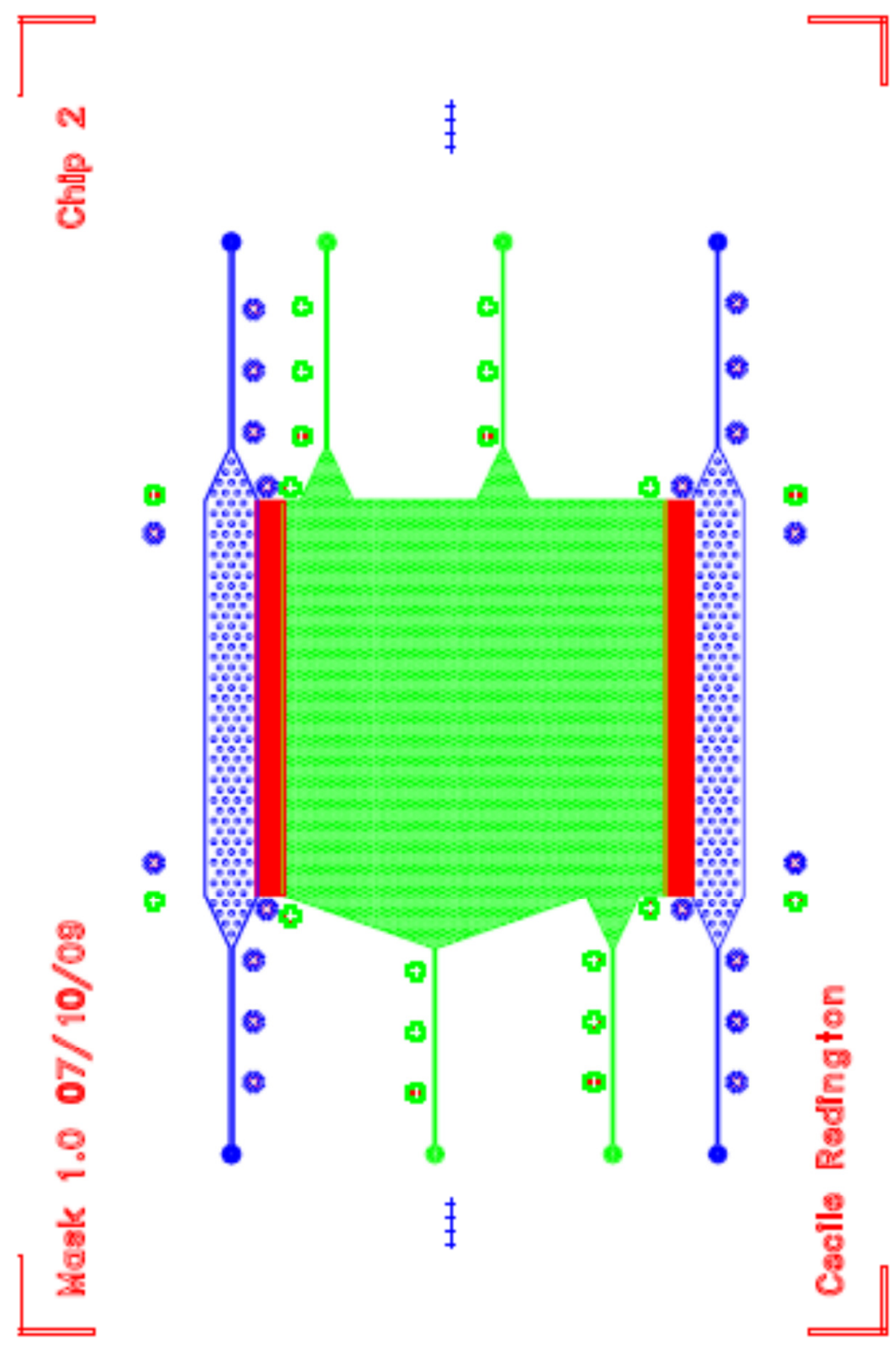

Figure 20: AutoCAD Chip 1 design

The first step in creating a PDMS chip is to create a design in AutoCAD, SolidWorks or a similar computer aided drafting program. Since the chip is designed with three different heights, each height was placed on a different layer in AutoCAD, as seen in Figure 21 and Figure 21. 


\section{Layer $3 \quad$ Layer 2}

Figure 21: Cross-sectional view of Layer organization in AutoCAD

To check if the polylines are closed in the drawings, the drawing is saved as a Gerber file, and opened in LinkCAD. This program allows to correct any discrepancies present in the drawing. The design is then printed on a transparency (photomask), allowing only the prospective chambers of the chip to be the transparent entities on the sheet. Since there are three different heights in the design, three separate photomasks needed to be printed. A silicon wafer is then spin coated with negative photoresist to create a layer with the thickness of the chamber height. The coated silicon wafer was then exposed to light through the mask, which hardened the photoresist under transparent features on the photomask. The photoresist was then rinsed off and softbaked for 10-20 minutes (Anderson, Chiu et al. 2000).

\subsubsection{Bonding}

Bonding has become an important aspect of microfluidics, and several methods have been explored by researchers. Polymer-to-polymer substrate bonding includes methods such as thermal compression, ultrasonics, and gluing using epoxy or methanol. The main consideration of sealing is deciding whether a reversible, or irreversible seal should be employed. If a reversible seal is desired, silicon or cellophane adhesive tapes can be

used. The silicon tape allows for a waterproof seal, and provides a $4^{\text {th }}$ wall composed of PDMS. The cellophane tape provides only a temporary seal. 


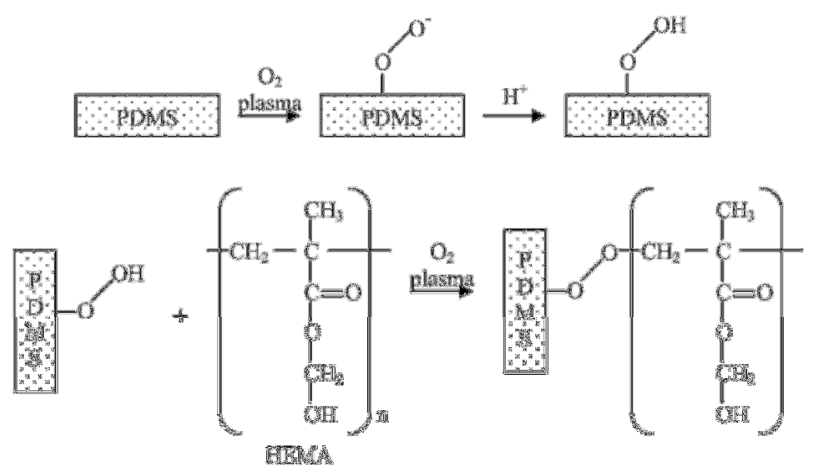

Figure 22: Oxygen plasma treatment of PDMS (Bodas and Khan Malek, 2006)

One method of generating an irreversible seal used in previous chips (Dertinger, Chiu et al. 2001) is created by exposing PDMS to air plasma. PDMS is comprised of repeated units of $-\mathrm{O}-\mathrm{Si}\left(\mathrm{CH}_{3}\right)_{2^{-}}$. By applying oxygen plasma, silanol $(-\mathrm{OH})$ groups are created on the surface of the PDMS, at the expense of methyl groups $\left(-\mathrm{CH}_{3}\right)$ (Bhattacharya, Datta et al. 2005) as seen in Figure 22. Oxidized PDMS can then effectively seal to materials that are also exposed to air plasma, such as itself, glass, silicon, polystyrene, polyethylene, and silicon nitride.

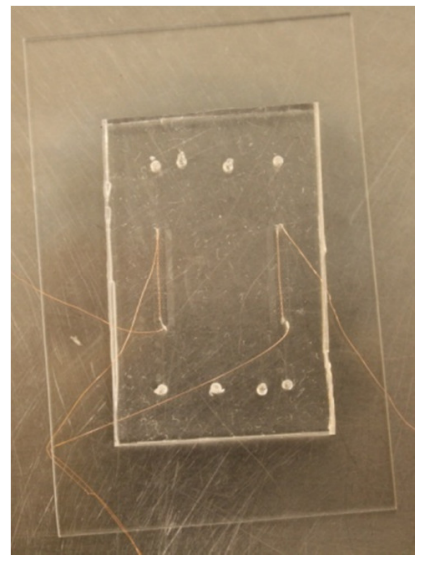

Figure 23: PDMS with copper electrodes bonded to glass slide

The sealing sides of both the PDMS and the glass slide were treated with the plasma gun. The PDMS was gently placed on the glass slide and allowed to bond to produce the bonded chip as seen in Figure 23. 


\section{EXPERIMENTAL SECTION}

Since this chip was designed for cIEF testing, the flow rates and proteins applicable for cIEF were also discussed in this section.

\subsection{Methylcellulose}

Methylcellulose (MC) is a hydrophilic, nonionic polymer, and therefore ideal to diminish the effects of electroosmotic flow (Cui, Horiuchi et al. 2005). As seen in previous studies, protein adsorption to the PDMS can hinder successful IEF (Li, DeVoe et al. 2003), since the protein does not remain in the solution, but rather adheres to the surface of the PDMS. The main approaches to conducting a successful IEF is by ideally having no EOF, or having a constant EOF (Cui, Horiuchi et al. 2005). A constant EOF would be advantageous when conducting IEF in capillary electrophoresis, since it is an effective method of pushing out the analytes during the separation process for analysis. Since this the analytes do not need to be mobilized via electrophoresis, a constant EOF is not advantageous to this experiment. Ideally, no EOF would be beneficial to conduct the current IEF experiment. A different method of maintaining the hydrophilicity of the PDMS was explored by (Cui, Horiuchi et al. 2005) introducing MC to the chip. Using the hydrophilic properties of MC, the chamber of the chip was first coated with MC, and additional MC was added to the protein-ampholyte mixture to prevent desorption of the MC from the PDMS walls (Cui, Horiuchi et al. 2005). During the IEF procedure, the EOF and the compression of the $\mathrm{pH}$ gradient were decreased, aiding in the reproducibility and resolution of the IEF (Cui, Horiuchi et al. 2005). 


\subsection{Food Dye}

Food dye was chosen for the analysis of EOF in the microfluidic chip, and Green McCormick's Food Dye was purchased from the grocery store. Food dye is an inexpensive and very effective method of visualizing the experiment carried out in the microfluidic chip. Food dye is readily available, cost-effective, and non-toxic. No special material handling is required to use this analyte. The main ingredients of this analyte are: Water, Propylene Glycol, FD\&C Yellow 5, FD\&C Blue 1, and Propylparaben. Food dye was used in the chip to analyze the EO velocity.

\subsection{Flow Rates}

The flow rates in the chip were calculated based on the lubrication theory (Fonslow, Barocas et al. 2006). Assuming that the flow rate (q) in the electrode chambers will be 16 times greater than in the separation chamber. Applying Fonslow's equation of the flow rate:

$$
\frac{q_{\text {separation }}}{q_{\text {electrode }}}=\frac{A_{\text {separation }} \cdot v_{\text {separation }}}{A_{\text {electrode }} \cdot v_{\text {electrode }}}=\frac{1}{16}
$$

First, the cross sectional areas (A) of the chambers were calculated to be roughly 1.6 $\times 10^{-7} \mathrm{~m}^{2}$ and $4.2 \times 10^{-7} \mathrm{~m}^{2}$ for the electrode and separation chamber respectively. By running dye through the separation chamber at different flow rates, it was seen that the highest rate was at $0.5 \mathrm{ml} / \mathrm{min}$ without damaging any components of the chip. The velocity is therefore: 


$$
\begin{aligned}
v_{\text {separation }}= & \frac{q_{\text {separation }}}{A_{\text {separation }}}=\frac{0.01 \frac{\mathrm{ml}}{\mathrm{min}}}{4.2 \times 10^{-7} \mathrm{~m}^{2}} \cdot \frac{1}{1000 \mathrm{ml}} \cdot \frac{\mathrm{m}^{3}}{1000 \mathrm{l}} \cdot \frac{60 \mathrm{~s}}{1 \mathrm{~min}} \quad \text { Equation } 22 \\
& =1.19 \frac{\mathrm{m}}{\mathrm{min}}
\end{aligned}
$$

Substituting these values into the previous equation of the flow rate ratio, the velocity of each electrode chamber was determined to be $49.98 \mathrm{~m} / \mathrm{min}$.

$$
\begin{gathered}
v_{\text {electrode }}=16 \cdot \frac{A_{\text {separation }} v_{\text {separation }}}{A_{\text {electrode }}} \\
=16 \cdot \frac{4.2 \times 10^{-7} \mathrm{~m}^{2} \cdot 1.19 \frac{\mathrm{m}}{\mathrm{min}}}{1.6 \times 10^{-7} \mathrm{~m}^{2} 1}=49.98 \frac{\mathrm{m}}{\mathrm{min}}
\end{gathered}
$$

Equation 23

\subsection{Reagents}

The following reagents in the given concentration and molarity were purchased from their respective companies: $\mathrm{NaOH}$ (Science Company, Denver, CO), $7 \mathrm{mM} \mathrm{H}_{3} \mathrm{PO}_{4}, 400$ cP 2\% Methylcellulose (Sigma-Aldrich, St. Louis, MO), Bio-Lyte 3/10 Ampholyte (Invitrogen, Carlsbad, CA), Bovine Hemoglobin (USB Corporation, Cleveland, Ohio), Bovine Serum Albumin (Invitrogen, Carlsbad, CA), 10x IEF Anode Buffer (Invitrogen, Carlsbad, CA)

Since the reagents were purchased at the available set concentration, some needed to be diluted to fit the chip. To dilute the available reagents, the following equation was applied:

$$
M_{\text {initial }} V_{\text {initial }}=M_{\text {finial }} V_{\text {final }}
$$

Where $\mathrm{M}$ represents the molarity, and $\mathrm{V}$ the volume of the reagent. Using Equation 21 , it was possible to dilute the reagents to the following molarities, and create the necessary solutions within the listed in the protocols. 


\subsection{Equipment Set-Up}

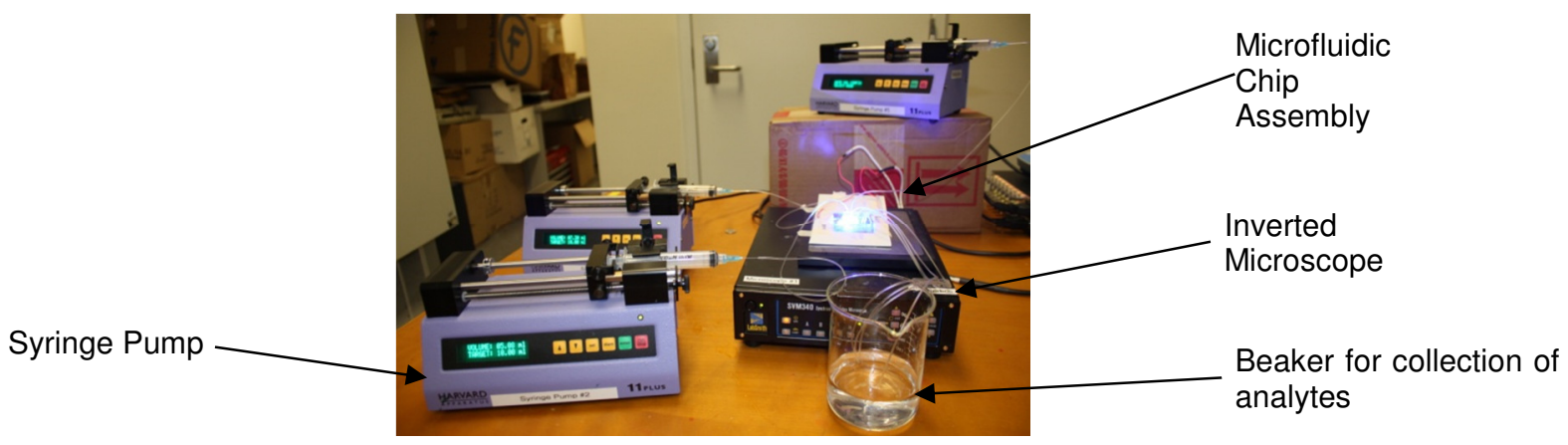

Figure 24: Overall Experimental Set-up.

The chip was set up as seen in Figure 24, with the 40 gauge electrode wires coming from the PDMS chip connected to 20 gauge wires leading to the voltage supply. Tygon tubing was inserted directly into the PDMS chip to serve as electrode and analyte channel inlets and outlets. The tygon tubing leading to the inlets were connected to syringes, while the tubing located at the outlets were allowed to flow into a beaker.

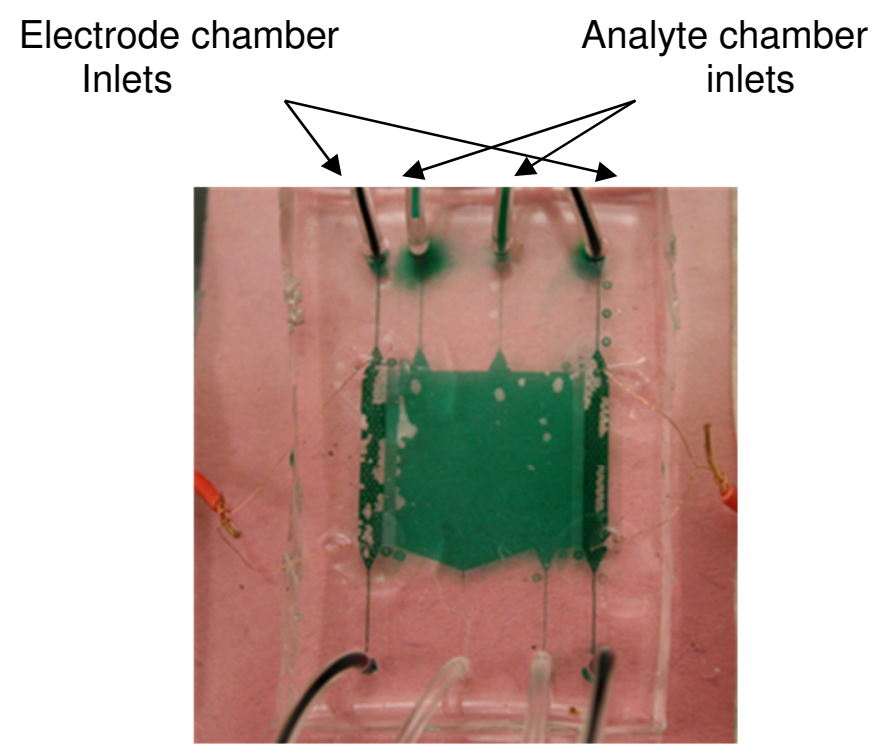

Figure 25: Chamber inlets 


\subsection{Sealing and Leakage Testing}

To test the seal of the chip, isopropyl alcohol was run through the chambers of the chip. This particular fluid was used because it's quick evaporation time, leaving the chip dry and enabling experimentation with different sealants if necessary. The air plasma bonding of the chip to the glass was effectively carried out. The locations at which the electrode wires entered the chamber unfortunately enabled the fluid to escape the chamber.

Several attempts were made of applying silicone window and door sealant to these locations, and allowed to dry for 1,2, 4, and 7 days. After each drying time, isopropyl alcohol was run through the chip to test fluid leakage. None of these drying times were successful, and the silicone remained soft and didn't adhere to the PDMS, allowing the fluid to leak out of the electrode wire locations.

The next attempt to prevent the leakage was pour PDMS at the leaking locations. PDMS was mixed in the Microfabrication Lab at Cal Poly with a 10:1 (base:curing agent) ratio. The PDMS was allowed to degas in a vacuum chamber for approximately 20 minutes until all the bubbles were removed from the liquid. A drop of PDMS was then placed on each electrode wire inlet, and the chip was subsequently cured in an oven at $75^{\circ} \mathrm{C}$ for 40 minutes. After running isopropyl alcohol through the chip, it was apparent that the sealing was successfully carried out. 


\section{RESULTS}

\subsection{Chip Fabrication}

The silicon wafers and initial two chips were commissioned and fabricated at the Stanford Microfluidics Lab.

\subsubsection{PDMS chip fabrication}

The PDMS chip was poured per the protocol delineated in Joshua Fadriquela's Thesis project (see Section E in the Appendix). The fabrication of PDMS was performed by forming a 10:1 mixture of PDMS prepolymer and curing agent. Air bubbles were formed and entrapped in the mixture during the stirring, which are problematic for creating a microfluidic chip, since these would create flaws in the chip. These air bubbles were removed by placing the mixture in a vacuum chamber for approximately 20 minutes or until no bubbles were present in the mixture. The silicon wafer is placed in a tinfoil-lined petri dish, and the PDMS is carefully poured onto the wafer, trying to not infuse additional air bubbles. The tinfoil with the PDMS and wafer are then placed in an

oven at $65{ }^{\circ} \mathrm{C}$ for approximately 40 minutes. After baking, the hardened PDMS is carefully peeled from the wafer, and the area with the design is carefully trimmed using a razor knife.

\subsubsection{Electrodes}

As seen in previous chips, such as the one designed by Macanova et al., (Macounova, Cabrera et al. 2000),

To minimize cost and to step away from the conventional method of using printed electrodes, 40 gauge copper wire. To strive for a simple and cost-effective design, 
electrode wires were 'sewn' into the chip as seen in Figure 47 before it was bonded to the glass slide.

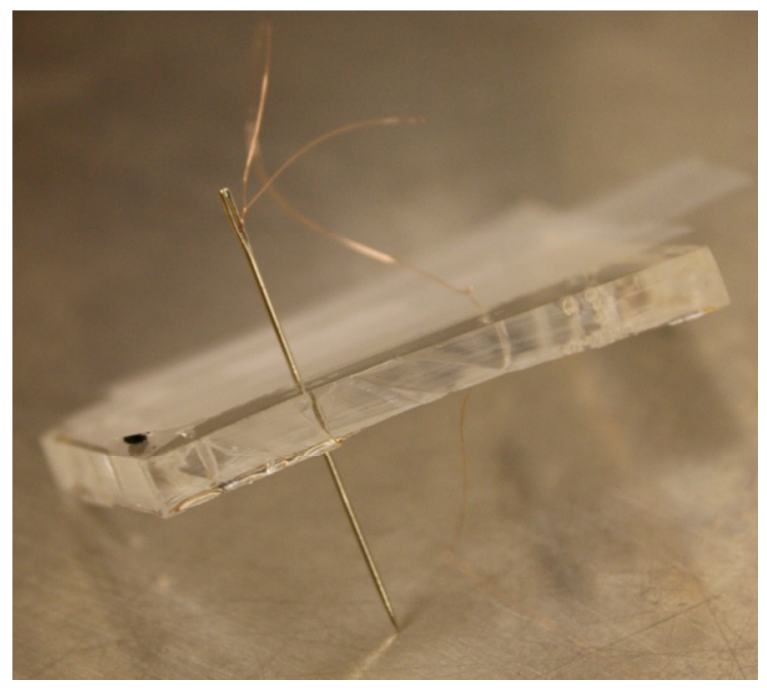

Figure 26: Electrode "sewing" with 40 gauge wire.

Electrode sewing was performed in the clean room to prevent dust from being incorporated in the chip's chamber. The initial step when handling the PDMS chip during which it is exposed to the air for an extended period of time is to place some tape lightly onto the side of the PDMS where the features were created by the silicone wafer.

The needle was only inserted on the feature-side of the PDMS, since it is inevitable to prevent PDMS from tearing and causing particulation during the sewing. Therefore, the particulates are pushed to the exit location of the needle. If inserting the needle on the non-featured side of the PDMS, particulates would be created on the featured-side, which are eventually trapped in the chambers once the PDMS is bonded to the glass.

A thin stitching needle was threaded with the copper wire, and inserted through the tape, into the chamber of the PDMS between the two analyte ports, approximately $1 \mathrm{~mm}$ from where the electrode chamber analyte inlet design fans to the electrode chamber. The needle was then pulled through the PDMS until there was approximately 6" slack. The needle was then reinserted in the PDMS approximately $1 \mathrm{~mm}$ from where the 
design of the electrode chamber converges to the analyte outlet. The needle was reinserted at this location in the PDMS and pulled through. This same process was subsequently repeated at the cathodic electrode chamber.

Each copper wire was then separately soldered to an insulated 20 gauge wire, which was eventually connected to the power supply.

\subsection{Chip Experiments}

The analysis was carried out using two different methods: by using the inverted microscope and by taking pictures with subsequent analysis in ImageJ. The inverted microscope method was carried out first, and yielded velocities between $913.7 \mu \mathrm{m} / \mathrm{sec}$ and $948.95 \mu \mathrm{m} / \mathrm{sec}$ for an untreated chip (Section 4.3), whereas the COMSOL model yielded a maximum velocity of $0.00025 \mu \mathrm{m} / \mathrm{sec}$ (Section 4.6). Due to this discrepancy, the velocity was also measured by taking pictures of the chip and computing the flow velocity by analyzing the quantity of food dye in the chip using ImageJ (Section 0), which yielded results between $2.567 \mu \mathrm{m} / \mathrm{s}$ and $812.9 \mu \mathrm{m} / \mathrm{s}$.

\subsection{Via Inverted Microscope}

Multiple test runs were performed with each treatment and all raw data can be found in the Appendix. The results with the highest $p$-values that conform to a normal distribution were chosen to be representative of each treatment and discussed in the following paragraphs.

\subsubsection{Analysis with $\mathrm{NaOH}$ treatment at $25 \mathrm{~V} / \mathrm{cm}$}

After analyzing all test results from this treatment, the following Test 5 of the test runs was determined to be the most appropriate batch, due to its high $p$-value at 
$\mathrm{p}=0.314(\mathrm{p}>0.05)$, indicating a normal distribution. This test treatment was conducted with the application of $\mathrm{NaOH}$ at $25 \mathrm{~V} / \mathrm{cm}$ (see protocol of Section $\mathrm{K}$ ).

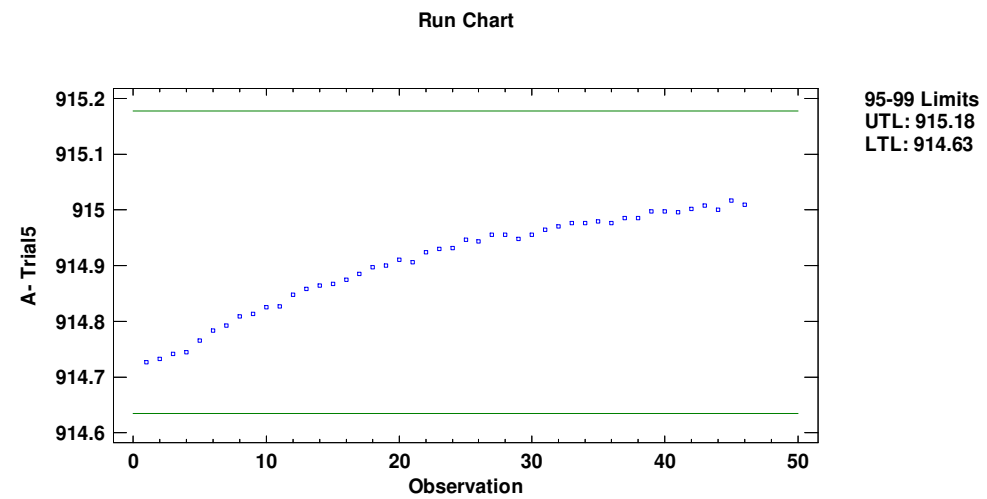

Goodness-of-Fit Tests for A- Trial5

Kolmogorov-Smirnov Test

\begin{tabular}{|l|l|}
\hline & Normal Distribution \\
\hline DPLUS & 0.100201 \\
\hline DMINUS & 0.141899 \\
\hline DN & 0.141899 \\
\hline P-value & 0.31431 \\
\hline
\end{tabular}

The StatAdvisor

This pane shows the results of tests run to determine whether A- Trial5 can be adequately modeled by a Normal distribution. The Kolmogorov-Smirnov test computes the maximum distance between the cumulative distribution of A- Trial5 and the CDF of the fitted Normal distribution. In this case, the maximum distance is 0.141899.

Since the smallest P-value amongst the tests performed is greater than or equal to 0.05 , we can not reject the idea that A- Trial5 comes from a Normal with $95 \%$ confidence.

Summary Statistics for A- Trial5

\begin{tabular}{|l|l|}
\hline Count & 46 \\
\hline Average & 914.906 \\
\hline Standard deviation & 0.0861921 \\
\hline Coeff. of variation & $0.00942086 \%$ \\
\hline Minimum & 914.726 \\
\hline Maximum & 915.017 \\
\hline Range & 0.290937 \\
\hline Stnd. skewness & -1.87183 \\
\hline Stnd. kurtosis & -0.980469 \\
\hline
\end{tabular}

Figure 27: StatGraphics results of testing with $\mathrm{NaOH}$ treatment at $25 \mathrm{~V} / \mathrm{cm}$

\subsubsection{Analysis with $\mathrm{NaOH}$ treatment at $50 \mathrm{~V} / \mathrm{cm}$}

After analyzing all test results from this treatment, the following Test 2 of the test runs was determined to be the most appropriate batch, due to its high $p$-value at 
$p=0.303(p>0.05)$, indicating a normal distribution. This test treatment was conducted with the application of $\mathrm{NaOH}$ at $50 \mathrm{~V} / \mathrm{cm}$ (see protocol listed in Section K).

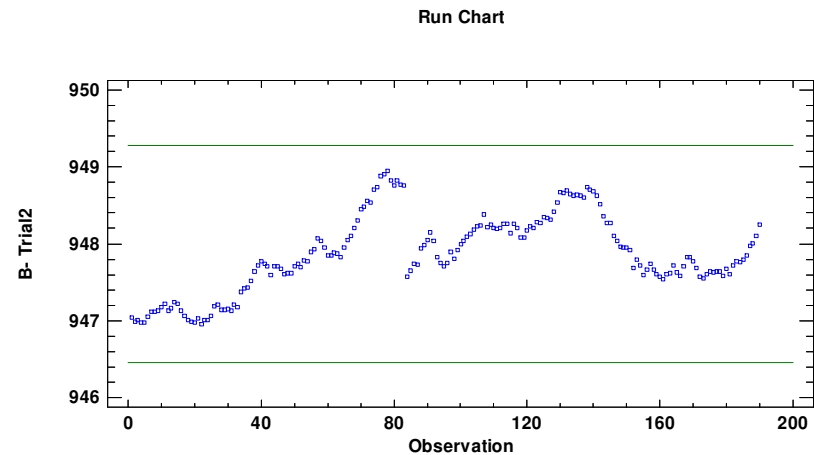

\section{5-99 Limits}

\section{Goodness-of-Fit Tests for B- Trial2}

Kolmogorov-Smirnov Test

\begin{tabular}{|l|l|}
\hline & Normal Distribution \\
\hline DPLUS & 0.0704903 \\
\hline DMINUS & 0.0699043 \\
\hline DN & 0.0704903 \\
\hline P-value & 0.30322 \\
\hline
\end{tabular}

The StatAdvisor

This pane shows the results of tests run to determine whether B- Trial2 can be adequately modeled by a Normal distribution. The Kolmogorov-Smirnov test computes the maximum distance between the cumulative distribution of B- Trial2 and the CDF of the fitted Normal distribution. In this case, the maximum distance is 0.0704903 .

Since the smallest P-value amongst the tests performed is greater than or equal to 0.05 , we can not reject the idea that B- Trial2 comes from a Normal with $95 \%$ confidence.

Summary Statistics for B- Trial2

\begin{tabular}{|l|l|}
\hline Count & 190 \\
\hline Average & 947.869 \\
\hline Standard deviation & 0.501556 \\
\hline Coeff. of variation & $0.0529141 \%$ \\
\hline Minimum & 946.953 \\
\hline Maximum & 948.95 \\
\hline Range & 1.99667 \\
\hline Stnd. skewness & 0.330978 \\
\hline Stnd. kurtosis & -1.77123 \\
\hline
\end{tabular}

Figure 28: StatGraphics results of testing with $\mathrm{NaOH}$ treatment at $50 \mathrm{~V} / \mathrm{cm}$

\subsubsection{Analysis with $\mathrm{NaOH}$ treatment at $75 \mathrm{~V} / \mathrm{cm}$}

After analyzing all test results from this treatment, the following Test 1 of the test runs was determined to be the most appropriate batch, due to its high $p$-value at 
$p=0.721 \quad(p>0.05)$, indicating a normal distribution. This test treatment was conducted with the application of $\mathrm{NaOH}$ at $75 \mathrm{~V} / \mathrm{cm}$ (see protocol listed in Section $\mathrm{K}$ ).

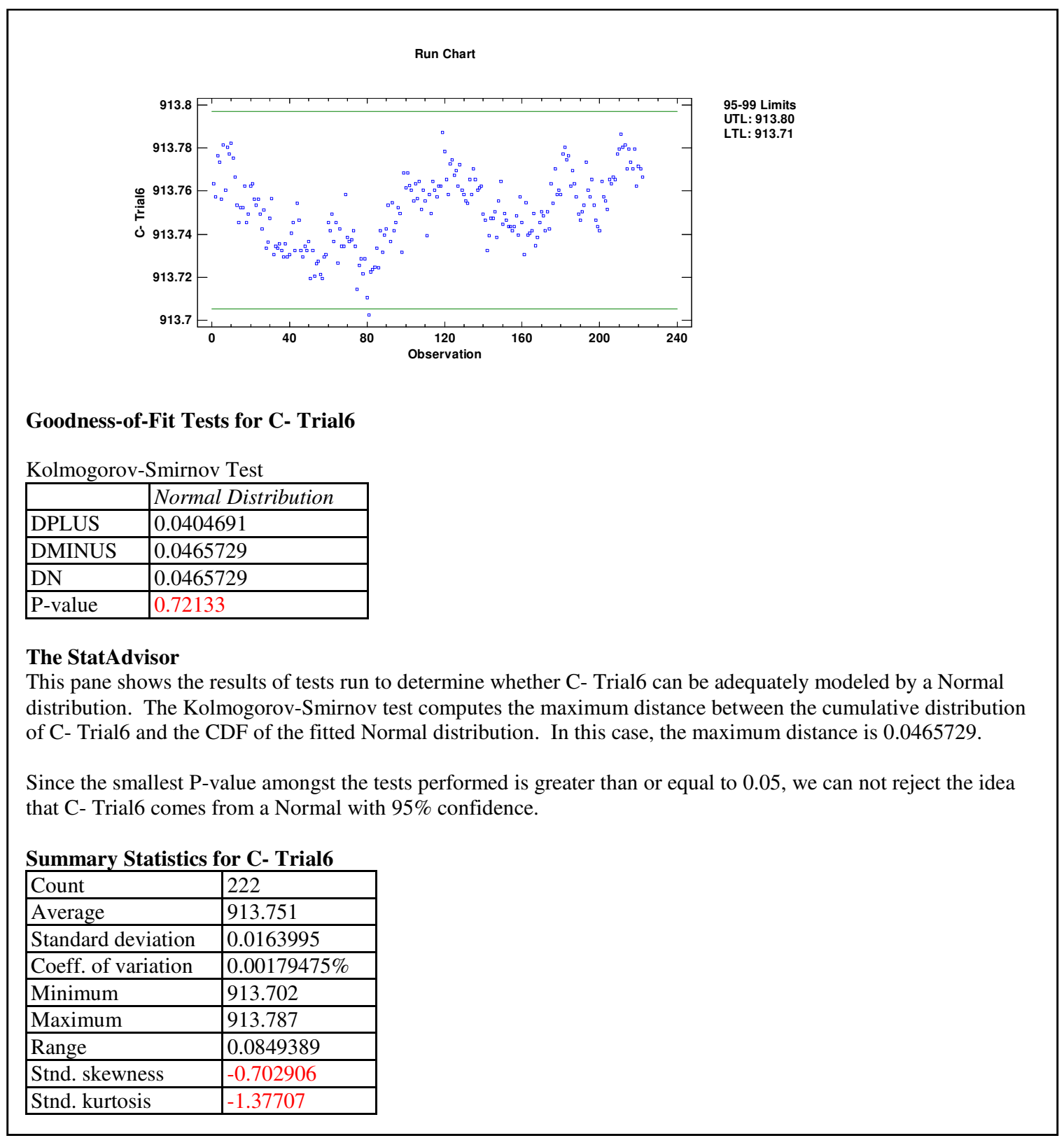

Figure 29: StatGraphics results of testing with $\mathrm{NaOH}$ treatment at $75 \mathrm{~V} / \mathrm{cm}$

\subsubsection{Analysis with $\mathrm{NaOH}$ and $\mathrm{MC}$ treatment at $25 \mathrm{~V} / \mathrm{cm}$}

After analyzing all test results from this treatment, the following Test 1 of the test runs was determined to be the most appropriate batch, due to its high $p$-value at 
$\mathrm{p}=0.058(\mathrm{p}>0.05)$, indicating a normal distribution. This test treatment was conducted without the application of $\mathrm{NaOH}$ and $\mathrm{MC}$ at $75 \mathrm{~V} / \mathrm{cm}$ (see protocol listed in Section $\mathrm{L}$ ).

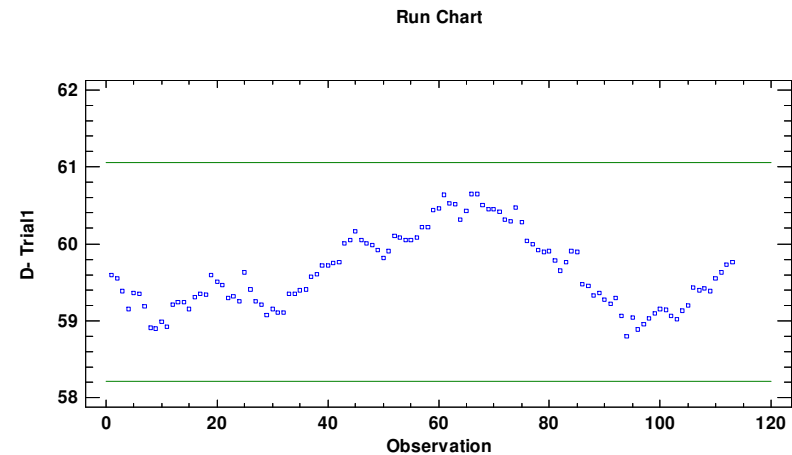

95-99 Limits

UTL: 61.06
LTL: 58.21

Goodness-of-Fit Tests for D- Trial1

Kolmogorov-Smirnov Test

\begin{tabular}{|l|l|}
\hline & Normal Distribution \\
\hline DPLUS & 0.12495 \\
\hline DMINUS & 0.0607032 \\
\hline DN & 0.12495 \\
\hline P-value & 0.0587031 \\
\hline
\end{tabular}

The StatAdvisor

This pane shows the results of tests run to determine whether D- Trial1 can be adequately modeled by a Normal distribution. The Kolmogorov-Smirnov test computes the maximum distance between the cumulative distribution of D- Trial1 and the CDF of the fitted Normal distribution. In this case, the maximum distance is 0.12495.

Since the smallest P-value amongst the tests performed is greater than or equal to 0.05 , we can not reject the idea that D- Trial1 comes from a Normal with $95 \%$ confidence.

Summary Statistics for D- Trial1

\begin{tabular}{|l|l|}
\hline Count & 113 \\
\hline Average & 59.634 \\
\hline Standard deviation & 0.488751 \\
\hline Coeff. of variation & $0.819585 \%$ \\
\hline Minimum & 58.796 \\
\hline Maximum & 60.653 \\
\hline Range & 1.857 \\
\hline Stnd. skewness & 1.83855 \\
\hline Stnd. kurtosis & -1.95734 \\
\hline
\end{tabular}

Figure 30: StatGraphics results of testing with $M C$ treatment at $25 \mathrm{~V} / \mathrm{cm}$ 


\subsection{Via Pictures and ImageJ analysis}

All testing was carried out by treating the chip per the applicable protocols, then subsequently filling the chip with food die as seen in Figure 31 below.

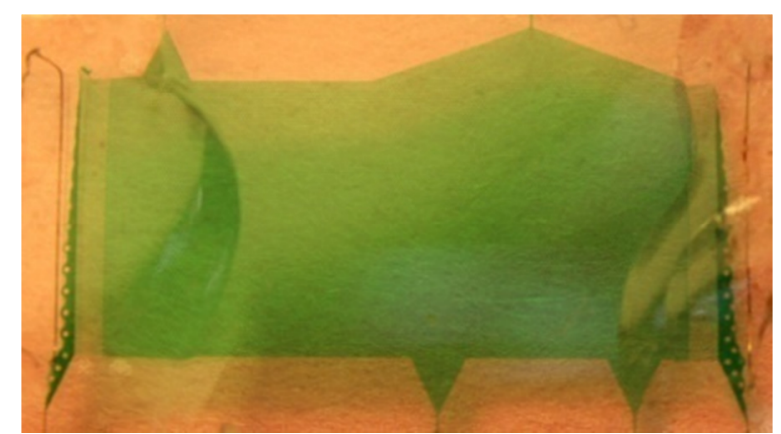

Figure 31: Initial food die content

After the fluid was observed to no longer flow, a voltage was applied, and it the fluid moved toward the anode of the chip, as seen below in Figure 32.

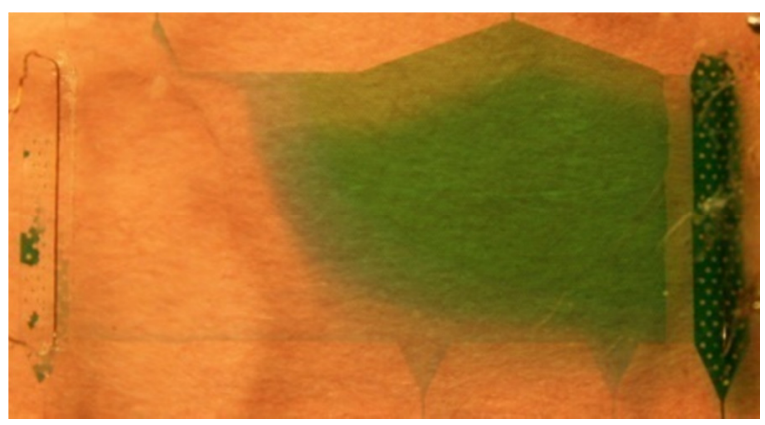

Figure 32: Dye displacement after 5 minutes.

At certain time intervals a picture of the chip was taken. For the analysis of the data, the image was converted to a black and white image, and cropped to show only the separation chamber as seen below in Figure 33.

Figure 33: Image preparation for analysis In ImageJ. 
The image was then analyzed in ImageJ, by converting it to an 8-bit image as shown in Figure 34, and the summary of measurements of the black areas containing dye were taken as seen in Figure 35.

Figure 34: Image conversion into 8-bit in ImageJ.

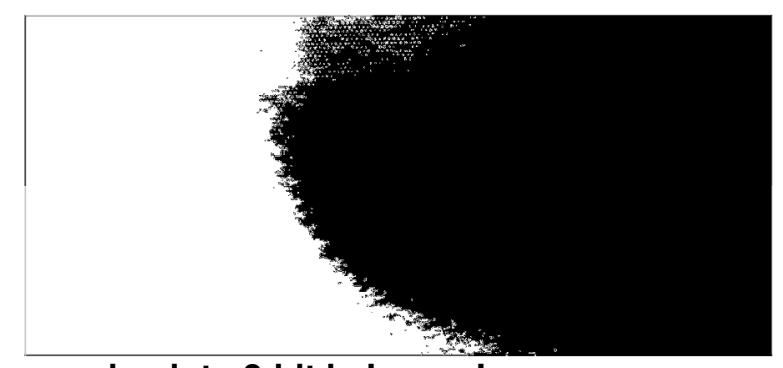

\begin{tabular}{|l|l|l|l|l|l|l|}
\hline File Edit & Font & - & \\
\hline Slice & Count & Total Area & Average Size & \%Area & Mean & \\
\hline samples.jpg & 104 & $1.081 \mathrm{E}-4$ & $1.039 \mathrm{E}-6$ & 59.535 & 255 & \\
& & & & & & \\
\hline
\end{tabular}

Figure 35: ImageJ area measurement summary.

To determine the flow velocity, the areas of dye were converted to volumes. Based on the change in volume taken at the individual time increments, the flow velocity was determined.

\subsubsection{Analysis with $\mathrm{NaOH}$ treatment at $25 \mathrm{~V} / \mathrm{cm}$}

To establish that EOF does exist in the chip, food dye was run through the chip. All inlets and outlets worked perfectly and the chip was in good condition. The chip was first flushed with DI water then dye was inserted in both inlets of the separation chamber. While the dye was running into the separation chamber, the DI water was inserted in the electrode chamber inlets, until the electrode chambers were roughly clear of green food dye. The procedure was performed at $25 \mathrm{~V} / \mathrm{cm}$ by the protocol (Section $\mathrm{K}$ ) in the Appendix. This first test was carried out in a $2 \mathrm{~cm}$ wide chip, and recorded at $5 \mathrm{~min}$ increments. It was quickly seen that 5 min increments in the initial part of the testing was too long to document results. It was also confirmed that EOF does exist in the chip, 
once the dye was introduced, which would pose a problem in future testing. After $60 \mathrm{~min}$, the dye was confined to the anodic electrode chamber.

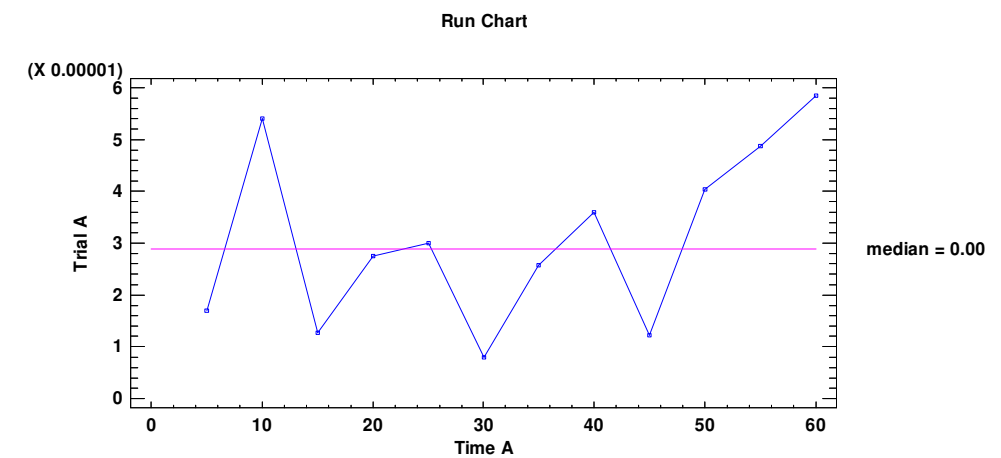

Goodness-of-Fit Tests for Trial A

Kolmogorov-Smirnov Test

\begin{tabular}{|l|l|}
\hline & Normal Distribution \\
\hline DPLUS & 0.12794 \\
\hline DMINUS & 0.104406 \\
\hline DN & 0.12794 \\
\hline P-value & 0.989413 \\
\hline
\end{tabular}

\section{The StatAdvisor}

This pane shows the results of tests run to determine whether Trial A can be adequately modeled by a Normal distribution. The Kolmogorov-Smirnov test computes the maximum distance between the cumulative distribution of Trial A and the CDF of the fitted Normal distribution. In this case, the maximum distance is 0.12794.

Since the smallest P-value amongst the tests performed is greater than or equal to 0.05 , we can not reject the idea that Trial A comes from a Normal with $95 \%$ confidence.

\section{Summary Statistics for Trial A}

\begin{tabular}{|l|l|}
\hline Count & 12 \\
\hline Average & 0.0000308967 \\
\hline Standard deviation & 0.0000169806 \\
\hline Coeff. of variation & $54.9594 \%$ \\
\hline Minimum & 0.00000791 \\
\hline Maximum & 0.00005853 \\
\hline Range & 0.00005062 \\
\hline Stnd. skewness & 0.396803 \\
\hline Stnd. kurtosis & -0.807276 \\
\hline
\end{tabular}

Figure 36: StatGraphics results of testing with $\mathrm{NaOH}$ treatment at $25 \mathrm{~V} / \mathrm{cm}$ 


\subsubsection{Analysis with $\mathrm{NaOH}$ treatment at $50 \mathrm{~V} / \mathrm{cm}$}

The procedure was performed with the same methods as the previous experiment (Section 4.4.1), but was conducted at $50 \mathrm{~V} / \mathrm{cm}$ by the protocol (Section $\mathrm{K}$ ) in the Appendix.

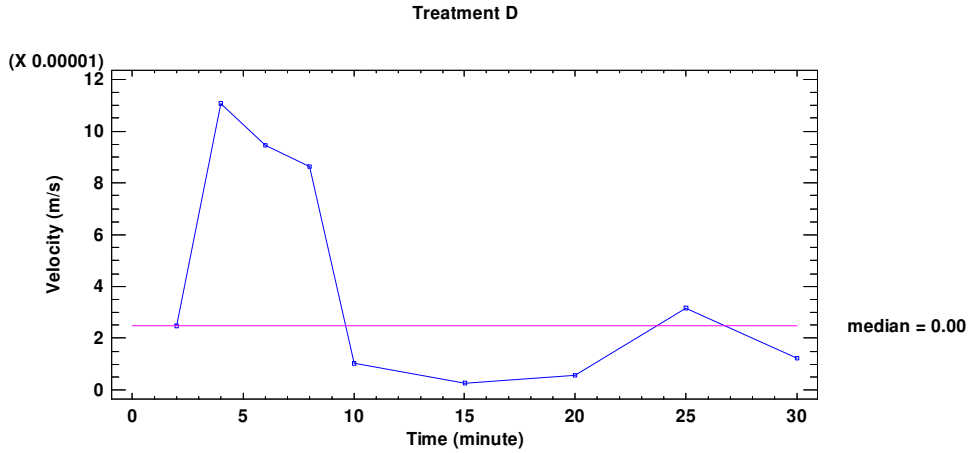

Goodness-of-Fit Tests for Trial D

Kolmogorov-Smirnov Test

\begin{tabular}{|l|l|}
\hline & Normal Distribution \\
\hline DPLUS & 0.261778 \\
\hline DMINUS & 0.182206 \\
\hline DN & 0.261778 \\
\hline P-value & 0.568178 \\
\hline
\end{tabular}

The StatAdvisor

This pane shows the results of tests run to determine whether Trial D can be adequately modeled by a Normal distribution. The Kolmogorov-Smirnov test computes the maximum distance between the cumulative distribution of Trial D and the CDF of the fitted Normal distribution. In this case, the maximum distance is 0.261778.

Since the smallest $\mathrm{P}$-value amongst the tests performed is greater than or equal to 0.05 , we can not reject the idea that Trial D comes from a Normal with $95 \%$ confidence.

Summary Statistics for Trial D

\begin{tabular}{ll}
\hline Count & 9
\end{tabular}

\begin{tabular}{|l|l|}
\hline Average & 0.0000421181 \\
\hline
\end{tabular}

\begin{tabular}{|l|l|}
\hline Standard deviation & 0.000042741 \\
\hline
\end{tabular}

\begin{tabular}{|l|l}
\hline Coeff. of variation & $101.479 \%$ \\
\hline
\end{tabular}

\begin{tabular}{|l|l|}
\hline Minimum & 0.000002707 \\
\hline
\end{tabular}

\begin{tabular}{|l|l}
\hline Maximum & 0.0001107 \\
\hline
\end{tabular}

\begin{tabular}{|l|l|}
\hline Range & 0.000107993 \\
\hline
\end{tabular}

\begin{tabular}{|l|l|}
\hline Stnd. skewness & 0.955211 \\
\hline
\end{tabular}

\begin{tabular}{|l|l|}
\hline Stnd. kurtosis & -0.849594 \\
\hline
\end{tabular}

\section{Figure 37: StatGraphics results of testing with $\mathrm{NaOH}$ treatment at $50 \mathrm{~V} / \mathrm{cm}$}




\subsubsection{Analysis with $\mathrm{NaOH}$ treatment at $75 \mathrm{~V} / \mathrm{cm}$}

The next test was performed by first rinsing the chambers with $1 \mathrm{M} \mathrm{NaOH}$ to deprotonate its surfaces, followed by a MC rinse to suppress EOF. The procedure was performed at $75 \mathrm{~V} / \mathrm{cm}$ by the protocol (Section $\mathrm{K}$ ) in the Appendix.

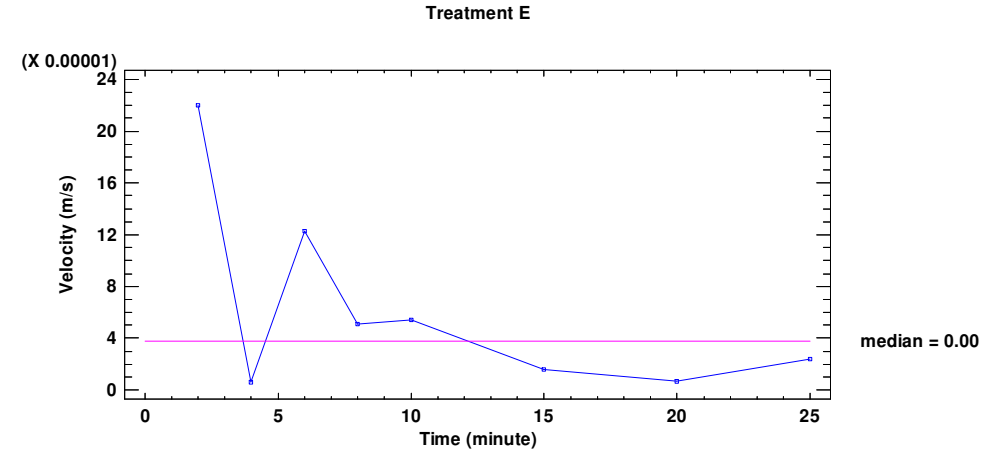

Goodness-of-Fit Tests for Trial E

Kolmogorov-Smirnov Test

\begin{tabular}{|l|l|}
\hline & Normal Distribution \\
\hline DPLUS & 0.296255 \\
\hline DMINUS & 0.222875 \\
\hline DN & 0.296255 \\
\hline P-value & 0.494725 \\
\hline
\end{tabular}

The StatAdvisor

This pane shows the results of tests run to determine whether Trial E can be adequately modeled by a Normal distribution. The Kolmogorov-Smirnov test computes the maximum distance between the cumulative distribution of Trial E and the CDF of the fitted Normal distribution. In this case, the maximum distance is 0.296255.

Since the smallest P-value amongst the tests performed is greater than or equal to 0.05 , we can not reject the idea that Trial E comes from a Normal with $95 \%$ confidence.

\section{Summary Statistics for Trial E}

\begin{tabular}{|l|l|}
\hline Count & 8 \\
\hline Average & 0.0000625796 \\
\hline Standard deviation & 0.0000742668 \\
\hline Coeff. of variation & $118.676 \%$ \\
\hline Minimum & 0.00000595 \\
\hline Maximum & 0.0002201 \\
\hline Range & 0.00021415 \\
\hline Stnd. skewness & 1.93841 \\
\hline Stnd. kurtosis & 1.43712 \\
\hline
\end{tabular}

Figure 38: StatGraphics results of testing with $\mathrm{NaOH}$ treatment at $75 \mathrm{~V} / \mathrm{cm}$ 


\subsubsection{Analysis with $\mathrm{NaOH}$ and $\mathrm{MC}$ treatment at $25 \mathrm{~V} / \mathrm{cm}$}

The procedure was performed by treating rinsing the chip with $\mathrm{NaOH}$ as described in Section 4.4.1, then treating the chip with MC. This experiment was performed at 25 $\mathrm{V} / \mathrm{cm}$ by the protocol (Section L)

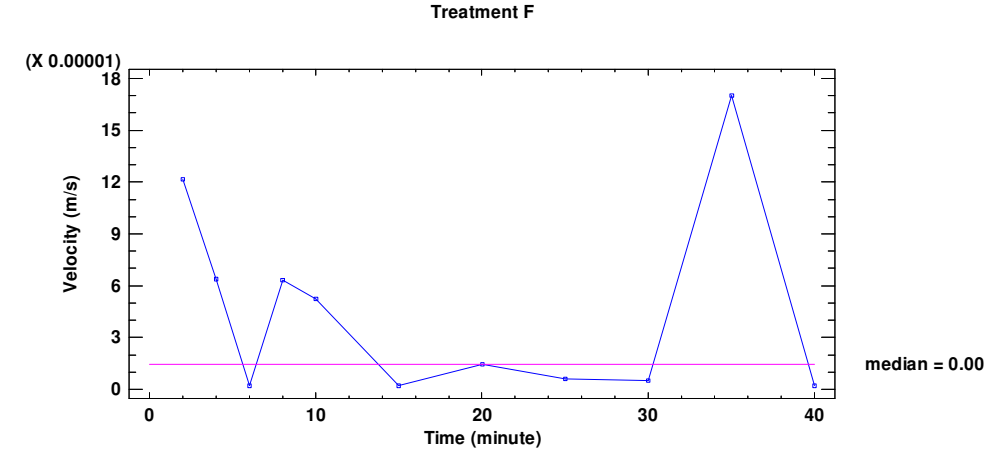

\section{Goodness-of-Fit Tests for Trial F}

Kolmogorov-Smirnov Test

\begin{tabular}{|l|l|}
\hline & Normal Distribution \\
\hline DPLUS & 0.254956 \\
\hline DMINUS & 0.218756 \\
\hline DN & 0.254956 \\
\hline P-value & 0.481865 \\
\hline
\end{tabular}

\section{The StatAdvisor}

This pane shows the results of tests run to determine whether Trial $\mathrm{F}$ can be adequately modeled by a Normal distribution. The Kolmogorov-Smirnov test computes the maximum distance between the cumulative distribution of Trial $\mathrm{F}$ and the CDF of the fitted Normal distribution. In this case, the maximum distance is 0.254956 .

Since the smallest P-value amongst the tests performed is greater than or equal to 0.05 , we can not reject the idea that Trial F comes from a Normal with $95 \%$ confidence.

\section{Summary Statistics for Trial $\mathbf{F}$}

\begin{tabular}{|l|l|}
\hline Count & 11 \\
\hline Average & 0.0000457129 \\
\hline Standard deviation & 0.0000564078 \\
\hline Coeff. of variation & $123.396 \%$ \\
\hline Minimum & 0.000001918 \\
\hline Maximum & 0.0001701 \\
\hline Range & 0.000168182 \\
\hline Stnd. skewness & 1.80643 \\
\hline Stnd. kurtosis & 0.728665 \\
\hline
\end{tabular}

Figure 39: StatGraphics results of testing with $\mathrm{NaOH}$ and $\mathrm{MC}$ treatment at $25 \mathrm{~V} / \mathrm{cm}$ 


\subsection{Statistical Summary}

\subsubsection{Statistical Analysis of Inverted Microscope results}

Table 3: Summary of test results per the Inverted Microscope

\begin{tabular}{lllll}
\hline & $\mathrm{NaOH}$ & $\mathrm{NaOH}$ & $\mathrm{NaOH}$ & $\mathrm{NaOH}+\mathrm{MC}$ \\
& $25 \mathrm{~V} / \mathrm{cm}$ & $50 \mathrm{~V} / \mathrm{cm}$ & $75 \mathrm{~V} / \mathrm{cm}$ & $25 \mathrm{~V} / \mathrm{cm}$ \\
\hline Count & 46 & 190 & 222 & 113 \\
Average $(\boldsymbol{\mu m} / \mathbf{s})$ & 914.906 & 947.869 & 913.751 & 59.634 \\
Standard deviation & 0.0861921 & 0.501556 & 0.0163995 & 0.488751 \\
Coeff. of variation & $0.00942086 \%$ & $0.0529141 \%$ & $0.00179475 \%$ & $0.819585 \%$ \\
Minimum $(\boldsymbol{\mu m} / \mathbf{s})$ & 914.726 & 946.953 & 913.702 & 58.796 \\
Maximum $(\boldsymbol{\mu m} / \mathbf{s})$ & 915.017 & 948.95 & 913.787 & 60.653 \\
Range & 0.290937 & 1.99667 & 0.0849389 & 1.857 \\
Stnd. skewness & -1.87183 & 0.330978 & -0.702906 & 1.83855 \\
Stnd. kurtosis & -0.980469 & -1.77123 & -1.37707 & -1.95734 \\
Normality $(\mathbf{p}-\mathbf{v a l u e})$ & 0.31431 & 0.30322 & 0.72133 & 0.0587031 \\
\hline
\end{tabular}

The velocity at $25 \mathrm{~V} / \mathrm{cm}$ of the untreated versus the $\mathrm{MC}$ treated chip decreases by the following ratio:

$$
\frac{v_{\text {no treatment }}}{v_{\text {treatment with } M C}}=\frac{914.906 \mu \mathrm{m} / \mathrm{s}}{59.634 \mu \mathrm{m} / \mathrm{s}}=\mathbf{1 5 . 3 4 2}
$$

\begin{tabular}{|l|l|l|l|l|}
\hline \multicolumn{5}{|c|}{} \\
IM- NaOH, 50V/cm \\
IM- NaOH, 75V/cm \\
IM- NaOH+Mc, 25V/cm
\end{tabular}




\begin{tabular}{|l|l|l|l|l|}
\hline Maximum & 915.017 & 948.95 & 913.786 & 60.653 \\
\hline Range & 0.290937 & 1.88829 & 0.0449723 & 1.857 \\
\hline Stnd. skewness & -1.87183 & 1.44567 & -0.078818 & 1.83855 \\
\hline Stnd. kurtosis & -0.980469 & -1.17168 & -1.45477 & -1.95734 \\
\hline
\end{tabular}

\section{ANOVA Table}

\begin{tabular}{|l|l|l|l|l|l|}
\hline Source & Sum of Squares & Df & Mean Square & F-Ratio & P-Value \\
\hline Between groups & $6.08864 \mathrm{E} 7$ & 3 & $2.02955 \mathrm{E} 7$ & 133669387.25 & 0.0000 \\
\hline Within groups & 56.9375 & 375 & 0.151833 & & \\
\hline Total (Corr.) & $6.08865 \mathrm{E} 7$ & 378 & & & \\
\hline
\end{tabular}

\section{The StatAdvisor}

The ANOVA table decomposes the variance of the data into two components: a between-group component and a within-group component. The F-ratio, which in this case equals $1.33669 \mathrm{E} 8$, is a ratio of the between-group estimate to the within-group estimate. Since the P-value of the F-test is less than 0.05 , there is a statistically significant difference between the means of the 4 variables at the $95.0 \%$ confidence level. To determine which means are significantly different from which others, select Multiple Range Tests from the list of Tabular Options.

Figure 40: ANOVA Inverted Microscope results of $\mathrm{NaOH}$ (at 25, 50, and 75V/cm) and $\mathrm{NaOH}+\mathrm{MC}($ at $25 \mathrm{~V} / \mathrm{cm})$ treatments.

By analyzing the box-and-whisker plot of Figure 40 , the $\mathrm{NaOH}+\mathrm{MC}$ results were significantly lower than the treatment using $\mathrm{NaOH}$ only. Since $\mathrm{p}<0.05$, the means of the different treatments are statistically not the same. Therefore, the treatments have an effect on the experiment.

To statistically analyze if the different potentials applied to the chip have an effect on the flow velocities, ANOVA was performed on the data of treatments performed only with $\mathrm{NaOH}:$ 


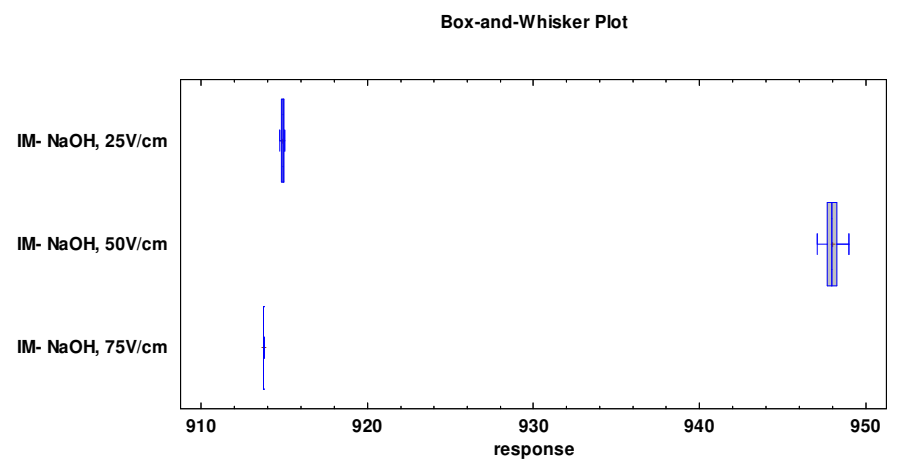

ANOVA Table

\begin{tabular}{|l|l|l|l|l|l|}
\hline Source & Sum of Squares & $D f$ & Mean Square & F-Ratio & P-Value \\
\hline Between groups & 70886.7 & 2 & 35443.3 & 308833.94 & 0.0000 \\
\hline Within groups & 30.1832 & 263 & 0.114765 & & \\
\hline Total (Corr.) & 70916.9 & 265 & & & \\
\hline
\end{tabular}

The StatAdvisor

The ANOVA table decomposes the variance of the data into two components: a between-group component and a within-group component. The F-ratio, which in this case equals 308834., is a ratio of the between-group estimate to the within-group estimate. Since the P-value of the Ftest is less than 0.05 , there is a statistically significant difference between the means of the 3 variables at the $95.0 \%$ confidence level. To determine which means are significantly different from which others. select Multiple Range Tests from the list of Tabular Ontions.

Figure 41: Statgraphics analysis per Inverted Microscope- Results of $\mathrm{NaOH}$ only treatment (at 25,50 , and $75 \mathrm{~V} / \mathrm{cm})$.

\subsubsection{Statistical Analysis of Pictures and ImageJ results}

Table 4: Summary of test results per Pictures and ImageJ

\begin{tabular}{lllll}
\hline & $\mathrm{NaOH}$ & $\mathrm{NaOH}$ & $\mathrm{NaOH}$ & $\mathrm{NaOH}+\mathrm{MC}$ \\
& $25 \mathrm{~V} / \mathrm{cm}$ & $50 \mathrm{~V} / \mathrm{cm}$ & $75 \mathrm{~V} / \mathrm{cm}$ & $25 \mathrm{~V} / \mathrm{cm}$ \\
\hline Count & 12 & 9 & 8 & 11 \\
Average & 0.0000308967 & 0.0000421181 & 0.0000625796 & 0.0000457129 \\
Standard deviation & 0.0000169806 & 0.000042741 & 0.0000742668 & 0.0000564078 \\
Coeff. of variation & $54.9594 \%$ & $101.479 \%$ & $118.676 \%$ & $123.396 \%$ \\
Minimum & 0.00000791 & 0.000002707 & 0.00000595 & 0.000001918 \\
Maximum & 0.00005853 & 0.0001107 & 0.0002201 & 0.0001701 \\
Range & 0.00005062 & 0.000107993 & 0.00021415 & 0.000168182 \\
Stnd. skewness & 0.396803 & 0.955211 & 1.93841 & 1.80643 \\
Stnd. kurtosis & -0.807276 & -0.849594 & 1.43712 & 0.728665 \\
Normality (p-value) & 0.989413 & 0.568178 & 0.494725 & 0.481865 \\
\hline
\end{tabular}




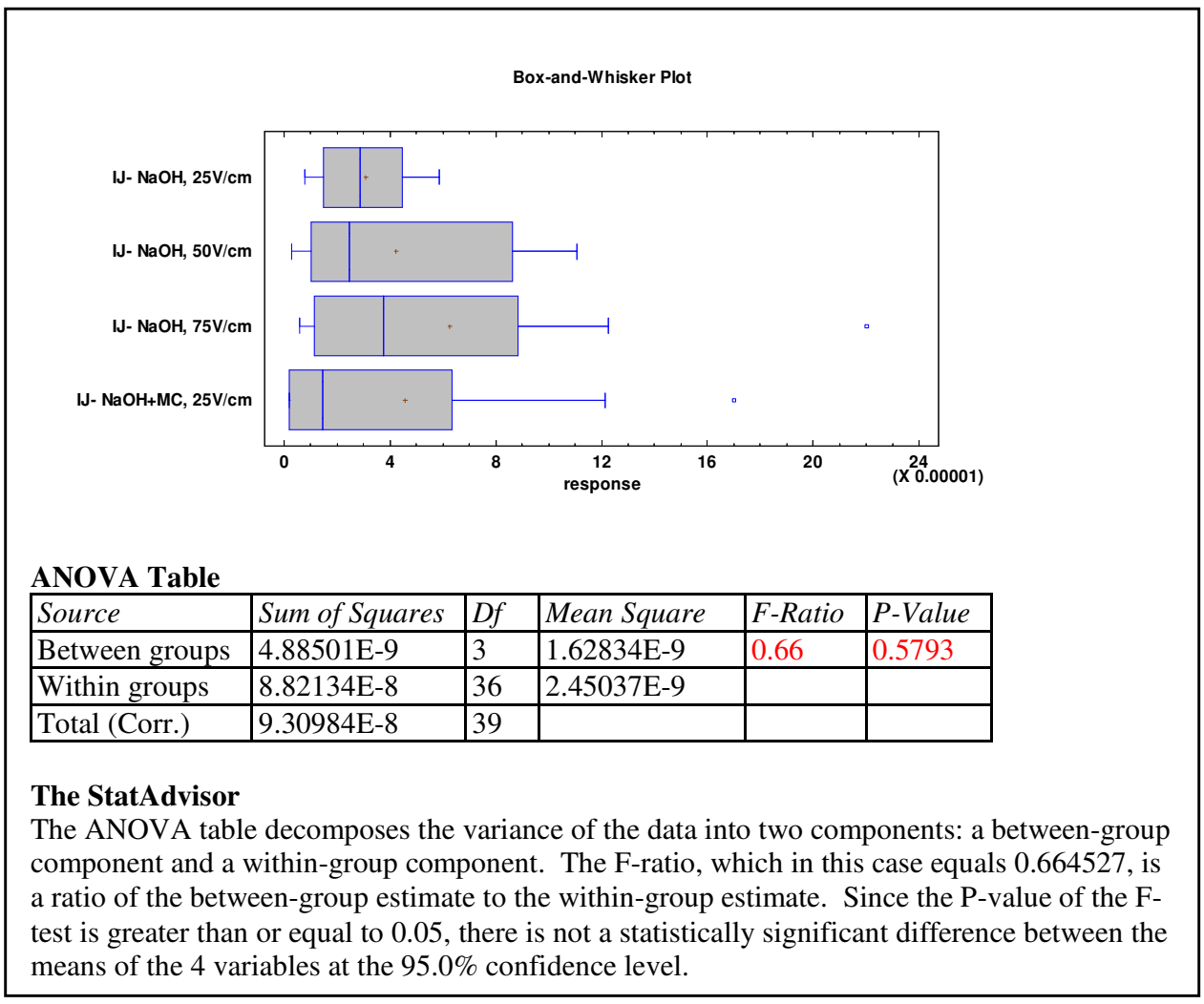

Figure 42: ANOVA ImageJ results of $\mathrm{NaOH}$ (at 25, 50 , and $75 \mathrm{~V} / \mathrm{cm}$ ) and $\mathrm{NaOH}+\mathrm{MC}$ (at $25 \mathrm{~V} / \mathrm{cm}$ ) treatments.

\subsection{COMSOL}

\subsubsection{Lubrication Theory}

A partial 3D model was generated to analyze lubrication theory:

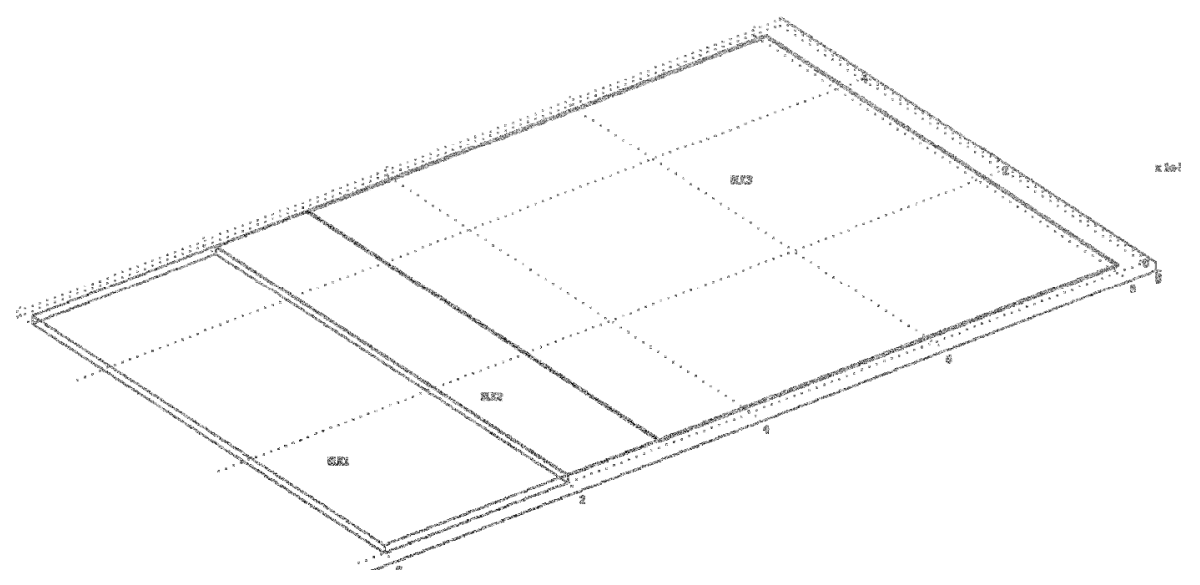

Figure 43: COMSOL 3D model for lubrication theory analysis. 
To analyze the difference in flow velocities between the electrode chamber and the membrane channel, the cross section was analyzed:

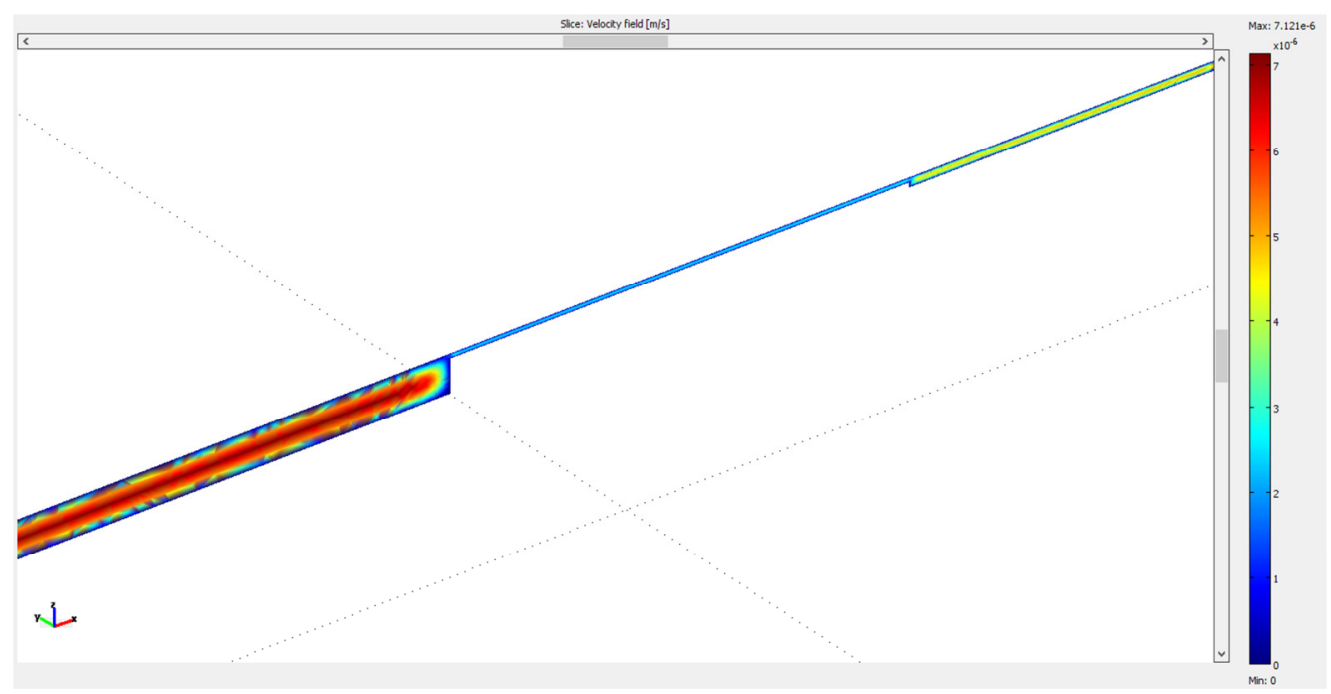

Figure 44: Cross-sectional slice of flow velocity

The velocity profile generated at the center of the electrode chamber through the height axis:
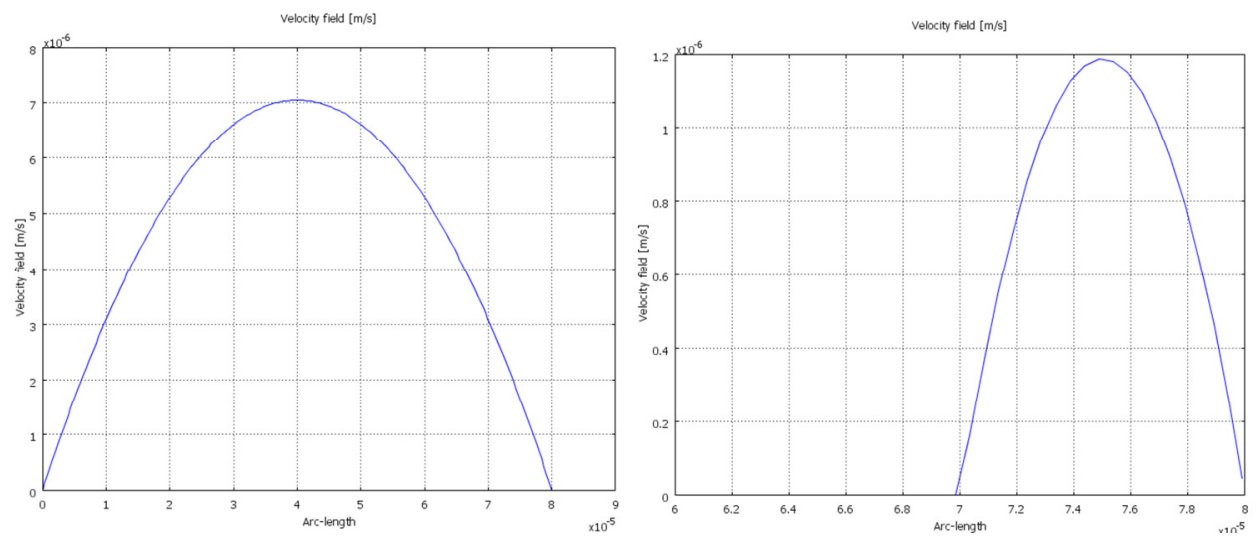

Figure 45: Flow velocity in the electrode (left) and membrane (right) chambers.

Velocity of the profile at the center of the membrane channel through the height axis:

The flow velocity ratio per COMSOL model:

$$
\frac{\text { electrode chamber velocity }}{\text { membrane channel velocity }}=\frac{7.3 \times 10^{-6} \frac{\mathrm{m}}{\mathrm{s}}}{1.2 \times 10^{-6} \frac{\mathrm{m}}{\mathrm{s}}}=\mathbf{6 . 1}
$$

Per the COMSOL model, the ratio of the velocities is 6.1 . 


\subsubsection{EOF}

The COMSOL model was generated to model the untreated chip, and to analyze the flow velocities when only DI water is contained in the chip. The cross section of the chip was designed in COMSOL with the exact dimensions of the chip, and the following electrical conditions applied to the model:

Table 5: COMSOL Parameters to analyze EOF.

\begin{tabular}{ll}
\hline Parameter & Value \\
\hline Voltage & $25 \mathrm{~V}$ \\
Medium & Tap Water \\
Zeta potential (PDMS) & $-89 \mathrm{mV}$ \\
Zeta potential (glass) & $-77 \mathrm{mV}$ \\
Conductivity of tap water & $0.05 \mathrm{~S} / \mathrm{m}$ \\
\hline
\end{tabular}

As experiments showed that $25 \mathrm{~V} / \mathrm{cm}$ produced the best results, and this value was also used in COMSOL. Since a major component of food dye is tap water, tap water rather than DI water was used as a medium. Zeta potentials of glass and PDMS were measured to be $-88 \mathrm{mV}$ to $-66 \mathrm{mV}$ and $-110 \mathrm{mV}$ to $-68 \mathrm{mV}$ respectively (Sze, Erickson et al. 2003), and the average values were used for the COMSOL model. 


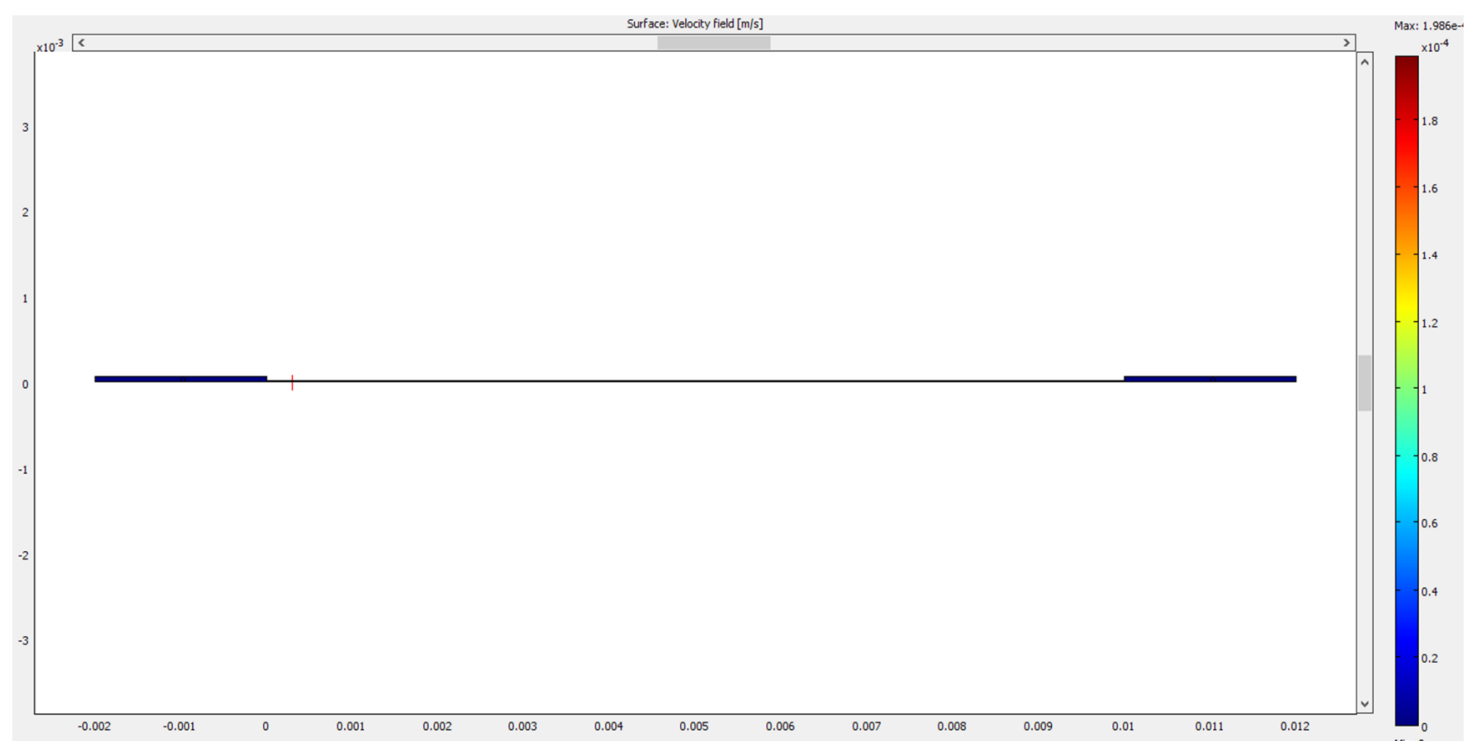

Figure 46: Cross-section of the electrode chambers and the separation chamber

A magnified section of the chip is as follows:

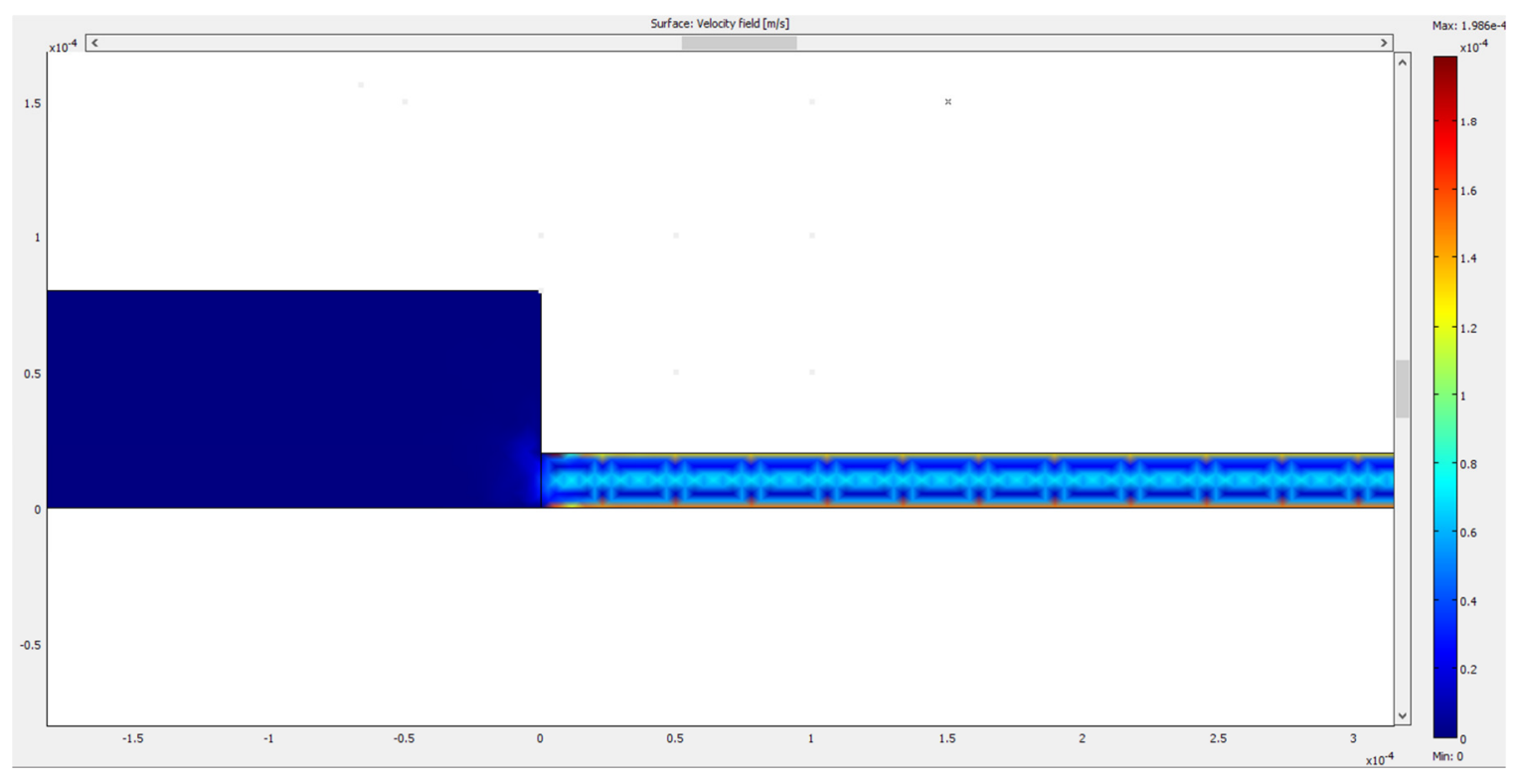

Figure 47: Magnified cross-section of the left electrode chamber and separation chamber showing the velocity profile 


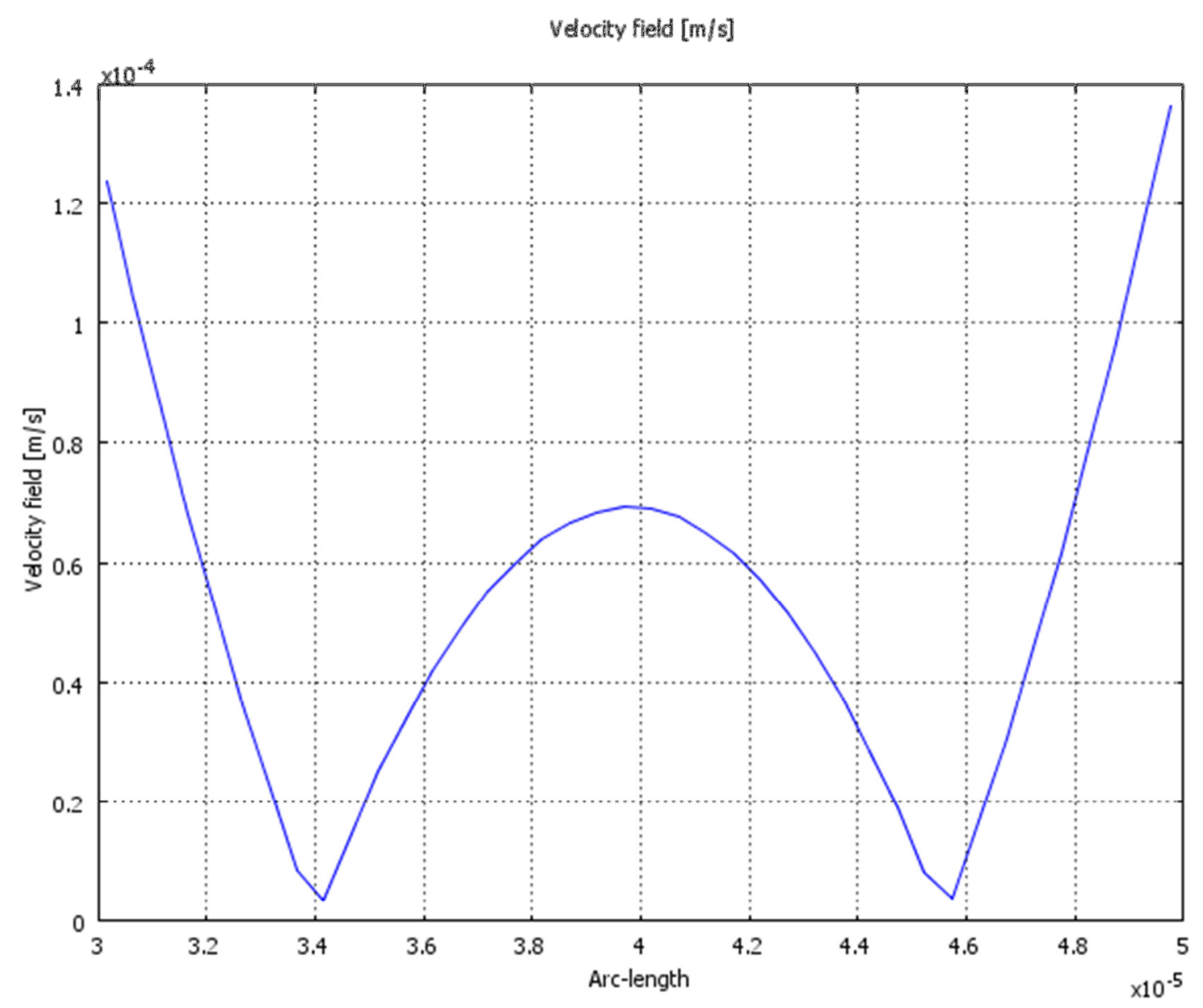

Figure 48: Vertical velocity profile of the separation chamber at $1.4 \times 10^{-4} \mathrm{~m}$

As seen in the velocity profile in Figure 48 of the separation chamber, it is possible to expect the maximum velocity at the fluid-solid interface to be $137 \mu \mathrm{m} / \mathrm{s}$. 


\section{CONCLUSION/DISCUSSION}

Initial testing was performed to analyze the behavior of the fluid in the chip without any treatment (Protocol in Section I). The chip was filled with DI water and then allowed to come to a standstill. A mixture of food dye and FluoSpheres was then injected in the separation chamber, and the FluoSpheres were allowed to settle on the bottom of the chip, which was when a voltage of $25 \mathrm{~V} / \mathrm{cm}$ was applied. After further testing, some areas of the chip were no longer filled with liquid, as seen in Figure 49.

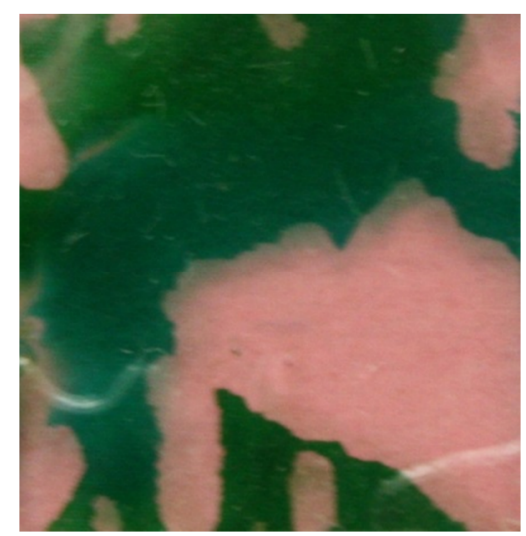

Figure 49: Chip without any treatment shortly after application of $25 \mathrm{~V} / \mathrm{cm}$.

Since the voids in the separation chamber affect the potential in the fluid, since the total volume of the fluid is much smaller than if the fluid were dispersed throughout the chip, the velocities of the FluoSpheres is impacted, and the results of this initial test were inconclusive. Following previous research procedures (Ren, Bachman et al. 2001), the chip was conditioned using $\mathrm{NaOH}$, and resulted in the chip remaining full of fluid during the experiments. By flushing the chip with $\mathrm{NaOH}$, the silanol groups on the surface of the PDMS are deprotonated and rendered hydrophilic (Cui, Horiuchi et al. 2005). This $\mathrm{NaOH}$ treatment was applied on all other experiments (see protocol in Section L). To remain consistent throughout all experiments, the mixture of food dye and FluoSpheres is used for all testing.

Several tests were then carried out to analyze the ideal voltage at which to test the chip. When a voltage of $30 \mathrm{~V} / \mathrm{cm}$ and above is applied, small particles from the food dye 
accumulate directly next to the electrode, as well as bubbles due to Joule heating as seen below in Figure 50.
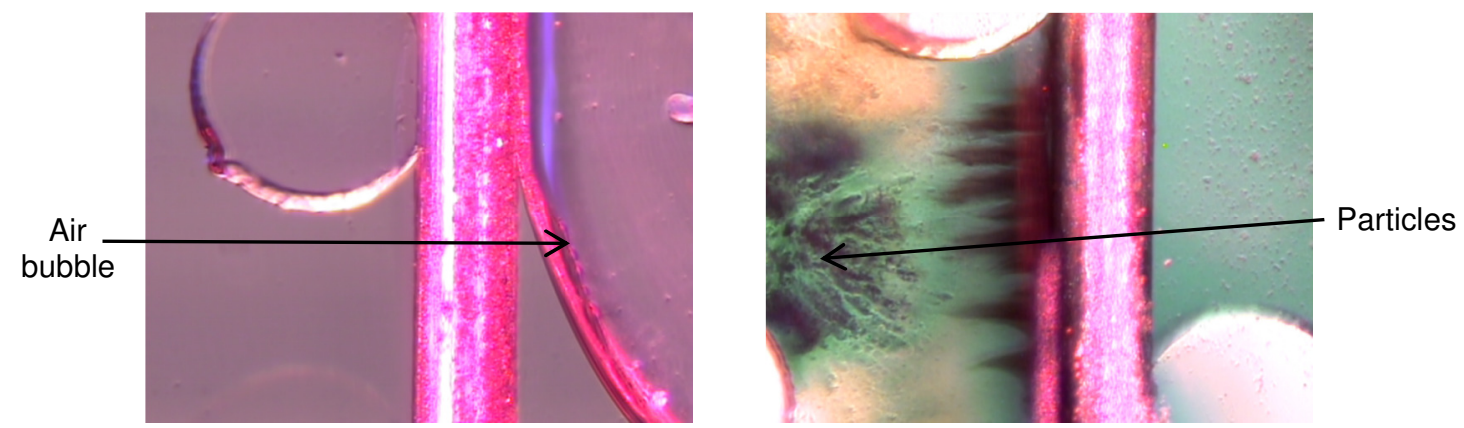

Figure 50: Formation of bubbles at the Cathode(left) and particles at the Anode (right). Experiment at $30 \mathrm{~V} / \mathrm{cm}$.

Ideally, no particles and bubbles should form in the electrode chambers. This was accomplished by performing tests at $25 \mathrm{~V} / \mathrm{cm}$ :
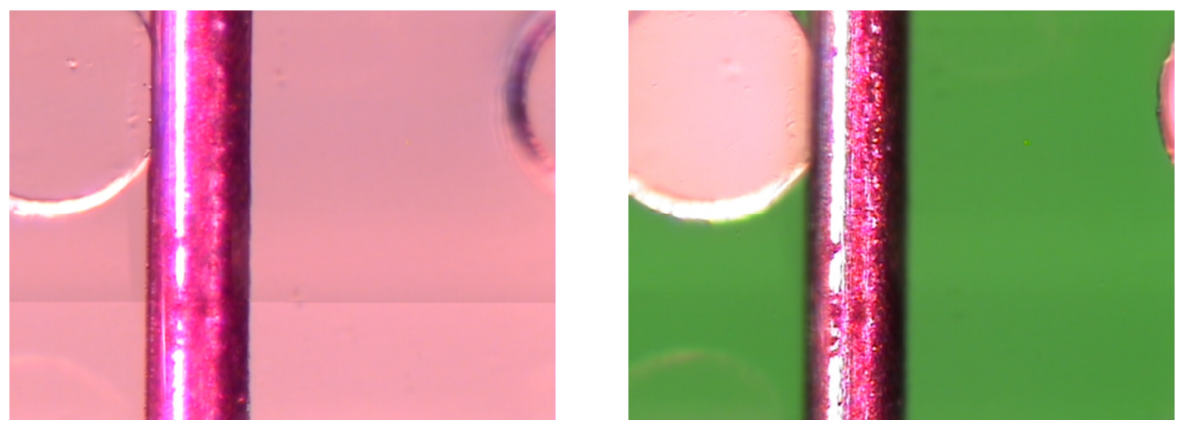

Figure 51: Lack of bubbles and particles at the electrodes (Cathode (left) and Anode (right)) when experiment is carried out at $25 \mathrm{~V} / \mathrm{cm}$.

Since particles remained in the separation chamber after the Chip Cleaning (Section $M)$, and it was necessary to reuse the chip for testing, the analysis using MC was conducted at $25 \mathrm{~V} / \mathrm{cm}$.

The flow velocity was analyzed using two methods: per Inverted Microscope and per photographs with subsequent analysis in ImageJ. In both analytical methods, the chip was initially conditioned with $\mathrm{NaOH}$ to deprotonate the chamber surfaces, and flushed with DI water. Once there was no more fluid flow, dye was inserted in the separation 
chamber. After there was no apparent fluid flow, a voltage was applied and the results were then measured.

During the treatment with $\mathrm{MC}$, the chip was initially conditioned with $\mathrm{NaOH}$, flushed with DI water and then treated with $\mathrm{MC}$. The MC was then flushed out using DI water, and the dye and FluoSpheres were injected in the separation chamber. Once the FluoSpheres settled on the bottom of the chip (glass surface), and there was no visible fluid flow, the voltage was applied and the results were recorded using the two above different methods.

Also, the chips could be used for a total of 16 times before the bond between the glass and the PDMS degraded. This caused the chambers to inflate and cause visible bulging. The chip should not be subjected to flow velocities above $1.5 \mathrm{ml} / \mathrm{min}$, since this also leads to delamination of the posts from the glass substrate.

\subsection{Via Inverted Microscope}

This method was very precise in measuring the velocity of the FluoSpheres during the application of a voltage. The inverted microscope was placed approximately in the center of the separation chamber during the experiment. In addition to the food dye, FluoSpheres were injected in the separation chamber.

The average velocities of the fluid flow in the chip without the $M C$ treatment were in the range of $913.751 \mu \mathrm{m} / \mathrm{s}-947.869 \mu \mathrm{m} / \mathrm{s}$, whereas the chip treated with $\mathrm{MC}$ showed had an average velocity of $59.634 \mu \mathrm{m} / \mathrm{s}$. Hence, the velocity of the MC-treated chip is 15.342 fold smaller than the velocity of the untreated chip.

The coefficients of variation were in the range of $0.00179 \%-0.819 \%$, indicating in a relatively narrow range of data. Due to this small coefficient of variation, the method of 
measurement is relatively precise. The treatment with $\mathrm{MC}$ clearly showed a decrease in velocity via this treatment method.

\subsection{Via Pictures and ImageJ}

The statistical comparison of the test results via ANOVA showed that mean velocities of the different treatments are the same, since $p>0.05(p=0.58)$. Therefore, the treatment of the chip with $\mathrm{MC}$ has no effect on the velocity. Despite the data following a normal distribution, the coefficients of variation were in the range of $55 \%-123 \%$, indicating that the range of data was very broad. The flow velocity for the treatment at $25 \mathrm{~V}$ was $914.9 \mu \mathrm{m} / \mathrm{s}$.

This test can easily incorporate human error, due to the method of analysis. When cropping the picture for the analysis in ImageJ, the image may be cropped incorrectly and can vary during the treatment of each picture. This would lead to an incorrect area, followed by an incorrect calculation in ImageJ. This data should be disregarded due to the high probability of human error.

\subsection{Flow Velocity comparison with COMSOL results}

Due to the high possibility of human error, the results of the ImageJ pictures will be disregarded as discussed Section 5.2. The untreated chip obtained through the Inverted Microscope method showed higher velocities than the data obtained through COMSOL modeling. COMSOL yielded a maximum $137 \mu \mathrm{m} / \mathrm{s}$, whereas the values obtained through the Inverted Microscope for no MC treatment were in the range of $913.751 \mu \mathrm{m} / \mathrm{s}$ $-947.869 \mu \mathrm{m} / \mathrm{s}$.

The COMSOL model represents an ideal case of the flow in the chip, but in the practical experiments, factors such as the solution in which the FluoSpheres were 
suspended in, and the properties of the food dye have an impact on the flow velocity. The COMSOL model was generated using tap water as the medium, since the largest ionic component contained in the separation chamber is the food dye. The main component of food dye is water, and the coefficient of conductivity of tap water was used for modeling to represent the worst-case scenario. The velocity of the inverted microscope was measured by tracking the FluoSpheres in the solution. Although the volume of the FluoSpheres is nearly negligible when compared to the overall volume of the Separation chamber, the FluoSpheres are suspended in a charged solution $(0.15 \mathrm{M}$ $\mathrm{NaOH}, 0.05 \%$ Tween, $0.02 \%$ thimerosal) which would also contributed to the bulk movement of the analyte. Although the surface charge of the FluoSpheres has not yet been documented, the FluoSpheres accelerated towards the anode during the experiments. The net flow towards the anode may be contributed to a combination of the food dye, the surface charge of the FluoSphere and the solution the FluoSpheres are suspended in.

\subsection{Final Remarks}

The design of this microfluidic chip was targeted to carry out an IEF experiment. The objectives changed throughout the project, and the chip was used for the analysis of EOF. Eliminating EOF is a very important first step in moving towards IEF, since IEF experiments cannot be successfully carried out with EOF.

The new method of "sewing" electrodes was successfully tested in this chip, and this method may be implemented in future microfluidic chips.

It was very important to conduct the experiments on a chip that may be used for the future analysis of IEF, since we established parameters at which to run the experiment, and identified potential methods of failure. This included identifying correct geometry of 
the posts in the chambers, flow velocities, concentrations of analytes, and methods of analysis.

This project showed that it was possible to reduce electroosmotic flow in a chamber by a 15-fold when treating the surface using methylcellulose.

Future recommendations: It should be considered to carry out the same experiments using markers that are free of surface charge. Since the FluoSpheres used in the experiments were submerged in Tween and $\mathrm{NaOH}$, markers used for future experiments would also need to be free of any charged solutions. Also, eliminating the need for food dye, since the major component in the dye is tap water. This would allow for a more precise measurement of the elimination of EOF via MC. For the analysis of EOF only, a simpler chamber design can be used to facilitate analysis.

The mold created for this project can be used for future projects in the Cal Poly microfluidics team, and the details of analytes and flow analysis contained in this project can be applied to future projects in clEF. 


\section{REFERENCES}

Albrecht, J. W. and K. F. Jensen (2006). "Micro free-flow IEF enhanced by active cooling and functionalized gels." Electrophoresis 27(24): 4960-4969.

Anderson, J. R., D. T. Chiu, et al. (2000). "Fabrication of topologically complex threedimensional microfluidic systems in PDMS by rapid prototyping." Anal Chem 72(14): 3158-3164.

Baldessari, F. and J. G. Santiago (2006). "Electrophoresis in nanochannels: brief review and speculation." J Nanobiotechnology 4: 12.

Bhattacharya, S., A. G. Datta, et al. (2005). "Studies on Surface Wettability of Poly(Dimethyl) Siloxane (PDMS) and Glass Under Oxygen-Plasma Treatment and Correlation With Bond Strength." J Microelectromech Syst 14(3): 590-597.

Bodas, D. and C. Khan Malek (2006). "Hydrophilization and hydrophobic recovery of PDMS by oxygen plasma and chemical treatment- An SEM investigation." Sensors and Actuators B(123): 368-373.

Breuer, K. S. (2005). Microscale diagnostic techniques. Berlin ; New York, Springer: xii, $259 \mathrm{p}$.

Cabrera, C. R., B. Finlayson, et al. (2001). "Formation of natural pH gradients in a microfluidic device under flow conditions: model and experimental validation." Anal Chem 73(3): 658-666.

Cabrera, C. R. and P. Yager (2001). "Continuous concentration of bacteria in a microfluidic flow cell using electrokinetic techniques." Electrophoresis 22(2): 355-362.

Chang, C. C., C. C. Chen, et al. (2012). "Diagnostic devices for isothermal nucleic acid amplification." Sensors (Basel) 12(6): 8319-8337.

Chin, C. D., V. Linder, et al. (2007). "Lab-on-a-chip devices for global health: past studies and future opportunities." Lab Chip 7(1): 41-57.

Cui, H., K. Horiuchi, et al. (2005). "Isoelectric focusing in a poly(dimethylsiloxane) microfluidic chip." Anal Chem 77(5): 1303-1309. 
Davey, J. and M. Lord (2003). Essential cell biology : a practical approach. Oxford ; New York, Oxford University Press.

Dertinger, S. K., D. T. Chiu, et al. (2001). "Generation of Gradients Having Complex Shapes Using Microfluidic Netowkrs." Anal Chem(73): 1240-1246.

Eddington, D. T. (2005). "Thermal aging and reduced hydrophobic recovery of polydimethylsiloxane." Sensors and Actuators B(114): 170-172.

Ewalt, K. L., R. W. Haigis, et al. (2001). "Detection of biological toxins on an active electronic microchip." Anal Biochem 289(2): 162-172.

Fadriquela, J. J. (2009). Design, Fabrication, and Implementation of a Single-Cell Capture Chamber for a Microfluidic Impedance Sensor. Master of Science in Biomedical Engineering, California Polytechnic State University.

Folch, A., B. H. Jo, et al. (2000). "Microfabricated elastomeric stencils for micropatterning cell cultures." J Biomed Mater Res 52(2): 346-353.

Fonslow, B. R., V. H. Barocas, et al. (2006). "Using channel depth to isolate and control flow in a micro free-flow electrophoresis device." Anal Chem 78(15): 53695374 .

Fonslow, B. R. and M. T. Bowser (2005). "Free-flow electrophoresis on an anodic bonded glass microchip." Anal Chem 77(17): 5706-5710.

Frost, N. W. and M. T. Bowser (2010). "Using buffer additives to improve analyte stream stability in micro free flow electrophoresis." Lab Chip 10(10): 1231-1236.

Fu, L. M., J. Y. Lin, et al. (2003). "Analysis of electroosmotic flow with step change in zeta potential." J Colloid Interface Sci 258(2): 266-275.

Hoffmann, P., H. Ji, et al. (2001). "Continuous free-flow electrophoresis separation of cytosolic proteins from the human colon carcinoma cell line LIM 1215: a non two-dimensional gel electrophoresis-based proteome analysis strategy." Proteomics 1(7): 807-818.

Hong, Y. C., H. J. Park, et al. (2010). "Plasma formation using a capillary discharge in water and its application to the sterilization of E. Coli." Physics of Plasmas 17(053502). 
Kohlheyer, D., G. A. Besselink, et al. (2006). "Free-flow zone electrophoresis and isoelectric focusing using a microfabricated glass device with ion permeable membranes." Lab Chip 6(3): 374-380.

Krivankova, L. and P. Bocek (1998). "Continuous free-flow electrophoresis." Electrophoresis 19(7): 1064-1074.

Kushimoto, T., V. Basrur, et al. (2001). "A model for melanosome biogenesis based on the purification and analysis of early melanosomes." Proc Natl Acad Sci U S A 98(19): 10698-10703.

Laberge, M. (1998). "Intrinsic protein electric fields: basic non-covalent interactions and relationship to protein-induced Stark effects." Biochim Biophys Acta 1386(2): 305-330.

Li, Y., D. L. DeVoe, et al. (2003). "Dynamic analyte introduction and focusing in plastic microfluidic devices for proteomic analysis." Electrophoresis 24(1-2): 193199.

Liu, C., X. Xu, et al. (2008). "Multilayer hydroxyethyl cellulose-adsorbed coating on poly(dimethylsiloxane) microchips for protein separation." Can J Anal Sci Spect 53(4): 171-178.

Liu, X., Z. Sosic, et al. (1996). "Capillary isoelectric focusing as a tool in the examination of antibodies, peptides and proteins of pharmaceutical interest." $\underline{\mathrm{J}}$ Chromatogr A 735(1-2): 165-190.

Liu, Y., C. D. Garcia, et al. (2003). "Recent progress in the development of muTAS for clinical analysis." Analyst 128(8): 1002-1008.

Macounova, K., C. R. Cabrera, et al. (2000). "Generation of natural pH gradients in microfluidic channels for use in isoelectric focusing." Anal Chem 72(16): 3745-3751.

Maheswaran, S., L. V. Sequist, et al. (2008). "Detection of mutations in EGFR in circulating lung-cancer cells." N Engl J Med 359(4): 366-377.

McDonald, J. C. and G. M. Whitesides (2002). "Poly(dimethylsiloxane) as a material for fabricating microfluidic devices." Acc Chem Res 35(7): 491-499. 
Momen, G. and M. Farzaneh (2010). "Survey of Micro/Nano Filler Use to Improve Silicone Rubber for Outdoor Insulators." Rev Adv Mater Sci 38: 1-13.

Probstein, R. F. (2003). Physicochemical hydrodynamics : an introduction. Hoboken, N.J., Wiley-Interscience.

Raymond, D. E., A. Manz, et al. (1996). "Continuous separation of high molecular weight compounds using a microliter volume free-flow electrophoresis microstructure." Anal Chem 68(15): 2515-2522.

Ren, Z. L., M. A. Bachman, et al. (2001). "Electroosmotic properties of microfluidic channels composed of poly(dimethylsiloxane)." J Chromatogr A(726): 117125.

Shen, K., X. Chen, et al. (2005). "A microchip-based PCR device using flexible printed circuit technology." Sensors and Actuators(105): 251-258.

Sze, A., D. Erickson, et al. (2003). "Zeta-potential measurement using the Smoluchowski equation and the slope of the current-time relationship in electroosmotic flow." J Colloid Interface Sci 261(2): 402-410.

Tang, G., D. Yan, et al. (2006). "Assessment of Joule heating and its effects on electroosmotic flow and electrophoretic transport of solutes in microfluidic channels." Electrophoresis 27(3): 628-639.

Vickers, J. A., M. M. Caulum, et al. (2006). "Generation of hydrophilic poly(dimethylsiloxane) for high-performance microchip electrophoresis." Anal Chem 78(21): 7446-7452.

Walker, G. M. and D. J. Beebe (2002). "An evaporation-based microfluidic sample concentration method." Lab Chip 2(2): 57-61.

Weber, K. and M. Osborn (1969). "The reliability of molecular weight determinations by dodecyl sulfate-polyacrylamide gel electrophoresis." J Biol Chem 244(16): 4406-4412.

Xu, Y., C. X. Zhang, et al. (2003). "Sub-second isoelectric focusing in free flow using a microfluidic device." Lab Chip 3(4): 224-227.

Zhang, C. X. and A. Manz (2003). "High-speed free-flow electrophoresis on chip." Anal Chem 75(21): 5759-5766. 


\section{APPENDICES}

A. Reagents

- Bovine Hemoglobin

- 5 g Bovine Hemoglobin, Prod \# 16891

- Purchased from: USBWEB, Cleveland, Ohio

- $\underline{\mathrm{NaOH}}$

- 500 g Sodium Hydroxide, Cat. No. NC-0874

- Purchased from: Science Company, Denver, CO

- Methylcellulose

- $100 \mathrm{~g}$ Methylcellulose, $400 \mathrm{cP}$ at 2\%, Cat. No. M0262-100G

- Purchased from: Sigma-Aldrich, St. Louis, MO

- Bovine Serum Albumin

- 5 mg BSA/Bodipy Conjugate, Cat. No. A2750

- Purchased from: Invitrogen, Carlsbad, CA

- $\quad$ Anode Buffer

- 250 ml 10 x IEF Anode Buffer, \#161-761

- Purchased from: Bio-Rad, Hercules, CA

- Ampholyte

- 10 ml Bio-Lyte 3/10 Ampholyte \#163-1112

- Purchased from: Bio-Rad, Hercules, CA

- $\quad$ FluoSpheres

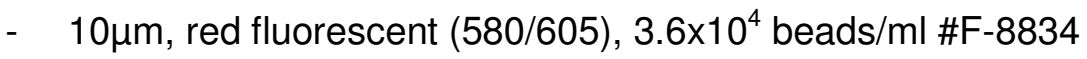

- Purchased from: Invitrogen, Grand Island, NY 


\section{B. Disposable Materials}

- Tygon Tubing

- Dispensing Needles:

- $\quad$ Type 304 SS, 23 Gauge, \#75165A684

- $10 \mathrm{ml}$ syringes

- $3 \mathrm{ml}$ syringes

- 40 Gauge Copper 99.99\% CDA 101

- California Fine Wire Company (Grover Beach, CA), \#100156

\section{Equipment}

- Inverted Microscope

- Voltage Sequencer

- Hot Plate

- Computer

\section{Facilities at Cal Poly}

- For testing and some assembly:

- St. Jude Medical Lab in the Advanced Technology Labs

- For PDMS pouring and some assembly:

- Materials Engineering clean room (clean level: 1000) (Bldg. 41)

\section{E. Protocol: Pouring PDMS}

This protocol was carried out in the Material Engineering Clean room at Cal Poly using guidelines set by Joshua Jed-Fadriquela's protocol (Fadriquela 2009):

1) Calculate total volume needed to pour a $0.5 \mathrm{~cm}$ thick chip: 
2) The volume, V, of PDMS needed is computed by measuring the diameter of the wafer and applying the equation $\mathrm{V}=\pi \mathrm{r}^{2} \mathrm{~h}$, where $\mathrm{r}$ is the radius, and $\mathrm{h}$ the height of the PDMS chip.

3) Applying this to the $10.16 \mathrm{~cm}$ (4 inch) diameter chip, the volume of PDMS needed was $40.54 \mathrm{~cm} 3$, a total of $40.54 \mathrm{ml}$.

4) The ratio of PDMS base:hardener is $10: 1$, which yields amounts of $37 \mathrm{ml}$ base to 3.7 $\mathrm{ml}$ hardener.

F. Protocol: $100 \mathrm{ml} \mathbf{1 M}$ Sodium Hydroxide $(\mathrm{NaOH})$

Materials needed:

$4.08 \mathrm{~g} \mathrm{NaOH}$

$95.92 \mathrm{ml}$ Dl water

$100 \mathrm{ml}$ beaker
Stir bar

Stir plate

Scale

$100 \mathrm{ml}$ Calibrated beaker

1) Measure $\mathrm{NaOH}$

2) Measure DI water

3) Place water in beaker with stirbar over stirplate

4) Add $\mathrm{NaOH}$ to the water while stirring with the stirplate

5) It is done when the solid $\mathrm{NaOH}$ pieces are completely dissolved

G. Protocol: $100 \mathrm{ml} 1 \%$ Methylcellulose (MC)

\begin{tabular}{ll}
\hline Materials needed & \\
\hline Thermometer & Stirbar \\
Hotplate & $100 \mathrm{ml}$ Calibrated beaker \\
\hline
\end{tabular}

1) Measure $98 \mathrm{ml}$ water

2) Measure $1 \mathrm{mg} \mathrm{MC}$

3) Heat about $30 \mathrm{ml}$ water to at least $80^{\circ} \mathrm{C}$

4) Add $2 \mathrm{mg} \mathrm{MC}$ powder to hot water with agitation

5) Agitate mixture until particles are thoroughly wetted and evenly dispersed 
6) For complete solubilization, the remainder of the water is added as cold water or ice to lower the temperature of the dispersion. Once the dispersion reaches the temperature at which that particular MC product becomes water soluble, the powder begins to hydrate and the viscosity increases. The solution should be cooled to 0-5 oC for 20-40 min

7) Continue agitation for at least 30 min after the proper temperature is reached.

8) Allow solution to stand overnight for complete dispersion

\section{H. Protocol: Food dye only}

1) Prepare a 1:3 (McCormick's green food dye-DI water) mixture by combining:
a) $3.33 \mathrm{ml}$ food dye
b) $6.66 \mathrm{ml} \mathrm{DI}$ water

\section{Protocol: Food dye with FluoSpheres}

1) Prepare a 1:3 (McCormick's green food dye-DI water) mixture by combining:
a) $0.33 \mathrm{ml}$ food dye
b) $0.66 \mathrm{ml} \mathrm{DI}$ water
c) $0.01 \mathrm{cc} 10 \mu \mathrm{m}$ fluorospheres

J. Protocol for Test 1: Preliminary Testing to analyze EOF without treatment at $25 \mathrm{~V} / \mathrm{cm}$

\section{Per Inverted Microscope}
Prepare the following sets of syringes:
Syringe A: 1EA $1 \mathrm{ml}$ syringe with food dye and FluoSpheres (Section I)
Syringe B: 2EA $10 \mathrm{ml}$ syringes with DI water

\section{Program the Syringe Pumps located at the following chambers with the respective parameters: \\ Electrode chambers: Volume: $10 \mathrm{ml}$; Rate: $1.2 \mathrm{ml} / \mathrm{min}$ \\ Separation chamber: Volume: $10 \mathrm{ml}$; Rate: $0.5 \mathrm{ml} / \mathrm{min}$}


Imaging:

Position the inverted microscope approximately at the center of the Separation chamber, and record the data at 15 second intervals

1) Briefly rinse the chip by connecting each of the Syringes B to each electrode inlet, and running the syringe pumps for approximately 5 minutes.

2) Remove syringes $B$ from the electrode inlets

3) Let the fluid in the chip come to a standstill

4) Connect syringe A to Separation chamber and slowly inject approximately $0.01 \mathrm{cc}$

5) Let the fluid in the chip come to a standstill

6) Turn on the Voltmeter to $25 \mathrm{~V} / \mathrm{cm}$

7) Ensure good visualization of FluoSpheres. If necessary, move microscope to an area with more FluoSpheres

8) Record the velocity of the FluoSpheres

K. Protocol for Test 2: Analysis with $\mathrm{NaOH}$ treatment at $25 \mathrm{~V} / \mathrm{cm}, 50 \mathrm{~V} / \mathrm{cm}$, and $75 \mathrm{~V} / \mathrm{cm}$

To analyze the behavior of the fluid in the chip at different voltages without treatment, the following protocols were carried out at $25 \mathrm{~V} / \mathrm{cm}, 50 \mathrm{~V} / \mathrm{cm}$, and $75 \mathrm{~V} / \mathrm{cm}$.

Reference Section A for the preparation of reagents.

Per Inverted Microscope $\quad$ Per Pictures and ImageJ 
Prepare the following sets of syringes:

Syringe A: 2EA 10ml Food Dye

Syringe B: $2 \mathrm{EA} 10 \mathrm{ml} \mathrm{1 \%} \mathrm{MC}$

Syringe C: $2 \mathrm{EA} 10 \mathrm{ml} 1 \mathrm{M} \mathrm{NaOH}$

Syringe D: 2EA $10 \mathrm{ml}$ DI water

Syringe E: 1EA 1cc syringe with $0.03 \mathrm{cc}$ of $10 \mu \mathrm{m}$ fluorospheres

Program the Syringe Pumps located at the following chambers with the respective parameters:

Electrode chambers: Volume: $10 \mathrm{ml}$; Rate: $1.2 \mathrm{ml} / \mathrm{min}$

Separation chamber: Volume: $10 \mathrm{ml}$; Rate: $0.5 \mathrm{ml} / \mathrm{min}$

Imaging:

Position the inverted microscope approximately at the center of the Separation chamber, and record the data at 15 second intervals

1) Connect each of the syringes $C$ to each electrode inlet and run the syringes until the entire chip is filled with $\mathrm{NaOH}$ for 10 min.

2) Remove syringes $C$ from electrode inlets

3) Connect syringes $D$ to electrode inlets

4) Flush $\mathrm{NaOH}$ out with $\mathrm{DI}$ water Syringes $D$ for 5 min

5) Disconnect Syringes D from the
Prepare the following sets of syringes:

Syringe A: 2EA 10ml Food Dye

Syringe B: $2 \mathrm{EA} 10 \mathrm{ml} \mathrm{1 \%} \mathrm{MC}$

Syringe C: $2 \mathrm{EA} 10 \mathrm{ml} 1 \mathrm{M} \mathrm{NaOH}$

Syringe D: 2EA $10 \mathrm{ml} \mathrm{DI}$ water

Syringe E: 1EA 1cc syringe with $0.03 \mathrm{cc}$ of $10 \mu \mathrm{m}$ fluorospheres
Program the Syringe Pumps located at the chambers with the following parameters:

Electrode chambers: Volume: $10 \mathrm{ml}$; Rate: $1.2 \mathrm{ml} / \mathrm{min}$

Separation chamber: Volume: $10 \mathrm{ml}$; Rate: $0.5 \mathrm{ml} / \mathrm{min}$
Imaging:

Place a pink sheet of paper below the chip to increase the contrast of the dye. Position a camera on a tripod above the chip.

1) Connect each of the syringes $C$ to each electrode inlet and run the syringes until the entire chip is filled with $\mathrm{NaOH}$ for 10 min.

2) Remove syringes $C$ from electrode inlets

3) Connect syringes $D$ to electrode inlets

4) Flush $\mathrm{NaOH}$ out with $\mathrm{DI}$ water Syringes $D$ for 5 min

5) Disconnect Syringes $D$ from the 
electrode inlets

6) Connect Syringes $A$ to the electrode inlets and saturate chip with green dye

7) Stop syringe pump and disconnect syringes to allow all fluid in the chip to come to a standstill

8) Connect Syringe E to the Separation chamber, and gently inject approximately $0.01 \mathrm{cc}$ fluorospheres

9) Ensure good visualization of fluorospheres. If necessary, move microscope to an area with more fluorospheres

10) Allow for all fluid to come to a standstill

11) Turn on volt meter to $25 \mathrm{~V} / \mathrm{cm} /$ $50 \mathrm{~V} / \mathrm{cm} / 75 \mathrm{~V} / \mathrm{cm}$

12) Record velocities of fluorospheres electrode inlets

6) Connect Syringes $A$ to the electrode inlets and saturate chip with green dye

7) Stop syringe pump and disconnect syringes to allow all fluid in the chip to come to a standstill

8) Turn on volt meter to $25 \mathrm{~V} / \mathrm{cm}$ / $50 \mathrm{~V} / \mathrm{cm} / 75 \mathrm{~V} / \mathrm{cm}$

9) Take a picture of the chip

L. Protocol for Test 3: Analysis with $\mathrm{NaOH}$ and MC surface treatments at 25 $\mathrm{V} / \mathrm{cm}$

To analyze the behavior of fluid in the chip with the MC treatment, the experiment was conduted at $25 \mathrm{~V} / \mathrm{cm}$.

Reference Section A for the preparation of reagents. 


\section{Per Inverted Microscope}

\section{Prepare the following sets of} syringes:

Syringe A: 2EA 10ml Food Dye

Syringe B: $2 E A 10 \mathrm{ml} \mathrm{1 \%} \mathrm{MC}$

Syringe C: $2 \mathrm{EA} 10 \mathrm{ml} 1 \mathrm{M} \mathrm{NaOH}$

Syringe D: 2EA 10ml DI water

Syringe E: 1EA 1cc syringe with $0.03 \mathrm{cc}$ of $10 \mu \mathrm{m}$ fluorospheres

Program the Syringe Pumps located at the following chambers with the respective parameters:

Electrode chambers: Volume: $10 \mathrm{ml}$; Rate: $1.2 \mathrm{ml} / \mathrm{min}$

Separation chamber: Volume: $10 \mathrm{ml}$; Rate: $0.5 \mathrm{ml} / \mathrm{min}$

Imaging:

Position the inverted microscope approximately at the center of the Separation chamber, and record the data at 15 second intervals

1) Connect each of the syringes $C$ to each electrode inlet and run the syringes until the entire chip is filled with $\mathrm{NaOH}$ for 10 min.

2) Remove syringes $C$ from electrode inlets

3) Connect syringes $D$ to electrode inlets

4) Flush $\mathrm{NaOH}$ out with $\mathrm{DI}$ water Syringes $D$ for 5 min

5) Disconnect Syringes $D$ from the

\section{Per Pictures and ImageJ}

Prepare the following sets of syringes:

Syringe A: 2EA 10ml Food Dye

Syringe B: 2EA $10 \mathrm{ml} 1 \% \mathrm{MC}$

Syringe C: $2 \mathrm{EA} 10 \mathrm{ml} 1 \mathrm{M} \mathrm{NaOH}$

Syringe D: 2EA $10 \mathrm{ml} \mathrm{DI}$ water

Syringe E: 1EA 1cc syringe with $0.03 \mathrm{cc}$ of $10 \mu \mathrm{m}$ fluorospheres

Program the Syringe Pumps located at the chambers with the following parameters:

Electrode chambers: Volume: $10 \mathrm{ml}$; Rate: $1.2 \mathrm{ml} / \mathrm{min}$

Separation chamber: Volume: $10 \mathrm{ml}$; Rate: $0.5 \mathrm{ml} / \mathrm{min}$

\section{Imaging:}

Place a pink sheet of paper below the chip to increase the contrast of the dye. Position a camera on a tripod above the chip.

1) Connect each of the syringes $C$ to each electrode inlet and run the syringes until the entire chip is filled with $\mathrm{NaOH}$ for 10 min.

2) Remove syringes $C$ from electrode inlets

3) Connect syringes $D$ to electrode inlets

4) Flush $\mathrm{NaOH}$ out with $\mathrm{DI}$ water Syringes $D$ for 5 min

5) Disconnect Syringes $D$ from the Page 94 
electrode inlets

6) Connect Syringes $A$ to the electrode inlets and saturate chip with green dye

7) Stop syringe pump and disconnect syringes to allow all fluid in the chip to come to a standstill

8) Connect Syringe $E$ to the Separation chamber, and gently inject approximately $0.01 \mathrm{cc}$ fluorospheres

9) Ensure good visualization of fluorospheres. If necessary, move microscope to an area with more fluorospheres

10)Allow for all fluid to come to a standstill

11)Turn on volt meter to $25 \mathrm{~V} / \mathrm{cm} /$ $50 \mathrm{~V} / \mathrm{cm} / 75 \mathrm{~V} / \mathrm{cm}$

12)Record velocities of fluorospheres

\section{Protocol: Chip Cleaning}

After running the chip with food dye, small pieces of food dye often adhere to the walls of the chip. The following protocol was used to clean the chip of particles after each experimental run:

Prepare the following sets of syringes:

Syringe A: $3 E A 10 \mathrm{ml}$ syringe with DI water

Syringe $\mathrm{B}: \quad 3 \mathrm{EA} 10 \mathrm{ml}$ syringes with $1 \mathrm{M} \mathrm{NaOH}$ (Section F)

Syringe C: 3EA empty electrode inlets

6) Connect Syringes $A$ to the electrode inlets and saturate chip with green dye

7) Stop syringe pump and disconnect syringes to allow all fluid in the chip to come to a standstill

8) Turn on volt meter to $25 \mathrm{~V} / \mathrm{cm} /$ $50 \mathrm{~V} / \mathrm{cm} / 75 \mathrm{~V} / \mathrm{cm}$

9) Take a picture of the chip 
Program the Syringe Pumps located at the following chambers with the respective parameters:

Electrode chambers: Volume: $10 \mathrm{ml}$; Rate: $0.1 \mathrm{ml} / \mathrm{min}$

Separation chamber: Volume: $10 \mathrm{ml}$; Rate: $0.1 \mathrm{ml} / \mathrm{min}$

1) Connect syringes $A$ to the separation and the electrode chamber inlets

2) Run the syringe pumps with syringes $A$ until the chambers are visually clear of particles

3) Disconnect syringes $A$ from the chip

4) Connect and run syringes $B$ for 5 min

5) Stop the syringe pumps, and allow the $\mathrm{NaOH}$ to remain in the chambers for 5 min

6) Run syringes $B$ again for 5 min

7) Disconnect syringes $B$ and connect syringes $A$

8) Run syringes $\mathrm{A}$ for $30 \mathrm{~min}$

9) Connect syringes $\mathrm{C}$ and to push out the DI water and to fill the chambers with air

10) Run empty syringes $A$ until all liquid is pushed out of the chip 
N. Test Results: Test data with $\mathrm{NaOH}$ treatment at $25 \mathrm{~V} / \mathrm{cm}$

Inverted Microscope: $\mathrm{NaOH}$ treatment at $25 \mathrm{~V} / \mathrm{cm}$

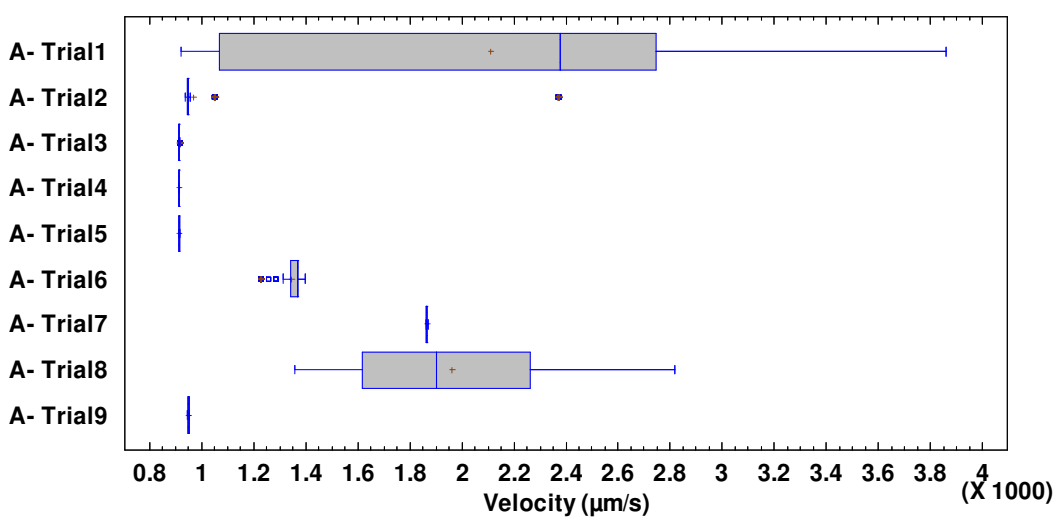

Figure 52: Box-and-Whisker plot of Inverted Microscope trials of $\mathrm{NaOH}$ treatment at $25 \mathrm{~V} / \mathrm{cm}$ 


\begin{tabular}{|c|c|c|c|c|c|c|}
\hline \multicolumn{7}{|l|}{ Summary Statistics } \\
\hline & A-Triall & A- Trial2 & A- Trial3 & A- Trial4 & A- Trial5 & A-Trial6 \\
\hline Count & 868 & 844 & 591 & 392 & 46 & 360 \\
\hline \begin{tabular}{|l} 
Average \\
\end{tabular} & 2110.42 & 970.559 & 914.638 & 912.82 & 914.906 & 1345.67 \\
\hline Standard deviation & 881.63 & 169.276 & 0.782876 & 0.0903393 & 0.0861921 & 56.2108 \\
\hline Coeff. of variation & $41.7751 \%$ & $17.4411 \%$ & $0.0855941 \%$ & $0.00989673 \%$ & $0.00942086 \%$ & $4.17716 \%$ \\
\hline Minimum & 921.225 & 938.585 & 913.699 & 912.68 & 914.726 & 1227.47 \\
\hline Maximum & 3857.34 & 2373.21 & 916.818 & 913.024 & 915.017 & 1398.85 \\
\hline Range & 2936.12 & 1434.63 & 3.11939 & 0.344253 & 0.290937 & \begin{tabular}{|l|}
171.378 \\
\end{tabular} \\
\hline Stnd. skewness & 0.597057 & 95.8967 & 15.6413 & 3.67512 & -1.87183 & -9.27116 \\
\hline \multirow[t]{2}{*}{ Stnd. kurtosis } & -6.32482 & 380.799 & 9.474 & -4.9027 & -0.980469 & 0.479401 \\
\hline & A- Trial7 & A-Trials & A-Trial9 & & & \\
\hline Count & 1427 & 864 & 516 & & & \\
\hline Average & 1865.13 & 1962.08 & 950.195 & & & \\
\hline \begin{tabular}{|l|} 
Standard deviation \\
\end{tabular} & 2.5793 & 399.73 & 1.73995 & & & \\
\hline Coeff. of variation & $0.138291 \%$ & $20.3728 \%$ & $0.183116 \%$ & & & \\
\hline Minimum & 1859.67 & 1356.15 & 946.795 & & & \\
\hline Maximum & 1871.21 & 2817.25 & 953.134 & & & \\
\hline Range & \begin{tabular}{|l|}
11.5459 \\
\end{tabular} & 1461.09 & 6.33953 & & & \\
\hline Stnd. skewness & 3.30506 & 4.66076 & -2.32998 & & & \\
\hline Stnd. kurtosis & -6.18439 & -5.5719 & -4.08532 & & & \\
\hline
\end{tabular}

\section{The StatAdvisor}

This table shows summary statistics for each of the selected data variables. It includes measures of central tendency, measures of variability, and measures of shape. Of particular interest here are the standardized skewness and standardized kurtosis, which can be used to determine whether the sample comes from a normal distribution. Values of these statistics outside the range of -2 to +2 indicate significant departures from normality, which would tend to invalidate many of the statistical procedures normally applied to this data. In this case, the following variables show standardized skewness values outside the expected range:
A- Trial2
A- Trial3
A- Trial4
A- Trial6
A- Trial7
A- Trial8
A- Trial9

The following variables show standardized kurtosis values outside the expected range:
A- Trial1
A- Trial2
A- Trial3
A- Trial4
A- Trial7
A- Trial8
A- Trial9

To make the variables more normal, you might try a transformation such as LOG(Y), SQRT(Y), or 1/Y.

Figure 53: Data summary of all test runs with $\mathrm{NaOH}$ treatment at $25 \mathrm{~V} / \mathrm{cm}$ 
O. Test Results: Test data with $\mathrm{NaOH}$ treatment at $50 \mathrm{~V} / \mathrm{cm}$

Inverted Microscope: $\mathrm{NaOH}$ treatment at $50 \mathrm{~V} / \mathrm{cm}$

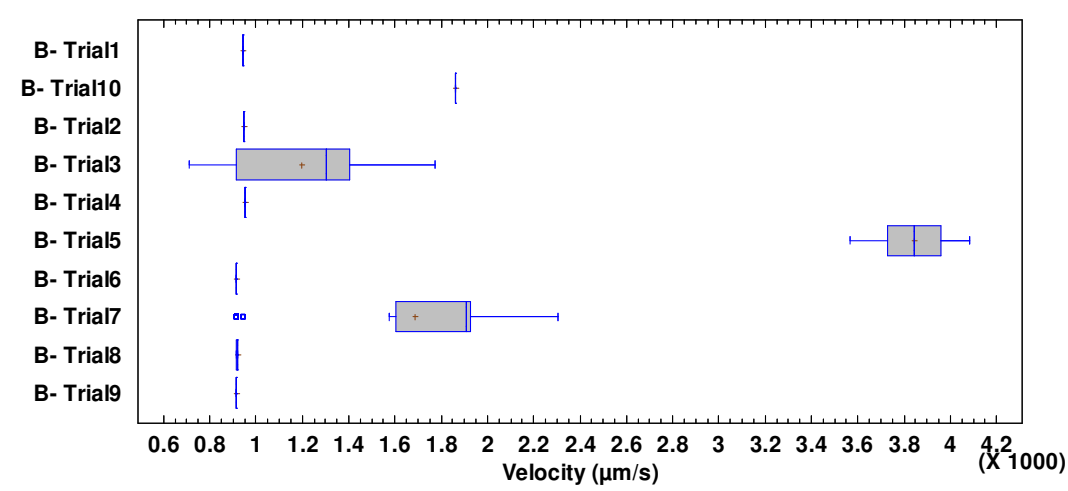

Figure 54: Box-and-Whisker plot of Inverted Microscope trials of $\mathrm{NaOH}$ treatment at $50 \mathrm{~V} / \mathrm{cm}$ 


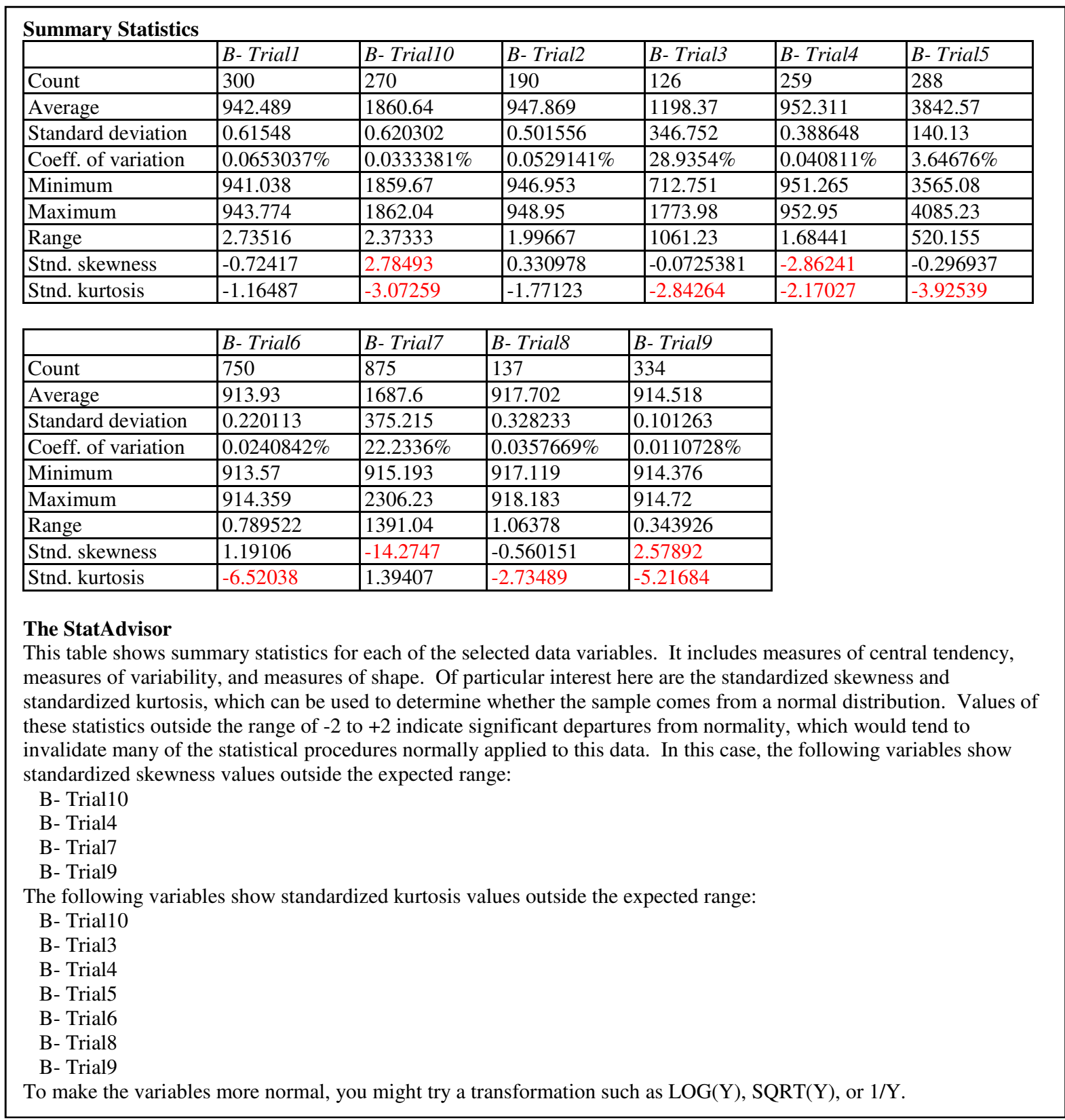

Figure 55: Data summary of all test runs with $\mathrm{NaOH}$ treatment at $50 \mathrm{~V} / \mathrm{cm}$ 
P. Test Results: Test data with $\mathrm{NaOH}$ treatment at $75 \mathrm{~V} / \mathrm{cm}$

Inverted Microscope: $\mathrm{NaOH}$ treatment at $75 \mathrm{~V} / \mathrm{cm}$

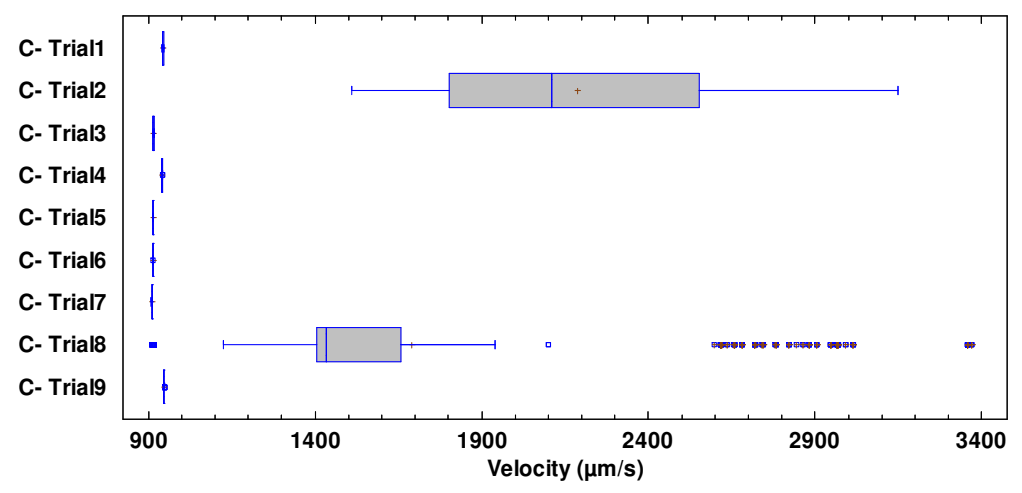

Figure 56: Box-and-Whisker plot of Inverted Microscope trials of $\mathrm{NaOH}$ treatment at $75 \mathrm{~V} / \mathrm{cm}$ 


\begin{tabular}{|c|c|c|c|c|c|c|}
\hline \multicolumn{7}{|l|}{ Summary Statistics } \\
\hline & C-Triall & C-Trial2 & C-Trial3 & C-Trial4 & C-Trial5 & $C$ - Trial6 \\
\hline Count & 250 & 675 & 318 & 182 & 554 & 222 \\
\hline Average & 943.12 & 2187.99 & 915.121 & 940.713 & 913.683 & 913.751 \\
\hline Standard deviation & 1.70043 & 461.646 & 0.803035 & 0.689301 & 0.0502327 & 0.0163995 \\
\hline Coeff. of variation & $0.180299 \%$ & $21.0991 \%$ & $0.0877519 \%$ & $0.0732743 \%$ & $0.00549783 \%$ & $0.00179475 \%$ \\
\hline Minimum & 939.23 & 1510.6 & 913.862 & 939.475 & 913.574 & 913.702 \\
\hline Maximum & 946.88 & 3148.14 & 916.634 & 942.921 & 913.783 & 913.787 \\
\hline Range & 7.65077 & 1637.54 & 2.7715 & 3.44535 & 0.208804 & 0.0849389 \\
\hline Stnd. skewness & 1.57893 & 4.07037 & 2.12231 & 3.72205 & -2.29677 & -0.702906 \\
\hline \multirow[t]{2}{*}{\begin{tabular}{|l} 
Stnd. kurtosis \\
\end{tabular}} & -1.94439 & -5.13837 & -3.70785 & 1.09245 & -4.79831 & -1.37707 \\
\hline & C- Trial7 & $C$-Trial8 & C-Trial9 & & & \\
\hline \begin{tabular}{|l|} 
Count \\
\end{tabular} & 260 & 722 & 272 & & & \\
\hline \begin{tabular}{|l|} 
Average \\
\end{tabular} & 909.669 & 1690.03 & 945.761 & & & \\
\hline Standard deviation & 0.640448 & 625.921 & 0.710382 & & & \\
\hline Coeff. of variation & $0.0704045 \%$ & $37.0361 \%$ & $0.0751122 \%$ & & & \\
\hline Minimum & 908.637 & 909.417 & 944.676 & & & \\
\hline Maximum & 910.577 & 3369.58 & 948.394 & & & \\
\hline Range & 1.93995 & 2460.16 & 3.71733 & & & \\
\hline Stnd. skewness & -2.29399 & 13.158 & 6.72228 & & & \\
\hline Stnd. kurtosis & -4.22221 & 2.80381 & 4.62926 & & & \\
\hline \multicolumn{7}{|l|}{ The StatAdvisor } \\
\hline $\begin{array}{l}\text { This table shows sur } \\
\text { measures of variabil } \\
\text { standardized kurtosi } \\
\text { these statistics outsi } \\
\text { invalidate many of th } \\
\text { standardized skewne } \\
\text { C- Trial2 } \\
\text { C- Trial3 } \\
\text { C- Trial4 } \\
\text { C- Trial5 } \\
\text { C- Trial7 } \\
\text { C- Trial8 } \\
\text { C- Trial9 }\end{array}$ & $\begin{array}{l}\text { nary statistics } \\
\text {, and measure } \\
\text { which can be } \\
\text { the range of - } \\
\text { statistical pro } \\
\text { values outsid }\end{array}$ & $\begin{array}{l}\text { or each of the } \\
\text { of shape. Of } \\
\text { sed to determi } \\
\text { to }+2 \text { indicate } \\
\text { edures normal } \\
\text { the expected }\end{array}$ & $\begin{array}{l}\text { elected data var } \\
\text { orticular intere } \\
\text { e whether the s } \\
\text { significant depa } \\
\text { y applied to this } \\
\text { ange: }\end{array}$ & $\begin{array}{l}\text { ables. It incluc } \\
\text { here are the st } \\
\text { mple comes fro } \\
\text { tures from nor } \\
\text { data. In this ca }\end{array}$ & $\begin{array}{l}\text { measures of ce } \\
\text { dardized skewn } \\
\text { a normal distril } \\
\text { lity, which wou } \\
\text { the following }\end{array}$ & $\begin{array}{l}\text { ral tendency, } \\
\text { s and } \\
\text { tion. Values of } \\
\text { tend to } \\
\text { riables show }\end{array}$ \\
\hline \multicolumn{7}{|c|}{ The following variables show standardized kurtosis values outside the expected range: } \\
\hline \multicolumn{7}{|l|}{ C- Trial2 } \\
\hline \multicolumn{7}{|l|}{ C- Trial3 } \\
\hline \multicolumn{7}{|l|}{ C- Trial5 } \\
\hline \multicolumn{7}{|l|}{ C- Trial7 } \\
\hline \multicolumn{7}{|l|}{ C- Trial8 } \\
\hline \multicolumn{7}{|l|}{ C- Trial9 } \\
\hline To make the variable & ore normal, & might try & nsformati & $\mathrm{h}$ as $\mathrm{LOG}$ & $\mathrm{RT}(\mathrm{Y}$ & \\
\hline
\end{tabular}

Figure 57: Data summary of all test runs with $\mathrm{NaOH}$ treatment at $75 \mathrm{~V} / \mathrm{cm}$ 


\section{Q. Test Results: Test data with $\mathrm{NaOH}$ and $\mathrm{MC}$ treatment at $25 \mathrm{~V} / \mathrm{cm}$}

Inverted Microscope: $\mathrm{NaOH}$ and $\mathrm{MC}$ treatment at $25 \mathrm{~V} / \mathrm{cm}$

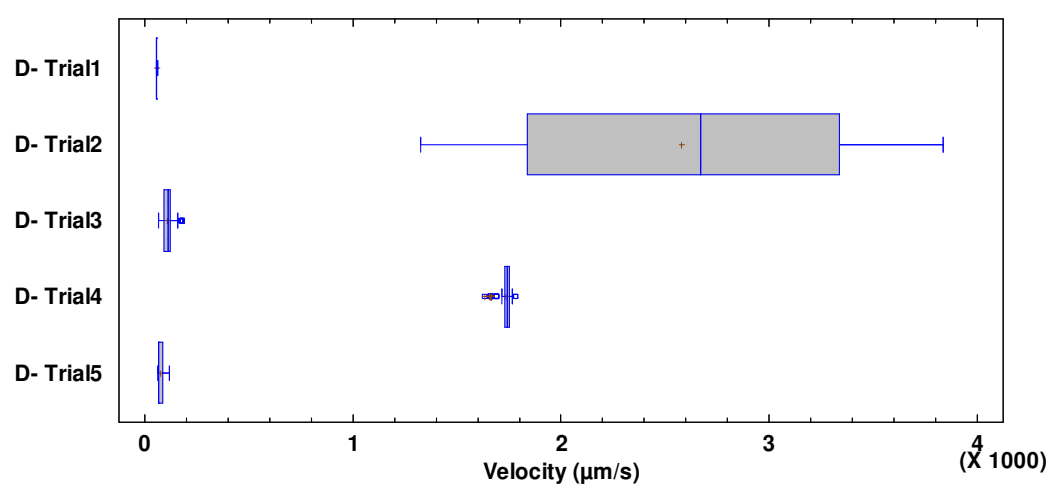

Figure 58: Box-and-Whisker plot of Inverted Microscope trials of $\mathrm{NaOH}$ and $\mathrm{MC}$ treatment at $25 \mathrm{~V} / \mathrm{cm}$

Summary Statistics
\begin{tabular}{|l|l|l|l|l|l|}
\hline & $D-$ Trial1 & D- Trial2 & D- Trial 3 & D- Trial4 & D- Trial5 \\
\hline Count & 113 & 221 & 705 & 262 & 201 \\
\hline Average & 59.634 & 2577.71 & 109.899 & 1734.32 & 76.304 \\
\hline Standard deviation & 0.488751 & 794.849 & 23.0862 & 28.2377 & 16.0201 \\
\hline Coeff. of variation & $0.819585 \%$ & $30.8355 \%$ & $21.0068 \%$ & $1.62817 \%$ & $20.9951 \%$ \\
\hline Minimum & 58.796 & 1326.47 & 67.708 & 1634.21 & 60.906 \\
\hline Maximum & 60.653 & 3833.82 & 178.964 & 1781.2 & 117.829 \\
\hline Range & 1.857 & 2507.35 & 111.256 & 146.985 & 56.923 \\
\hline Stnd. skewness & 1.83855 & -0.965052 & 8.5838 & -9.80755 & 6.92848 \\
\hline Stnd. kurtosis & -1.95734 & -4.13112 & 8.49632 & 5.89104 & 1.4125 \\
\hline
\end{tabular}

The StatAdvisor

This table shows summary statistics for each of the selected data variables. It includes measures of central tendency, measures of variability, and measures of shape. Of particular interest here are the standardized skewness and standardized kurtosis, which can be used to determine whether the sample comes from a normal distribution. Values of these statistics outside the range of -2 to +2 indicate significant departures from normality, which would tend to invalidate many of the statistical procedures normally applied to this data. In this case, the following variables show standardized skewness values outside the expected range:
D- Trial 3
D- Trial4
D- Trial5

The following variables show standardized kurtosis values outside the expected range:

D- Trial2

D- Trial 3

D- Trial4

To make the variables more normal, you might try a transformation such as LOG(Y), SQRT(Y), or 1/Y.

Figure 59: Data summary of all test runs with $\mathrm{NaOH}$ and $\mathrm{MC}$ treatment at $25 \mathrm{~V} / \mathrm{cm}$ 